COMMUNICATIONS IN

ANALYSIS AND GEOMETRY

Volume 11, Number 3, 489-551, 2003

\title{
A New 3-dimensional Curvature Integral Formula for PL-manifolds of Non-positive Curvature
}

\author{
Jianguo CAO And José F. Escobar
}

\section{Introduction.}

In this paper, we derive a new curvature integral formula for 3-dimensional piecewise linear manifolds with singularities. Among other things, we also present a sharp isoperimetric inequality for 3-dimensional PL-manifolds of non-positive curvature by using this new curvature integral formula.

Let $\Omega$ be a smooth compact domain in a smooth Riemannian manifold, and $G K_{\partial \Omega}$ represent the Gauss-Kronecker curvature (i.e., the determinant of the second fundamental form) of the boundary of $\Omega, \partial \Omega$. A well-known Theorem of Chern-Lashof [CL] states that for any compact convex smooth domain $\Omega$ in $\mathbb{R}^{n}$, the total Gauss-Kronecker curvature of its boundary satisfies

$$
\int_{\partial \Omega} G K_{\partial \Omega} d A=\operatorname{vol}_{n-1}\left(S^{n-1}\right)
$$

where $S^{n-1}$ is the unit $(n-1)$-dimensional sphere in the $n$-dimensional Euclidean space. It has been conjectured by various authors that for any compact convex smooth domain $\Omega$ in a Cartan-Hadamard manifold $M^{n}$, the total Gauss-Kronecker curvature of its boundary satisfies

$$
\int_{\partial \Omega} G K_{\partial \Omega} d A \geq \operatorname{vol}_{n-1}\left(S^{n-1}\right)
$$

In fact, for a compact surface $\Sigma$ in a 3-dimensional smooth Cartan-Hadamard manifold $M^{3}$, the classical Gauss Theorem states

$$
K_{\Sigma}-\left.K_{M^{3}}\right|_{\Sigma}=G K_{\Sigma}
$$

where $K_{\Sigma}$ (resp. $K_{M^{3}}$ ) is the sectional curvature of $\Sigma\left(\operatorname{resp} . M^{3}\right)$. It follows from the equation (0.2) and the Gauss-Bonnet formula that if $\partial \Omega$ is

\footnotetext{
${ }^{1}$ Both authors were supported in part by NSF grants.
} 
an embedded smooth sphere in a 3-dimensional smooth Cartan-Hadamard manifold $M^{3}$ then

$$
\int_{\partial \Omega} G K_{\partial \Omega} d A \geq 4 \pi
$$

see $[K]$.

In this paper, we consider non-smooth 3-dimensional manifolds allowing the possibility of singularities. Through out this paper $X^{n}$ stands for an $n$-dimensional simply connected piecewise linear manifold with non-positive curvature. The curvature we consider here is the one defined by using comparison triangles. In our case these manifolds are part of the family of $C A T(0)$ spaces (see $[\mathrm{BH}]$ ). In fact, if the sum of interior angles of any geodesic triangles in $X^{n}$ is less than or equal to $\pi$, then $X^{n}$ has non-positive curvature. For any piecewise smooth convex domain $\Omega$ in $\mathbb{R}^{n}$, Federer [Fe1], introduced curvature measures associated to $\Omega$ by using the coefficients of the so-called Steiner polynomial of $\Omega$. We introduce the outer Gauss-Kronecker curvature measure $G K_{\partial \Omega}$ for convex domains $\Omega$ in a PL-manifold $X^{n}$ in a similar way, see (2.1) below and prove a new curvature integral formula for some domains in a piecewise linear manifold with non-positive curvature.

Main Theorem. Let $X^{3}$ be a 3-dimensional simply connected piecewise linear manifold with non-positive curvature. If $\Omega$ is a compact convex domain with non-empty interior, then its total Gauss-Kronecker curvature measure is given by the following formula:

$$
\begin{aligned}
& \int_{\partial \Omega} d\left(G K_{\partial \Omega}\right)=4 \pi+ \\
& \sum_{p \in(\partial \Omega)} \sum_{\sigma^{1} \subset S t(p)} \sum_{v \in \operatorname{Link}\left(p, \sigma^{1}\right)}\left[\left|\operatorname{Link}\left(\sigma^{1}, X^{3}\right)\right|-2 \pi\right] \sin \left[\theta_{p}^{*}(v, \Omega)\right] .
\end{aligned}
$$

where $\theta_{p}^{*}(v, \Omega)=\min \left\{\theta_{p}(v, \Omega), \frac{\pi}{2}\right\}, \theta_{p}(v, \Omega)$ is the angle between the vector $v$ and the tangent cone $T_{p}(\Omega)$ of $\Omega$ at the point $p \in \partial \Omega$, and $\operatorname{Link}\left(\sigma^{1}, X^{3}\right)$ denotes the set of unit vectors orthogonal to the simplex $\sigma^{1}$.

It is known that if $X^{3}$ has non-positive curvature the length of Link $\left(\sigma^{1}, X^{3}\right)$ is greater or equal to $2 \pi$. Hence, the last summation term in formula (0.4) is always non-negative. Ballmann and Buyalo [BB] proved a Gauss-Bonnet type formula for piecewise smooth metrics on 2-polyhedra with a local group action. Our result applies to 3-dimensional domain which do not necessarily admit co-compact group actions.

The main point of this paper is to understand how singularities are related to the total integral of the Gauss-Kronecker curvature. In order to 
do that we first show that $\operatorname{Sing}\left(X^{n}\right)$ is a closed, piecewise linear subset of codimension 2 in $X^{n}$. In fact, we prove that $\operatorname{Sing}\left(X^{n}\right)=\bigcup_{i} \sigma_{i}^{n-2}$. where $\sigma_{i}^{n-2}$ is an $(n-2)$-dimensional simplex.

To prove the above formula we first estimate the total outer GaussKronecker curvature of a convex piecewise linear domain and then show that the outer Gauss-Kronecker curvature measure of a sequence of convex domains converging to a convex domains in the Hausdorff topology is upper semi-continuous.

One of the main ingredients in the proof of the Main Theorem is the detailed analysis on the equidistance hypersurface $\partial \Omega_{s}$ where $\Omega_{s}=\{x \in$ $\left.X^{n} \mid d(x, \Omega)<s\right\}$. We show that $\left[\partial \Omega_{s}-\operatorname{Sing}\left(X^{n}\right)\right]$ is a $C^{1,1}$ hypersurface, whenever $\Omega$ is convex and $s>0$. When $n=3, \partial \Omega_{s}$ is a surface with possible singularities. A version of Gauss-Bonnet formula is applicable to the surface $\partial \Omega_{s}$, which yields formula (0.4) for $\Omega_{s}$ and letting $s \rightarrow 0$, we derive the curvature integral formula (0.4) for $\Omega$.

The main application of our integral formula is the derivation of a sharp isoperimetric inequality in 3-dimensional PL-manifolds of non-positive curvature.

Main Corollary. Let $X^{3}$ be a 3-dimensional simply connected piecewise linear manifold with non-positive curvature. If $\Omega$ is a compact piecewise smooth domain, then

$$
\operatorname{vol}(\Omega) \leq \frac{1}{6 \sqrt{\pi}}[\operatorname{Area}(\partial \Omega)]^{\frac{3}{2}} .
$$

Equality holds if and only if $\Omega$ is isometric to the ball in the Euclidean space $\mathbb{R}^{3}$.

The last inequality, was proved by Kleiner $[\mathrm{K}]$ in the context of 3dimensional simply connected smooth Riemannian manifolds with nonpositive sectional curvature. Similar results were obtained in the 2 and 4 dimensional cases by Weil [W] and Croke [Cr1] respectively.

The authors would like to thank the referee for many helpful comments and questions that made this final version a more readable one.

\section{Preliminary results.}

Throughout this paper, all k-simplexes are always assumed to be open, each $\mathrm{k}$-simplex is isometric to an open set in $\mathbb{R}^{k}$.

In this section we recall some preliminary results of piecewise smooth manifolds with curvature bounded above. There results are needed for later sections. 


\section{1.a. The orthogonal join and a volume comparison theorem.}

In our paper, we need to study the tangent cone $T_{p}\left(X^{n}\right)$ of a piecewise linear manifold $X^{n}$ at any given point $p \in X^{n}$. The unit tangent cone is the subset of all unit vector in $T_{p}\left(X^{n}\right)$, which is denoted by $\operatorname{Link}\left(p, X^{n}\right)$. The local geometry of $X^{n}$ is related to $\operatorname{Link}\left(p, X^{n}\right)$. If $X_{1}$ and $X_{2}$ are two piecewise linear manifolds, then we would like to recall some elementary facts about the relations among $\operatorname{Link}\left(p_{1}, X_{1}\right), \operatorname{Link}\left(p_{2}, X_{2}\right)$ and $\operatorname{Link}\left(\left(p_{1}, p_{2}\right), X_{1} \times X_{2}\right)$.

For this purpose, we first recall the definition the orthogonal join $L_{1} * L_{2}$ of two piecewise spherical polyhedra $L_{1}$ and $L_{2}$. It is a piecewise spherical polyhedron of dimensional equal to $\operatorname{dim} L_{1}+\operatorname{dim} L_{2}+1$.

Definition 1.0. Suppose that $\sigma^{i} \subset L_{1}$ is a spherical $i$-cell and that $\sigma^{j} \subset L_{2}$ is a spherical $j$-cell. Locally, we identify $\sigma^{i}$ and $\sigma^{j}$ with subsets in the unit spheres $S^{i}$ and $S^{j}$. Furthermore, we identify $S^{i}$ and $S^{j}$ with the unit spheres in subspaces of $\mathbb{R}^{i+j+2}$ which are orthogonal complements. Then $\sigma^{i} * \sigma^{j}$, the orthogonal join of $\sigma^{i}$ and $\sigma^{j}$, is the $(i+j+1)$-cell in $S^{i+j+1}$ defined as the convex hull of $\sigma^{i}$ and $\sigma^{j}$ in $S^{i+j+1}$ (i.e., $\sigma^{i} * \sigma^{j}$ is the union of all geodesic segments in $S^{i+j+1}$ which begin in $\sigma^{i}$ and end in $\sigma^{j}$ ). More precisely,

$$
\sigma^{i} * \sigma^{j}=\left\{(\cos \theta) v+(\sin \theta) w \mid 0 \leq \theta \leq \frac{\pi}{2}, v \in \sigma^{i}, w \in \sigma^{j}\right\} .
$$

In an obvious fashion, we can glue all these $\left\{\sigma^{i} * \sigma^{j}\right\}$ together to obtain $L_{1} * L_{2}$.

In particular, if $L_{1}$ is the round sphere $S^{k-1}$, then $S^{k-1} * L_{2}$ is called the $k$-fold suspension of $L_{2}$.

It is straightforward to see that if $X_{1}$ and $X_{2}$ be two piecewise linear manifolds, then $\operatorname{Link}\left(\left(p_{1}, p_{2}\right), X_{1} \times X_{2}\right)=\left[\operatorname{Link}\left(p_{1}, X_{1}\right)\right] *\left[\operatorname{Link}\left(p_{2}, X_{2}\right)\right]$. Using this fact and an induction method on dimensions, we will prove a volume comparison theorem for piecewise spherical manifolds.

By the well-known comparison theorem of Aleksandrov and Topogonov, the statement that sectional curvature of a smooth Riemannian manifold $M$ is bounded above by a real number $c$ is equivalent to a statement concerning small geodesic triangles in $M$. One such statement, the so-called "CAT(c)" inequality, compares distances between points in a triangle with the corresponding distances in a comparison triangle in the complete, simply connected, 2-manifold of constant curvature $c$ (for a precise definition see $[\mathrm{ChD}]$ p932 or $[\mathrm{BH}])$. Here we say that a geodesically complete space $L$ has curvature $\leq 1$ if the $C A T(1)$-inequality holds for small geodesic triangles in $L$; and we say that the space $L$ satisfies the $C A T(1)$ inequality (or $L$ is called a $C A T(1)$ space) if the $C A T(1)$ inequality holds for all geodesic triangles. 
The following result of $[\mathrm{ChD}]$ will be used frequently in this paper.

Lemma 1.1. [ChD, p1002], Let $L^{m}$ be a piecewise spherical manifold satisfying $C A T(1)$ inequality, $v_{i} \in S^{k}, w_{i} \in L^{m}$ and $0 \leq t_{i} \leq \frac{\pi}{2}$ for $i=1,2$. Suppose that $\xi_{i}=\left[\left(\cos t_{i}\right) v_{i}+\left(\sin t_{i}\right) w_{i}\right] \in\left[S^{k} * L\right]$ for $i=1,2$. Then $d_{S^{k} * L}\left(\xi_{1}, \xi_{2}\right) \leq \pi$. If $d_{S^{k} * L}\left(\xi_{1}, \xi_{2}\right)=\pi$ then one of the following holds:

(1) $t_{1}=t_{2}=0$ and $v_{1}=-v_{2} \in S^{k}$;

(2) $t_{1}=t_{2}=\frac{\pi}{2}$ and $d_{L}\left(w_{1}, w_{2}\right) \geq \pi$;

(3) $0<t_{1}=t_{2}<\frac{\pi}{2}, v_{1}=-v_{2} \in S^{k}$ and $d_{L}\left(w_{1}, w_{2}\right) \geq \pi$.

We will prove a volume comparison theorem for spherical singular $C A T(1)$ spaces. This is a particular case of our main estimate in this paper. More precisely we show the following theorem.

Theorem 1.2. Let $L^{n-1}$ be a piecewise spherical manifold of dimension $(n-1)$ satisfying the CAT(1) inequality. Then

$$
\operatorname{vol}_{n-1}\left(L^{n-1}\right) \geq \operatorname{vol}_{n-1}\left(S^{n-1}(1)\right) .
$$

Equality holds if and only if $L^{n-1}$ is isometric to $S^{n-1}(1)$.

If $X^{n}$ is a piecewise Euclidean $P L$-manifold of non-positive curvature then for every $x \in X^{n}$, the unit tangent cone $\operatorname{Link}\left(x, X^{n}\right)$ of $X$ at $x$ is a piecewise spherical manifold satisfying the $C A T(1)$ inequality. Therefore we obtain

$$
\operatorname{vol}_{n-1}\left[\operatorname{Link}\left(x, X^{n}\right)\right] \geq \operatorname{vol}_{n-1}\left(S^{n-1}(1)\right) .
$$

Furthermore, equality holds if and only if $\operatorname{Link}\left(x, X^{n}\right)$ is isometric to $S^{n-1}(1)$.

In order to prove the above theorem we first need some new notations.

Definition 1.3. Let $Y$ be a polyhedron of piecewise constant curvature. A path $\sigma:[a, b] \rightarrow Y$ is a broken geodesic path if there exist numbers $t_{0}, \ldots, t_{k}$ with $a=t_{0}<t_{1}<\cdots<t_{k}=b$ so that for each $i, 0 \leq i<k,\left.\sigma\right|_{\left[t_{i}, t_{i+1}\right]}$ is a geodesic path with image lying entirely in some closed cell of $Y$. By a broken geodesic we shall mean the image of broken geodesic path together with an orientation. If a broken geodesic is the image of a geodesic path (that is, a path locally isometric to an interval) then it is called a local geodesic.

Suppose that $y_{0}, y_{1}$ are two points in some closed $m$-simplex $\bar{\sigma}^{m}$ of $Y$ and $\varphi$ is a geodesic segment in $\bar{\sigma}^{m}$ from $y_{0}$ to $y_{1}$. Then $\varphi$ determines a unit tangent vector in $T_{y_{0}}\left(\bar{\sigma}^{m}\right)$ and hence, a point in $\operatorname{Link}\left(y_{0}, Y\right)$ called the 
outgoing tangent vector of $\varphi$ in $y_{0}$ and denoted by $\varphi_{\text {out }}^{\prime}\left(y_{0}\right)$. Similarly, $\varphi$ determines an incoming tangent vector $\varphi_{i n}^{\prime}(y) \in \operatorname{Link}(y, Y)$.

Suppose that for $1 \leq i \leq k, \varphi_{i}$ is a geodesic segment in some cell $\bar{\sigma}^{m}$ of $Y$ from $y_{i-1}$ to $y_{i}$. The $\varphi_{i}$ 's can be glued together to give a broken geodesic $\varphi$ from $y_{0}$ to $y_{k}$. We shall use the notation $\varphi=\left(\varphi_{1}, \ldots, \varphi_{k}\right)$. The incoming and outgoing vectors of the broken geodesic $\varphi$ make an "angle" $\theta_{i}$ at each point $y_{i}$, defined by,

$$
\theta_{i}=d\left(\left(\varphi_{i}\right)_{\text {in }}^{\prime}\left(y_{i}\right),\left(\varphi_{i+1}\right)_{\text {out }}^{\prime}\left(y_{i}\right)\right)
$$

where $d$ denotes distance in $\operatorname{Link}\left(y_{i}, Y\right)$.

The local characterization of a geodesic can be expressed in terms of angles $\left\{\theta_{i}\right\}_{i=1}^{m}$. The following lemma is well known.

Lemma 1.4. With notation as above, the broken geodesic $\varphi=\left(\varphi_{1}, \ldots, \varphi_{m}\right)$ is a local geodesic if and only if $\theta_{i} \geq \pi$ for $1 \leq i \leq m$.

To prove the Theorem 1.2 we first extend the classical Bishop Comparison Theorem for Riemannian manifolds to spherical singular spaces.

Theorem 1.5. Suppose that $L^{n-1}$ is a piecewise spherical manifold which satisfies the CAT(1) inequality. For any point $p \in L$ and $0<r<\pi$, let $B_{r}(p)=\left\{q \in L^{n-1} \mid d_{L}(p, q) \leq r\right\}$. Then

$$
\operatorname{vol}_{n-1}\left(B_{r}(p)\right) \geq \operatorname{vol}_{n-1}\left(\hat{B}_{r}(\hat{p})\right),
$$

where $\hat{B}_{r}(\hat{p})=\left\{\hat{q} \in S^{n-1}(1) \mid d(\hat{q}, \hat{p}) \leq r\right\}$.

The proof of Theorem 1.5 uses a variant of Toponogov's comparison theorem. In fact, the following Proposition 1.6 and its counterpart provide an equivalent definition of $C A T(1)$ space, (see $[\mathrm{ABN}]$ ). The following two results are well known, (e.g., cf. [BH, p161-162]).

Proposition 1.6. Let $L$ be as in Theorem 1.5. Suppose that $\left\{v_{1}, v_{2}, v_{3}\right\}$ are three points in $L$. Let $\Delta$ be a geodesic triangle of perimeter $\leq 2 \pi$ in $L$ with vertices $\left\{v_{1}, v_{2}, v_{3}\right\}$ and $\alpha_{i}$ the interior angle at $v_{i}, i=1,2,3$. If $\hat{\Delta}$ is a comparison triangle of the same edge length in $S^{2}(1), \hat{\alpha}_{i}, i=1,2,3$ are corresponding interior angles of $\hat{\Delta}$, then $\alpha_{i} \leq \hat{\alpha}_{i}$, as long as $d_{L}\left\{v_{i}, v_{j}\right\}<\pi$, $i, j=1,2,3$.

Proposition 1.7. Let $L$ be a piecewise spherical manifold satisfying the $C A T(1)$ inequality. Assume that $\left(\varphi_{1}, \varphi_{2}, \alpha\right)$ is a geodesic hinge of perimeter $\leq 2 \pi$ in $L$, where $\varphi_{1}$ and $\varphi_{2}$ are length-minimizing geodesic segments of length $<\pi$ with $\varphi_{1}\left(\ell_{1}\right)=\varphi_{2}(0)$. If $\left(\hat{\varphi}_{1}, \hat{\varphi}_{2}, \alpha\right)$ is a corresponding geodesic 
hinge in $S^{2}(1)$ such that $\ell_{i}=\ell\left(\hat{\varphi}_{i}\right)=\ell\left(\varphi_{i}\right)$, for $i=1,2$, and $0<\alpha<\pi$, then

$$
\ell_{3}=d_{L}\left(\varphi_{2}\left(\ell_{2}\right), \varphi_{1}(0)\right) \geq d_{S^{2}(1)}\left(\hat{\varphi}_{2}\left(\ell_{2}\right), \hat{\varphi}_{1}(0)\right)=\hat{\ell}_{3}
$$

Proof of Theorem 1.5. We first assume that $p$ is a regular point, i.e., $T_{p}\left(L^{n-1}\right)$ is isometric to $R^{n-1}$. Because $L$ satisfies the CAT(1) inequality, any pair of points in $L$ of distance $<\pi$ can be connected by a unique length-minimizing geodesic (cf. [ChD, p.933]). Therefore, for $0<r<\pi$ and $q \in \partial B_{r}(p)$, there is a unique geodesic $\varphi_{p q}:[0, r] \rightarrow L$ from $p$ to $q$. Let $\{(t, \theta)\}$ be the geodesic polar normal coordinate system of $S^{n-1}(1)$ around the point $\hat{p}$. We define a Lipschitz map

$$
\begin{aligned}
F: B_{r}(p) & \longrightarrow \hat{B}_{r}(\hat{p}) \\
\varphi_{p q}(t) & \longrightarrow\left(t, \varphi_{p q}^{\prime}(0)\right) .
\end{aligned}
$$

It follows from Proposition 1.7 that the Lipschitz constant of $F$ is $\leq$ 1. Thus $F$ is a distance non-increasing map. To verify that $F$ is onto observe that given two points in $L$ with distance less than $\pi$ there is a unique minimizing geodesic joining them. This is because $L$ satisfies the CAT(1) inequality. Hence any length minimizing geodesic with length strictly less than $\pi$ can be extended to a longer length minimizing geodesic. Thus, we conclude that $F$ is onto and hence

$$
\operatorname{vol}_{n-1}\left(B_{r}(p)\right) \geq \operatorname{vol}_{n-1}\left(\hat{B}_{r}(\hat{p})\right) .
$$

For general case, we observe that the set of regular points is a dense subset of $L^{n-1}$. Taking the limit in above inequality, one completes the proof.

Theorem 1.5 can be strengthened as follows:

Theorem 1.8. Let $L^{n-1}$ be a piecewise spherical manifold of dimension $(n-1)$ satisfying CAT(1) inequality, $p \in L^{n-1}$ and $L_{p}^{n-2}=\operatorname{Link}\left(p, L^{n-1}\right)$. Suppose that $\hat{L}_{p}=S^{0} * L_{p}^{n-2}$ is the two point suspension of $L_{p}^{n-2}, \hat{p} \in S^{0}$ and that $\hat{B}_{r}(\hat{p})=\left\{q \in \hat{L}_{p} \mid d(\hat{p}, q)<r\right\}$. Then

(1) $\hat{L}_{p}$ satisfies the CAT(1) inequality,

(2) $\operatorname{vol}_{n-1}\left(\hat{B}_{r}(\hat{p})\right)=\operatorname{vol}_{n-2}\left(L_{p}^{n-2}\right) \int_{0}^{r}(\sin t)^{n-2} d t$,

(3) $\operatorname{vol}_{n-1}\left(B_{r}(p)\right) \geq \operatorname{vol}_{n-1}\left(\hat{B}_{r}(\hat{p})\right)$, where $B_{r}(p)=\left\{q \in L^{n-1} \mid d(p, q)<\right.$ $r$ \} and $0<r<\pi$. 
Proof. (1) Let $X^{n-1}=\mathcal{C}\left(L_{p}^{n-2}\right)$ be the cone over $L_{p}^{n-2}$. Observe that $L_{p}^{n-2}$ satisfies the CAT(1) inequality because $X^{n-1}$ satisfies the CAT(0) inequality (cf. [Bri]). Let $Y^{n}=\mathbb{R} \times X^{n-1}$. Clearly, $Y$ satisfies the CAT(0) inequality. It follows that $\hat{L}_{p}=S^{0} * \operatorname{Link}\left(0, X^{n-1}\right)=\operatorname{Link}(0, Y)$ satisfies the $\operatorname{CAT}(1)$ inequality.

(2) Let $d w^{2}$ be the piecewise spherical metric on $L_{p}^{n-2}$. Then the metric $d s^{2}$ of $\hat{L}_{p}$ has a wrapped product structure

$$
d s^{2}=d t^{2}+(\sin t)^{2} d w^{2} .
$$

Therefore, using the wrapped product structure, we have

$$
\begin{aligned}
\operatorname{vol}_{n-1}\left(\hat{B}_{r}(\hat{p})\right) & =\int_{0}^{r} \int_{w \in L_{p}^{n-2}}(\sin t)^{n-2} d w d t \\
& =\operatorname{vol}_{n-2}\left(L_{p}^{n-2}\right) \int_{0}^{r}(\sin t)^{n-2} d t .
\end{aligned}
$$

(3) We proceed as in the proof of Theorem 1.5 and omit the details here. Proof of Theorem 1.2. Letting $r \rightarrow \pi$ in Theorem 1.5, we have

$$
\operatorname{vol}_{n-1}\left(L^{n-1}\right) \geq \operatorname{vol}_{n-1}\left(S^{n-1}(1)\right) .
$$

In what follows, we are going to show that if $L^{n-1}$ is a piecewise spherical manifold of dimension $(n-1)$ satisfying the CAT(1) inequality and if $\operatorname{vol}_{n-1}\left(L^{n-1}\right)=\operatorname{vol}_{n-1}\left(S^{n-1}(1)\right)$ then $L^{n-1}$ is isometric to the unit sphere $S^{n-1}(1)$. The proof of this assertion will use an induction method on the dimension of $L^{n-1}$ and $k$-fold suspension of piecewise spherical spaces. We will first show that $L^{n-1}$ has no singularities when the equality holds. The definition of singularity is given by Definition 1.10 below.

When $n-1=2$ if Area $\left(L^{2}\right)=4 \pi$, we claim that $L^{2}$ is isometric to $S^{2}(1)$. Suppose that $L^{2}$ is not isometric to $S^{2}(1)$. Then there exists a singular point $p \in L^{2}$ such that $\left|\operatorname{Link}\left(p, L^{2}\right)\right|>2 \pi$. Let $\hat{L}_{p}=S^{0} * \operatorname{Link}\left(p, L^{2}\right)$. Using Theorem 1.8, we have

$$
\operatorname{Area}\left(L^{2}\right) \geq \operatorname{Area}\left(\hat{L}_{p}\right)=\left|\operatorname{Link}\left(p, L^{2}\right)\right| \int_{0}^{\pi} \sin t d t=2\left|\operatorname{Link}\left(p, L^{2}\right)\right|>4 \pi
$$

which contradicts to Area $\left(L^{2}\right)=4 \pi$.

Let us now suppose that Theorem 1.2 is true for dimension $(n-2)$. The inequality (1.3) follows from the first part of Theorem 1.2. When 
$\operatorname{vol}_{n-1}\left(L^{n-1}\right)=\operatorname{vol}_{n-1}\left(S^{n-1}(1)\right)$ we claim that $L^{n-1}$ must be isometric to $S^{n-1}(1)$. Otherwise, there is a singular point $p \in L^{n-1}$ such that $L_{p}^{n-2}=\operatorname{Link}\left(p, L^{n-1}\right)$ is not isometric to $S^{n-2}(1)$. Since $L^{n-1}$ satisfies the CAT(1) inequality so does $L_{p}^{n-2}$ (cf. $[\mathrm{ChD}]$ ). By induction, we know that

$$
\operatorname{vol}_{n-2}\left(L_{p}^{n-2}\right)>\operatorname{vol}_{n-2}\left(S^{n-2}(1)\right) .
$$

Let us now consider $\hat{L}_{p}=S^{0} * L_{p}^{n-2}$. It follows from Theorem 1.8 that

$$
\begin{aligned}
\operatorname{vol}_{n-1}(L) & \geq \operatorname{vol}_{n-1}\left(B_{\pi}(p)\right) \geq \operatorname{vol}_{n-1}\left(B_{\pi}^{*}\left(p^{*}\right)\right)=\operatorname{vol}_{n-1}\left(\hat{L}_{p}\right) \\
& =\operatorname{vol}_{n-2}\left(L_{p}^{n-2}\right) \int_{0}^{\pi}(\sin t)^{n-2} d t .
\end{aligned}
$$

Using (1.4)-(1.5), we get $\operatorname{vol}_{n-1}(L)>\operatorname{vol}_{n-1}\left(S^{n-1}(1)\right)$ which is a contradiction. Hence there is no singular points on $L$, and $L$ is a smooth Riemannian manifold of constant sectional curvature 1 . Thus, $L$ is a quotient space of $S^{n-1}(1)$ with the induced metric. Therefore $\operatorname{vol}_{n-1}(L) \leq \operatorname{vol}_{n-1}\left(S^{n-1}(1)\right)$, equality holds if and only if $L$ is isometric to $S^{n-1}(1)$.

\section{curvature. \\ 1.b. The singular set of PL-manifolds with non-positive}

In this sub-section we first discuss some properties of the singular set of the manifold $X^{n}$.

Definition 1.9. Given linear simplexes $\sigma^{k} \subset \sigma^{n}$, at any point $q \in \sigma^{k}$, the normal cone $\mathcal{C}^{\perp}\left(\sigma^{k}, \sigma^{n}\right)$ is the set consisting of all rays through $q$ which are orthogonal to $\sigma^{k}$ and point into $\sigma^{n}$. The associated spherical simplex, Link $\left(\sigma^{k}, \sigma^{n}\right)$ is called the link of $\sigma^{k}$ in $\sigma^{n}$.

Using the definition above we give a description of a singular simplex in a PL-manifold.

Definition 1.10. Let $\tau$ be a triangulation of $X^{n}$.

(1) If $\sigma_{0}^{k}$ is a $k$-simplex of $X^{n}$, then

$$
\operatorname{Link}\left(\sigma_{0}^{k}, X^{n}\right)=\bigcup_{\sigma^{n} \supset \sigma_{0}^{k}} \overline{\operatorname{Link}\left(\sigma_{0}^{k}, \sigma^{n}\right)}
$$

where $\operatorname{Link}\left(\sigma^{k}, \sigma^{n}\right)$ is given by Definition 1.9.

(2) A vertex $\sigma^{0} \in X^{n}$ is said to be singular if Link $\left(\sigma^{0}, X^{n}\right)$ is not isometric to the unit $(n-1)$-sphere $S^{n-1}(1)$. Equivalently, $x \in X^{n}$ is singular 
if $T_{x}\left(X^{n}\right)$ is not isometric to $\mathbb{R}^{n}$. A $k$-dimensional simplex $\sigma^{k} \subset X^{n}$ is said to be singular if Link $\left(\sigma^{k}, X^{n}\right)$ is not isometric to the unit sphere $S^{n-k-1}(1)$.

(3) Suppose that $L$ is a subset of $\operatorname{Link}\left(x, X^{n}\right)$. Then the dual link $L^{*}$ given by all unit vectors in $\operatorname{Link}\left(x, X^{n}\right)$ making an angle $\geq \frac{\pi}{2}$ with every vector of $L$, where by the angle $\varangle(v, w)$ we mean the distance between two unit vector $v$ and $w$ in $\operatorname{Link}\left(x, X^{n}\right)$.

For a PL-manifold we have the following basic observation about the singular set.

Proposition 1.11. The set $\operatorname{Sing}\left(X^{n}\right)$ is closed and $\operatorname{dim}\left[\operatorname{Sing}\left(X^{n}\right)\right] \leq n-2$.

Proof. If $\sigma^{n-1}$ is a $(n-1)$-simplex of $X^{n}$, then $\operatorname{dim}\left[\operatorname{Link}\left(\sigma^{n-1}, X^{n}\right)\right]=$ 0 . Because $X^{n}$ is a PL-manifold without boundary, each $\sigma^{n-1}$ must be a common face of exactly two $n$-simplexes. Since the $n$-simplexes are glued along totally geodesic boundaries, each open $\sigma^{n-1}$ is regular; it follows that $\operatorname{dim}\left[\operatorname{Sing}\left(X^{n}\right)\right] \leq n-2$. It is easy to check that for any PL-manifold, the singular set is closed.

In the next proposition we use Theorem 1.2 to show the non-existence of isolated singularities in a PL-manifold $X^{n}$ of non-positive curvature and dimension greater or equal to three.

Proposition 1.12. The manifold $X^{n}$ of non-positive curvature has no isolated singularities when $n \geq 3$.

Proof. Let $x \in X^{n}$ be an isolated singularity. Then Link $\left(x, X^{n}\right)$ is a smooth manifold of constant curvature 1 . Moreover $L^{n-1}=\operatorname{Link}\left(x, X^{n}\right)$ is a space form. When $n-1 \geq 2$, any smooth space form $L^{n-1}$ of constant curvature 1 is covered by the unit sphere $S^{n-1}(1)$. Therefore

$$
\operatorname{vol}_{n-1}\left(\operatorname{Link}\left(x, X^{n}\right)\right) \leq \operatorname{vol}_{n-1}\left(S^{n-1}(1)\right)
$$

when $n-1 \geq 2$. By Theorem 1.2, inequality (1.1) holds and then

$$
\operatorname{vol}_{n-1}\left(\operatorname{Link}\left(x, X^{n}\right)\right)=\operatorname{vol}_{n-1}\left(S^{n-1}(1)\right)
$$

and $\operatorname{Link}\left(x, X^{n}\right)$ is isometric to $S^{n-1}(1)$, which is a contradiction to the assumption that $x$ is a singularity.

We remark that Proposition 1.12 is not true without the assumption of non-positive curvature. For example, let $Y^{n}$ be a cone over $S^{n-1}$ in $R^{n+1}$ with a base point $y_{0}$. Such a space $Y^{n}$ has positive curvature at the isolated singular point $y_{0}$. 
In the next proposition we identify the structure of the singular set of the space $X^{n}$.

Proposition 1.13. Suppose that $\sigma^{k} \subset \operatorname{Sing}\left(X^{n}\right)$ is a $k$-dimensional simplex contained in $\operatorname{Sing}\left(X^{n}\right)$ with $k<n-2$. Then there exists an $(n-2)-$ dimensional simplex $\sigma^{n-2} \subset \operatorname{Sing}\left(X^{n}\right)$ such that $\sigma^{k} \subset \partial \sigma^{n-2}$. Hence the singular set is a union of simplices of dimension $(n-2)$.

Proof. Because Proposition 1.12 we can assume that $k \geq 1$. For each $q \in \operatorname{Int}\left(\sigma^{k}\right)$, there is a neighborhood of $q$ in the form of

$$
U^{k} \times \mathcal{C}_{\varepsilon}\left(\operatorname{Link}\left(\sigma^{k}, X^{n}\right)\right),
$$

where $q \in U^{k} \subset \sigma^{k}$ and $\mathcal{C}_{\varepsilon}\left(\operatorname{Link}\left(\sigma^{k}, X^{n}\right)\right)$ is the set of points in the normal cone at $q \in \sigma^{k}$ having distance to the vertex $q$ less than $\varepsilon,(\operatorname{cf}[\mathrm{CMS}])$. By the assumption we have $\operatorname{dim}\left(\operatorname{Link}\left(\sigma^{k}, X^{n}\right)\right)=n-k-1 \geq 2$. Since $\sigma^{k} \subset \operatorname{Sing}\left(X^{n}\right), \operatorname{Link}\left(\sigma^{k}, X^{n}\right)$ is not isometric to $S^{n-k-1}(1)$. Because $X^{n}$ satisfies the $\operatorname{CAT}(0)$ inequality, $\operatorname{Link}\left(\sigma^{k}, X^{n}\right)$ satisfies the CAT(1) inequality. Theorem 1.12 implies that

$$
\operatorname{vol}_{n-k-1}\left(\operatorname{Link}\left(\sigma^{k}, X^{n}\right)\right)>\operatorname{vol}_{n-k-1}\left(S^{n-k-1}(1)\right) .
$$

Therefore Link $\left(\sigma^{k}, X^{n}\right)$ cannot be a smooth Riemannian manifold of constant sectional curvature 1 . Otherwise, $\operatorname{Link}\left(\sigma^{k}, X^{n}\right)$ would be covered by $S^{n-k-1}(1)$ and $\operatorname{vol}_{n-k-1} \operatorname{Link}\left(\sigma^{k}, X^{n}\right) \leq \operatorname{vol}_{n-k-1}\left(S^{n-k-1}(1)\right)$. Hence, there must be a vector $v_{k+1} \in \operatorname{Link}\left(\sigma^{k}, X^{n}\right)$ such that $T_{v_{k+1}}\left(\operatorname{Link}\left(\sigma^{k}, X^{n}\right)\right)$ is not isometric to $\mathbb{R}^{n-k-1}$. Therefore, $\operatorname{Link}\left(v_{k+1}, \operatorname{Link}\left(\sigma^{k}, X^{n}\right)\right)$ is not isometric to $S^{n-k-2}$. Let us choose small positive number $\delta$ and set $q_{k+1}=\operatorname{Exp}_{q}\left(\delta v_{k+1}\right)$. Using the above decomposition, we see that there is a $(k+1)$-simplex $\sigma^{k+1}$ containing both $\sigma^{k}$ and $q_{k+1}$ and $\sigma^{k} \subset \partial \sigma^{k+1}$. It is easy to see that $\operatorname{Link}\left(\sigma^{k+1}, X^{n}\right)$ is isometric to $\operatorname{Link}\left(v_{k+1}, L\left(\sigma^{k}, X^{n}\right)\right)$. Thus Link $\left(\sigma^{k+1}, X^{n}\right)$ is not isometric to $S^{n-k-2}$ which implies that $\sigma^{k+1} \subset$ $\operatorname{Sing}\left(X^{n}\right)$. Repeating the argument above until $k+1=n-2$, one completes the proof.

\section{Gauss-Kronecker curvature and the deformation of convex domains.}

In this section, we discuss the deformation of convex domains and changes of Gauss-Kronecker curvature under the deformation. We also show that if $\Omega$ is a compact convex PL-domain $\Omega$ in a PL-manifold $X^{n}$ of non-positive curvature then the Gauss-Kronecker curvature measure of $\partial \Omega$ is supported by its vertices. 
We first discuss properties of convex subsets in $X^{n}$ as Federer did in [Fe1] for the Euclidean case.

Lemma 2.1. Let $\Omega_{0} \subset X^{n}$ be compact and convex. Then the set $\Omega_{s}=$ $\left\{x \in X^{n} \mid d\left(x, \Omega_{0}\right) \leq s\right\}$ is convex. Furthermore, for any $s \geq 0, t \geq 0$, $\Omega_{s+t}=\left(\Omega_{s}\right)_{t}$.

Proof. The first assertion follows from the fact that the function $f_{\Omega}(x)=$ $d(x, \Omega)$ is a convex function, as long as $X^{n}$ is a generalized Cartan-Hadamard space and $\Omega$ is convex.

The triangle inequality implies that $\left(\Omega_{s}\right)_{t} \subseteq \Omega_{s+t}$. To prove $\Omega_{s+t} \subseteq$ $\left(\Omega_{s}\right)_{t}$, it is sufficient to show that $d\left(y, \Omega_{s}\right)=t$ for every $y \in \partial \Omega_{s+t}$. This assertion is a direct consequence of the following fact. Let $\varphi_{\Omega, y}$ be a lengthminimizing geodesic segment of unit speed from $\Omega$ to $y$ for $y \notin \Omega$. When $\Omega$ is convex and $X^{n}$ is a generalized Cartan-Hardamard space, one can verify that $d\left(\Omega, \varphi_{\Omega, y}(t)\right)=t$, for $t \geq 0,(\operatorname{cf}[\mathrm{BH}])$. This completes the proof of Lemma 2.1 .

In the next Proposition we study the regularity properties of $\Omega_{s}$ for a convex subset $\Omega_{0} \subset X^{n}$.

Proposition 2.2. Let $\Omega_{0}$ be a compact, convex and nonempty subset of $X^{n}$, For $s>0,\left[\partial \Omega_{s}-\operatorname{Sing}\left(X^{n}\right)\right]$ is locally a $C^{1,1}$ sub-manifold of $\left[X^{n}-\operatorname{Sing}\left(X^{n}\right)\right]$ and its principle curvatures are locally bounded at twice differentiable points.

Proof. When $X^{n}=\mathbb{R}^{n}$, Proposition 2.2 was proved by Federer, (cf[Fe1]). It is sufficient to show that for each $y \in\left[\partial \Omega_{s}-\operatorname{Sing}\left(X^{n}\right)\right]$, the hypersurface $\partial \Omega_{s}$ is $C^{1,1}$ in a neighborhood of $y$. For this purpose, we use Lemma 2.1 and an earlier result of Federer.

Let $\pi_{s_{1}}: X^{n} \rightarrow \Omega_{s_{1}}$ be the nearest point projection. Choose $s_{1}<s$ sufficiently close to $s$. Suppose $\varphi_{y, \pi_{s_{1}}(y)}$ is the geodesic segment of unit speed from $y$ to $\pi_{s_{1}}(y)$ in $X^{n}$. By Lemma 2.1, we know that $\Omega_{s_{1}}$ is convex. Replacing $\Omega_{0}$ by $\Omega_{s_{1}}$ if needed, we may assume that $\varphi_{y, \pi_{s_{1}}(y)}$ does not intersect with $\operatorname{Sing}\left(X^{n}\right)$. Since the sets $\varphi_{y, \pi(y)}$ and $\operatorname{Sing}\left(X^{n}\right)$ are closed subsets of $X^{n}$ we let $\varepsilon_{0}=d\left(\varphi_{y, \pi(y)}, \operatorname{Sing}\left(X^{n}\right)\right)>0$ and $U_{\varepsilon}=\left\{x \in X^{n} \mid d\left(x, \varphi_{y, \pi(y)}\right)<\varepsilon\right\}$ for some $0<\varepsilon<\frac{\varepsilon_{0}}{2}$. Clearly, $U_{\varepsilon} \cap \operatorname{Sing}\left(X^{n}\right)=\varphi$. Smoothness is a local issue, thus we only have to verify that $\partial \Omega_{s} \cap U_{\varepsilon}$ is $C^{1,1}$. For this, we realize that $U_{\varepsilon}$ is isometric to a solid cylinder $B^{n-1}(\varepsilon) \times[0, s]$ attached with two half balls of radius $\varepsilon$. Therefore, we can isometrically embed $U_{\varepsilon}$ into the $n$-dimensional Euclidean space $\mathbb{R}^{n}$. Note that $d\left(U_{\varepsilon} \cap \partial \Omega_{s}, U_{\varepsilon} \cap \Omega_{0}\right)=s$. A result of Federer [Fe1, Theorem 4.8] and its proof imply that $U_{\varepsilon} \cap \partial \Omega_{s}$ is a locally $C^{1,1}$ submanifold. Furthermore, the principle curvatures of $\partial \Omega_{s} \cap U_{\varepsilon}$ are bounded by $\frac{4}{\varepsilon}$ at twice differentiable points. This finishes our proof. 
The classical Rademacher Theorem asserts that if $\left[\partial \Omega_{s}-\operatorname{Sing}\left(X^{n}\right)\right]$ is $C^{1,1}$ then it is twice differentiable almost everywhere. Proposition $1.11 \mathrm{im}$ plies that $\operatorname{Sing}\left(X^{n}\right) \cap \partial \Omega_{s}$ is a subset of zero $(n-1)$-dimensional measure in $\partial \Omega_{s}$. Thus the Gauss-Kronecker curvature $\widetilde{G K} \partial \Omega_{s}(p)$ of $\partial \Omega_{s}$ (with respect to the outward unit normal vector field) is well defined for almost all $p \in \partial \Omega_{s}$.

For any Borel set $V \subset X^{n}$, we consider the following function

$$
f_{\Omega, V}(s) \underset{\operatorname{def}}{=} \int_{V \cap\left[\partial \Omega_{s}-\operatorname{Sing}\left(X^{n}\right)\right]} \widetilde{G K} \partial \Omega_{s} d A,
$$

for $s>0$ where $\Omega_{0}$ is a convex subset of $X^{n}$.

The following proposition gives us a monotonicity property that will be useful in the definition of an outer measure for non-smooth convex domains.

Proposition 2.3. Let $\Omega_{0} \subset X^{n}$ be a compact, convex domain. Then for $0<s_{1}<s_{2}$ and Borel set $V \subset X^{n}$, we have

$$
\int_{V \cap\left[\partial \Omega_{s_{1}}-\operatorname{Sing}\left(X^{n}\right)\right]} \widetilde{G K}{\widetilde{G \Omega_{s_{1}}}} d A \leq \int_{\pi^{-1}(V) \cap\left[\partial \Omega_{s_{2}}-\operatorname{Sing}\left(X^{n}\right)\right]} \widetilde{G K}_{\partial \Omega_{s_{2}}} d A .
$$

where $\pi: X^{n} \rightarrow \Omega_{s_{1}}$ is the nearest point projection.

Proof. Since $X^{n}$ has non-positive curvature and $\Omega_{s_{1}}$ is convex, $\pi$ is a distance decreasing map. Since the set $\Sigma=\pi\left[\operatorname{Sing}\left(X^{n}\right) \cap\left(\overline{\Omega_{s_{2}}-\Omega_{s_{1}}}\right)\right] \subset$ $\pi\left(\operatorname{Sing}\left(X^{n}\right)\right) \cap \partial \Omega_{s_{1}}$ has zero $(n-1)$-dimensional measure in $\partial \Omega_{s_{1}}$, it follows that

$$
\int_{V \cap\left[\partial \Omega_{s_{1}}-\operatorname{Sing}\left(X^{n}\right)\right]} \widetilde{G K}_{\partial \Omega_{s_{1}}} d A=\int_{V \cap\left[\partial \Omega_{s_{1}}-\Sigma\right]} \widetilde{G K}_{\partial \Omega_{s_{1}}} d A .
$$

For any point $p \in\left[\partial \Omega_{s_{2}}-\pi^{-1}(\Sigma)\right]$, the geodesic segment $\varphi_{p, \pi(p)}$ from $p$ to $\pi(p)$ never hits the singular set $\operatorname{Sing}\left(X^{n}\right)$. Moreover, the set $U=\partial \Omega_{s_{1}}-\Sigma$ is a relative open in $\partial \Omega_{s_{1}}$, because $\Sigma$ is a closed subset. A direct computation shows that

$$
\begin{aligned}
& \int_{V \cap\left[\partial \Omega_{s_{1}}-\operatorname{Sing}\left(X^{n}\right)\right]} \widetilde{G K}_{\partial \Omega_{s_{1}}} d A=\int_{V \cap U} \widetilde{G K}_{\partial \Omega_{s_{1}}} d A \\
& =\int_{\pi^{-1}(V \cap U) \cap \partial \Omega_{s_{2}}} \widetilde{G K} \Omega_{\partial \Omega_{s_{2}}} d A \leq \int_{\pi^{-1}(V) \cap\left[\partial \Omega_{s_{2}}-\operatorname{Sing}\left(X^{n}\right)\right.} \widetilde{G K}_{\partial \Omega_{s_{2}}} d A .
\end{aligned}
$$

This completes the proof.

From Proposition 2.3 follows that $\int_{\pi^{-1}(V) \cap\left[\partial \Omega_{s}-\operatorname{Sing}\left(X^{n}\right)\right]} \widetilde{G K}_{\partial \Omega_{s}} d A$ is a monotone function of $s>0$. 
For any convex domain $\Omega$ (possibly with singularity) and a Borel set $V$, we define an outer measure by

$$
\int_{V \cap \partial \Omega} d\left(G K_{\partial \Omega}\right)=\lim _{s \rightarrow 0^{+}} \int_{\pi^{-1}(V) \cap\left[\partial \Omega_{s}-\operatorname{Sing}\left(X^{n}\right)\right]} \widetilde{G K}_{\partial \Omega_{s}} d A
$$

where $\pi: X \rightarrow \Omega$ is the nearest point projection.

In what follows, the notion of $\widetilde{G K}$ will be used for the classical Gauss-Kronecker curvature of $C^{1,1}$ hypersurfaces in the regular part of $\left[X^{n}-\operatorname{Sing}\left(X^{n}\right)\right]$. The notation of the measure $G K_{\partial \Omega}$ in $\int_{V \cap \partial \Omega} d\left(G K_{\partial \Omega}\right)$ is for subsets $V \cap \partial \Omega$, that possibly intersect the $\operatorname{singular} \operatorname{set} \operatorname{Sing}\left(X^{n}\right)$. In general the measure $G K_{\partial \Omega}$ is not absolutely continuous with respect to the $(n-1)$-dimensional Hausdorff measure. Therefore $\left.d G K_{\partial \Omega}\right|_{x} \neq f(x) d A$ for any bounded measurable function $f$ around corner points or singular points of $\partial \Omega$.

In the rest of this section, we discuss the upper semi-continuity of the outer measure $G K$ defined by equality (2.1). In the $n$-dimensional Euclidean space, Federer obtained a convergence result for curvature measures (see [Fe1]), which we now describe.

Suppose that $\Omega$ is a subset of $\mathbb{R}^{n}$. The reach of a subset $\Omega$ is the largest $\varepsilon$ (possibly $\infty$ ) such that if $x \in \mathbb{R}^{n}$ and the distance $d(x, \Omega)$ is smaller than $\varepsilon$, then $\Omega$ contains a unique point, $\pi_{\Omega}(x)$, nearest to $x$. Assuming that reach $(\Omega)>0$, Federer established the Steiner's type formula related to various curvature measures. For each bounded Borel subset $Q \subset \mathbb{R}^{n}$ and for $0 \leq \gamma<\operatorname{reach}(\Omega)$, the $n$-dimensional measure of $\left\{x \in \mathbb{R}^{n} \mid d(x, \Omega) \leq \gamma\right.$ and $\left.\pi_{\Omega}(x) \in Q\right\}$, is given by a polynomial of degree at most $n$ in $\gamma$, say, $\sum_{i=0}^{n} \gamma^{n-i} \alpha(n-i) \Phi_{i}(\Omega, Q)$, where $\alpha(j)=\operatorname{vol}_{j}\left(B^{j}(1)\right)$ is the $j$-dimensional measure of the unit $j$-sphere $S^{j}(1) \hookrightarrow \mathbb{R}^{j+1}$. The coefficients $\Phi_{j}(\Omega, Q)$ are countably additive with respect to $Q$, defining the curvature measures $\Phi_{0}(\Omega, \cdot), \Phi_{1}(\Omega, \cdot), \ldots, \Phi_{n}(\Omega, \cdot)$ (see [Fe1]). Federer's curvature measure $\Phi_{0}(\Omega, Q)$ is equal to our $\int_{\partial \Omega \cap Q} d\left(G K_{\partial \Omega}\right)$ up to a constant independent of $\Omega$ and $Q \subset \mathbb{R}^{n}$.

Recall that the Hausdorff metric between two sets $\Omega$ and $\Omega^{\prime}$ is defined as

$$
d_{H}\left(\Omega^{\prime}, \Omega\right)=\sup \left\{d\left(x^{\prime}, \Omega\right), d\left(\Omega^{\prime}, x\right) \mid x^{\prime} \in \Omega^{\prime}, x \in \Omega\right\} .
$$

Federer's Convergence Theorem [Fe1, p419] says the following.

Theorem 2.4. If a sequence of sets $\left\{\Omega_{j}\right\}$ in $\mathbb{R}^{n}$, all with reach at least $\varepsilon>0$, is convergent relative to the Hausdorff metric, then the associated 
sequences of curvature measures converge weakly to the curvature measure of the limit set $\Omega$, whose reach is also at least $\varepsilon$.

In particular, if $U \cap \partial \Omega_{j}$ and $U \cap \partial \Omega$ are locally $C^{1,1}$ hypersurfaces for some open set $U$ of $\mathbb{R}^{n}$, then

$$
\lim _{j \rightarrow \infty} \int_{V \cap \partial \Omega_{j}} \widetilde{G K}_{\partial \Omega_{j}} d A=\int_{V \cap \partial \Omega} \widetilde{G K} \partial \Omega d A,
$$

where $V$ is a Borel subset of $U \subset \mathbb{R}^{n}$.

If we replace $\mathbb{R}^{n}$ by a PL-manifold $X^{n}$ of non-positive curvature, the conclusion of Theorem 2.4 is not true for the outer measure $G K$ defined by equality (2.1). Therefore, we need to impose appropriate conditions on the sequence $\left\{\Omega_{j}\right\}$ in a PL-manifold $X^{n}$ in order to derive a weak convergence result. The following observation will be used to obtain our convergence result.

Lemma 2.5. Let $\Omega^{\prime} \subset \Omega$ be convex subsets in $X^{n}$. Suppose that $d_{H}\left(\Omega^{\prime}, \Omega\right) \leq$ $\varepsilon$. Then $d_{H}\left(\Omega_{s}^{\prime}, \Omega_{s}\right) \leq \varepsilon$ for any $s \geq 0$.

Proof. It is easy to see that $\Omega_{s}^{\prime} \subset \Omega_{s}$ because $\Omega^{\prime} \subset \Omega$. By the assumption that $d_{H}\left(\Omega, \Omega^{\prime}\right) \leq \varepsilon$, we see that $\Omega \subset \Omega_{\varepsilon}^{\prime}$. Using Lemma 2.1 we have $\Omega_{s} \subset$ $\left(\Omega_{\varepsilon}^{\prime}\right)_{s}=\Omega_{\varepsilon+s}^{\prime}=\left(\Omega_{s}^{\prime}\right)_{\varepsilon}$. It follows that $d_{H}\left(\Omega_{s}, \Omega_{s}^{\prime}\right) \leq \varepsilon$.

The following definition will be used in several sections of this paper.

Definition 2.6. (1) Let $\tau$ be a triangulation of $X^{n}, x \in X^{n}$. The open star of $x$ is the union of the interiors of cells containing $x$, denoted by $\operatorname{st}(x)$. The closed star of $x$ is the union of all $k$-simplexes $\bar{\sigma}^{k}$ such that $x \in \bar{\sigma}^{k}$. The closed star of $x$ is denote by $\operatorname{St}(x)$. Both $\operatorname{St}(x)$ and $\operatorname{st}(x)$ have the induced simplicial structure from $X^{n}$. If $\Omega \subset X^{n}$, we let

$$
\operatorname{St}(\Omega)=\bigcup_{x \in \Omega} \operatorname{St}(x), \quad \text { and } \quad \operatorname{st}(\Omega)=\bigcup_{x \in \Omega} \operatorname{st}(x) .
$$

(2) For $u \in T_{x}\left(X^{n}\right)$, we let $\sigma_{u}: t \rightarrow \operatorname{Exp}_{x}(t u)$ be the unique geodesic with $\sigma_{u}(0)=x$ and $\sigma_{u}^{\prime}(0)=u$ for $0 \leq t|u|<d(x, \partial[S t(x)])$. Let $A \subset X^{n}$ be a set and $a \in A$, the tangent cone of $A$ at $a$ is defined to be

$$
T_{a}(A)=\left\{u \mid u \in T_{a}\left(X^{n}\right), \liminf _{t \rightarrow 0^{+}} \frac{d\left(A, E x p_{a}(t u)\right)}{t}=0\right\} .
$$

(3) Let $\sigma^{k} \subset X^{n}$ be a $k$-dimensional simplex, with $k \geq 1$, and $q \in \sigma^{k}$ be a relative interior point of $\sigma^{k}$. Suppose that $\Omega$ is a convex domain with non-empty interior in $X^{n}, q \in \sigma^{k} \cap \partial \Omega$. Then we say $\partial \Omega$ is transversal to $\sigma^{k}$ at $q$ if there is non-zero vector $v \in T_{q}\left(\sigma^{k}\right)$ such that $\pm v \notin T_{q}(\partial \Omega)$. 
Lemma 2.7. Let $\Omega_{0}$ be a compact, convex subset in $X^{n}$. Let $\tau$ be a triangulation of $X^{n}$ and $N_{0}>0$. Then for all but finitely many $s \in\left[0, N_{0}\right]$, the boundary $\partial \Omega_{s}$ of the convex set $\Omega_{s}$ is transversal to $X^{(n-1)}$, the $(n-1)$ skeleton of $X^{n}$.

Proof. Let $X^{(0)}$ be the set of vertices of $X^{n}$, a discrete set. For almost all $s, \partial \Omega_{s} \cap X^{(0)}=\varphi$. For $k \geq 1$, we proceed as follows. Let $f(x)=d\left(x, \Omega_{0}\right)$ and $\pi: X^{n} \rightarrow \Omega_{0}$ be the nearest point projection. If $q \in \partial \Omega_{s} \cap \operatorname{Int}\left(\sigma^{k}\right)$ for some $s>0$ and $k$-simplex with $1 \leq k \leq n-1$, we consider the geodesic segment $\varphi_{q}:[0, s] \rightarrow X^{n}$ from $q$ to $\pi(q)$ and $v_{q}=\left(\varphi_{q}\right)_{\text {out }}^{\prime}(q)$ the tangent vector of $\varphi_{q}$ at $q$. If $\partial \Omega_{s}$ is not transversal to $\sigma^{k}$ at $q$, then the vector $v_{q}$ must be orthogonal to $\sigma^{k}$. It follows that $q$ is a critical point of the function $h_{\sigma^{k}}(y)=\left.f\right|_{\sigma^{k}}(y)$ for $y \in \operatorname{Int}\left(\sigma^{k}\right)$. Observe that $f(x)$ is a convex function. There is at most one critical value for $h_{\sigma^{k}}$, when $\sigma^{k}$ is given. Consequently, for a given $\sigma^{k}$ if $\partial \Omega_{s_{i}}$ is not transversal to $q_{i} \in \sigma^{k}, i=1,2$, then $s_{1}=s_{2}$ must hold. This is because both $s_{1}$ and $s_{2}$ are critical values of $h_{\sigma^{k}}(y)$ for $y \in \sigma^{k}$. Therefore, the cardinality of $s$ such that $\partial \Omega_{s}$ is not transversal to $X^{(n-1)}$ is less than or equal to the number of simplexes in $\operatorname{St}\left(\Omega_{s+N_{0}}\right)$. There are only finitely many simplexes intersecting with $\operatorname{St}\left(\Omega_{s+N_{0}}\right)$, because $\Omega_{s+N_{0}}$ is a compact set.

Let $s_{1}(\Omega)$ be the first non-zero critical value of the function $d(x, \Omega)$ when it is restricted to each simplex $\sigma^{k}$ in $S t(\Omega)$ for $k \geq 1$, i.e.,

$s_{1}(\Omega)=\sup \left\{s \mid \partial \Omega_{t}\right.$ is transversal to $\sigma^{k}, \sigma^{k} \cap \partial \Omega_{t} \neq \emptyset$, for all $\left.t \in(0, s)\right\}$.

For vertices of $X^{n}$, we let

$$
s_{0}(\Omega)=d\left(\bar{\Omega}, X^{(0)} \cap\left[X^{n}-\bar{\Omega}\right]\right),
$$

where $X^{(0)}$ is the set of vertices of $X^{n}$.

For convex domains $\Omega_{s}$ with $0<s<\min \left\{s_{0}(\Omega), s_{1}(\Omega)\right\}$, we shall study the support of the outer measure $G K_{\partial \Omega_{s}}$.

We emphasize that if $\partial \Omega$ has a corner point $p$, the Gauss-Kronecker measure $G K_{\partial \Omega}$ may be positive at $p \in \partial \Omega$, (see Theorem 2.12 below). Therefore, we first consider $\Omega_{s}$ with $\Omega_{s} \cap X^{(0)}=\emptyset$ instead.

Proposition 2.8. Let $\tau$ be a triangulation of $X^{3}, X^{(k)}$ the $k$-th skeleton of $X^{3}$ and let $\Omega$ be a compact convex domain. Let $s_{0}(\Omega)$ and $s_{1}(\Omega)$ be as above. Then for any $0<s<\hat{s}=\min \left\{s_{0}(\Omega), s_{1}(\Omega)\right\}$, the following is true:

(1) $\operatorname{Sing}\left(X^{3}\right) \cap \partial \Omega_{s}=\left\{q_{1}, \ldots, q_{m}\right\}$, where $q_{i} \in \sigma_{i}^{1} \subset \operatorname{Sing}\left(X^{3}\right)$.

(2) There exists $\hat{\epsilon}>0$ such that for all $0<\varepsilon<\hat{\epsilon}$, the equation

$$
\int_{\pi^{-1}\left(q_{i}\right) \cap\left[\partial \Omega_{s+\varepsilon}-\operatorname{Sing}\left(X^{3}\right)\right]} \widetilde{G K} \partial \Omega_{s+\varepsilon} d A=0
$$


holds. Hence, $\int_{q_{i}} d\left(G K_{\partial \Omega_{s}}\right)=0$ for each $i=1, \ldots, m$.

(3) Consequently, for any Borel set $V$ in $X^{3}$, the equation

$$
\int_{V \cap \partial \Omega_{s}} d\left(G K_{\partial \Omega_{s}}\right)=\int_{V \cap\left[\partial \Omega_{s}-\operatorname{Sing}\left(X^{3}\right)\right]} \widetilde{G K_{\partial \Omega_{s}}} d A
$$

holds, where $\widetilde{G K}_{\partial \Omega_{s}}$ is the classical Gauss-Kronecker curvature of the locally $C^{1,1}$ surface $\left[\partial \Omega_{s}-\operatorname{Sing}\left(X^{3}\right)\right]$.

Proof. (1) By our choice of s, the hypersurface $\partial \Omega_{s}$ is transversal to each 1simplex $\sigma_{j}^{1}$. Because $\partial \Omega_{s}$ is compact and $\operatorname{Sing}\left(X^{3}\right) \subset X^{(1)}$, the intersection $\operatorname{Sing}\left(X^{3}\right) \cap \partial \Omega_{s}$ is a compact discrete set. If $\operatorname{Sing}\left(X^{3}\right) \cap\left[\Omega_{\hat{s}}-\Omega\right]=\emptyset$, Proposition 2.8 holds trivially. We may assume that $\operatorname{Sing}\left(X^{3}\right) \cap\left[\Omega_{\hat{s}}-\Omega\right] \neq \emptyset$. By our definition of $\hat{s}$, the cardinality of the discrete set $\Lambda_{s}=\partial \Omega_{s} \cap \operatorname{Sing}\left(X^{3}\right)$ is independent of $s \in(0, \hat{s})$ and hence $\operatorname{Sing}\left(X^{3}\right) \cap \partial \Omega_{s}=\left\{q_{1}, \ldots, q_{m}\right\}$, where $q_{i} \in \sigma_{i}^{1} \subset \operatorname{Sing}\left(X^{3}\right)$.

(2) Let $\delta=\frac{1}{4} \min \left\{d\left(q_{i}, q_{j}\right) \mid q_{i} \neq q_{j}\right\}$. For each $\sigma_{i}^{1}$ above, we let $\ell_{i}(\varepsilon)$ be the length of $\sigma_{i}^{1} \cap\left[\Omega_{s+\varepsilon}-\Omega_{s}\right]$. Because of the transversal property, the function $\ell_{i}(\varepsilon)$ is a continuous function of $\varepsilon$. Therefore, there exists $0<\hat{\epsilon}<\delta$ such that $\max \left\{\ell_{i}(\hat{\epsilon}) \mid 1 \leq i \leq m\right\}<\delta$. In this case, for any pair $\sigma_{i}^{1} \neq \sigma_{j}^{1}$, the subset $\Sigma_{i}=\pi_{s}^{-1}\left(q_{i}\right) \cap\left[\Omega_{s+\hat{\epsilon}}-\Omega_{s}\right]$ does not meet $\sigma_{j}^{1}$. In other words, for any $p_{i} \in \pi_{s}^{-1}\left(q_{i}\right) \cap \partial \Omega_{s+\hat{\epsilon}}$, the geodesic segment from $p_{i}$ to $q_{i}$ does not meet $\operatorname{Sing}\left(X^{3}\right)$ except for the endpoint $q_{i}$.

For $0<\varepsilon<\hat{\epsilon}$, we consider the subset $\gamma_{i, \varepsilon}=\pi_{s}^{-1}\left(q_{i}\right) \cap \partial \Omega_{s+\varepsilon}$. As we pointed out above, $\gamma_{i, \varepsilon} \subset X^{3}-\operatorname{Sing}\left(X^{3}\right)$. Our goal is to show that $\gamma_{i, \varepsilon}$ is a smooth spherical arc of finite length for each i. To see this, for any $p \in \gamma_{i, \varepsilon}$ we let $\eta_{p}:[0, s+\varepsilon] \rightarrow X^{3}$ be a geodesic segment from $\pi_{0}\left(q_{i}\right)$ to $p$. By the definition of $\gamma_{i, \varepsilon}$, our geodesic segment $\eta_{p}$ passes through the singular point $q_{i}$ at time $t=s$. By Lemma 1.4, the geodesic $\eta_{p}$ satisfies the property $\angle\left(\left(\eta_{p}\right)_{\text {in }}^{\prime}\left(q_{i}\right),\left(\eta_{p}\right)_{\text {out }}^{\prime}\left(q_{i}\right)\right) \geq \pi$.

Let $\xi_{i}=\left(\eta_{p}\right)_{i n}^{\prime}\left(q_{i}\right)$. Note that $\xi_{i}$ is also equal to the initial vector of the geodesic from $q_{i}$ to $\pi_{0}\left(q_{i}\right) \in \Omega$. Thus, $\xi_{i}$ is independent of the choice of $p \in \gamma_{i, \varepsilon}$. Because each $\eta_{p}((s, s+\varepsilon]) \cap \operatorname{Sing}\left(X^{3}\right)=\emptyset$, the subset $\gamma_{i, \varepsilon}$ is isometric to the set

$$
\Gamma_{i}=\left\{w \in \operatorname{Link}\left(q_{i}, X^{3}\right) \mid d_{L}\left(w, \xi_{i}\right) \geq \pi\right\}
$$

up to a constant factor $\varepsilon$.

Recall that $q_{i} \in \sigma_{i}^{1} \subset \operatorname{Sing}\left(X^{3}\right)$. Thus, $\operatorname{Link}\left(q_{i}, X^{3}\right)=S^{0} *$ $\operatorname{Link}\left(\sigma_{i}^{1}, X^{3}\right)$. Let $v_{i}$ be the unit tangent vector of $\sigma_{i}^{1}$ at $q_{i}$ which points into $\Omega_{s}$, and let $\alpha_{i}=d_{L}\left(v_{i}, \xi_{i}\right)$. It follows from Lemma 1.1 that $\Gamma_{i}$ is 
a spherical arc of length equal to $\sin \left(\alpha_{i}\right)\left[\left|\operatorname{Link}\left(\sigma_{i}^{1}, X^{3}\right)\right|-2 \pi\right]$. Therefore, $\gamma_{i, \varepsilon}=\pi_{s}^{-1}\left(q_{i}\right) \cap \partial \Omega_{s+\varepsilon}$ is a smooth spherical arc of length equal to $\varepsilon \sin \left(\alpha_{i}\right)\left[\left|\operatorname{Link}\left(\sigma_{i}^{1}, X^{3}\right)\right|-2 \pi\right]$. This finishes the proof of (2).

The last assertion (3) is a direct consequence of (1)-(2).

In the next theorem we give conditions on a sequence of domains in order to assert that their total Gauss-Kronecker curvature converges.

Theorem 2.9. Let $\tau$ be a triangulation of $X^{3}, X^{(k)}$ the $k$-skeleton of $X^{3}$. Suppose that $\Omega \subset X^{3}$ is a compact, convex domain, $\hat{s}_{\Omega}$ is given by Proposition 2.8, $0<s<\hat{s}_{\Omega}$, and that $\{\Omega(i)\}_{i=1}^{+\infty}$ is a sequence of convex domains in $X^{3}$ satisfying

(1) $\lim _{i \rightarrow+\infty} \Omega(i)=\Omega_{s}$ in the Hausdorff metric;

(2) $\partial[\Omega(i)]-\operatorname{Sing}\left(X^{3}\right)$ is a $C^{1,1}$ hypersurface;

(3) $\int_{V \cap \partial[\Omega(i)]} d\left(G K_{\partial[\Omega(i)]}\right)=\int_{V \cap \partial[\Omega(i)]-\operatorname{Sing}\left(X^{3}\right)} \widetilde{G K}_{\partial[\Omega(i)]} d A$ for any Borel set $V$ in $X^{3}$, where $\widetilde{G K}$ stands for the classical Gauss-Kronecker curvature for a $C^{1,1}$ surface.

Then

$$
\lim _{i \rightarrow+\infty} \int_{V \cap \partial \Omega(i)} d\left(G K_{\partial[\Omega(i)]}\right)=\int_{V \cap \partial \Omega_{s}} d\left(G K_{\partial \Omega_{s}}\right) .
$$

for any Borel set $V$ in $X^{3}$.

Proof. Notice that if we choose $V \subset \operatorname{Sing}\left(X^{3}\right)$, the assumption (3) implies that $\int_{\operatorname{Sing}\left(X^{3}\right) \cap \partial[\Omega(i)]} d\left(G K_{\partial[\Omega(i)]}\right)=0$. Thus, we can choose the support of the outer measure $G K_{\partial[\Omega(i)]}$ within $X^{3}-\operatorname{Sing}\left(X^{3}\right)$, the regular part of $X^{3}$.

Since the outer measure $G K$ is additive, we first prove Theorem 2.9 for a special case when $d\left(\bar{V}, \operatorname{Sing}\left(X^{3}\right)\right) \geq 4 \delta>0$. Because $\partial \Omega_{s}$ is compact, for sufficiently large $\mathrm{i}$, we may assume that $d_{H}\left(\partial \Omega_{s}, \partial[\Omega(i)]\right) \leq \frac{\delta}{4}$. Let $\mathrm{W}$ be the $\delta$-neighborhood of $\partial \Omega_{s}$. For large i, the support of the measure $G K_{\partial[\Omega(i)]}$ lies within $W$, we may assume that $\bar{V}$ is compact.

Write $V$ as $V=\cup_{j=1}^{m} V_{j}$ where $V_{j}$ are Borel sets that satisfy

(a) $\left\{V_{j}\right\}_{j=1}^{m}$ are pairwise disjoint and

(b) the diameter of each $V_{j}$ is less than $\frac{\delta}{4}$, i.e., $\operatorname{diam}\left(V_{j}\right)<\frac{\delta}{4}$.

Thus, we can isometrically embedded each $U_{j}$ into $\mathbb{R}^{3}$ via a map $F_{j}$ : $U_{j} \rightarrow \mathbb{R}^{3}$, where $U_{j}$ is an open set of diameter less than $\frac{\delta}{4}$ and $V_{j} \subset U_{j}$. Let $\pi_{i}: X^{3} \rightarrow \Omega(i)$ be the nearest point project. In this case, for $0<$ $s<\frac{\delta}{4}$, we let $\left.W(i, j, s)=\left\{\pi_{i}^{-1}(\partial[\Omega(i)])\right\} \cap\left\{\partial[\Omega(i)]_{s}\right\} \cap V_{j}\right)$ and $\hat{W}(i, j, s)=$ 
$F_{j}(W(i, j, s))$. By Theorem 2.4 (Federer's Theorem) we have

$$
\begin{aligned}
& \lim _{i \rightarrow+\infty} \int_{V_{j} \cap \partial[\Omega(i)]} d\left(G K_{\partial[\Omega(i)]}\right)=\lim _{i \rightarrow+\infty} \int_{V_{j} \cap \partial[\Omega(i)]-\operatorname{Sing}\left(X^{3}\right)} \widetilde{G K} \partial[\Omega(i)] d A \\
& =\int_{V_{j} \cap \partial \Omega_{s}-\operatorname{Sing}\left(X^{3}\right)} \widetilde{G K_{\partial \Omega_{s}}} d A=\int_{V_{j} \cap \partial \Omega_{s}} d\left(G K_{\partial \Omega_{s}}\right) .
\end{aligned}
$$

This completes the proof of Theorem 2.9 for the case of $d\left(\bar{V}, \operatorname{Sing}\left(X^{3}\right)\right) \geq$ $4 \delta>0$ with some $\delta>0$.

For the general case of $V \subset\left[X^{3}-\operatorname{Sing}\left(X^{3}\right)\right]$ and any given $\epsilon>0$, we choose an open open set $U \supset \operatorname{Sing}\left(X^{3}\right)$ such that $\int_{U \cap \partial \Omega_{s}} d\left(G K_{\partial \Omega_{s}}\right)<\epsilon$. The later is possible because $\int_{\operatorname{Sing}\left(X^{3}\right) \cap \partial \Omega_{s}} d\left(G K_{\partial \Omega_{s}}\right)=0$.

Let $4 \delta=d\left(V-U, \operatorname{Sing}\left(X^{3}\right)\right)$. By the discussion above of the special case, we have

$$
\lim _{i \rightarrow+\infty} \int_{[V-U] \cap \partial[\Omega(i)]} \widetilde{G K}_{\partial[\Omega(i)]} d A=\int_{[V-U] \cap \partial \Omega_{s}} \widetilde{G K} \partial \Omega_{s} d A
$$

Therefore, we have

$$
\begin{aligned}
& \liminf _{i \rightarrow+\infty} \int_{V \cap \partial[\Omega(i)]} d\left(G K_{\partial[\Omega(i)]}\right) \\
& \geq \liminf _{i \rightarrow+\infty} \int_{[V-U] \cap \partial[\Omega(i)]} \widetilde{G K} \partial[\Omega(i)] \\
& =\lim _{i \rightarrow+\infty} \int_{[V-U] \cap \partial[\Omega(i)]} \widetilde{G K} \partial[\Omega(i)] \\
& =\int_{[V-U] \cap \partial \Omega_{s}} \widetilde{G K} \widetilde{G}_{\partial s} d A \\
& \geq \int_{V \cap \partial \Omega_{s}} d\left(G K_{\partial \Omega_{s}}\right)-\epsilon
\end{aligned}
$$

for any $\epsilon>0$. Thus, the inequality

$$
\liminf _{i \rightarrow+\infty} \int_{V \cap \partial[\Omega(i)]} d\left(G K_{\partial[\Omega(i)]}\right) \geq \int_{V \cap \partial \Omega_{s}} \widetilde{G K}_{\partial \Omega_{s}} d A
$$

holds.

Similarly we have

$$
\limsup _{i \rightarrow+\infty} \int_{[V-U] \cap \partial[\Omega(i)]} d\left(G K_{\partial[\Omega(i)]}\right) \leq \int_{V \cap \partial \Omega_{s}} \widetilde{G K} \widetilde{G \Omega}_{s} d A,
$$


for any open set $U \supset \operatorname{Sing}\left(X^{3}\right)$. Thus,

$$
\limsup _{i \rightarrow+\infty} \int_{\left[V-\operatorname{Sing}\left(X^{3}\right)\right] \cap \partial[\Omega(i)]} d\left(G K_{\partial[\Omega(i)]}\right) \leq \int_{V \cap \partial \Omega_{s}} \widetilde{G K}_{\partial \Omega_{s}} d A .
$$

Recall that $G K_{\partial[\Omega(i)]}$ and $G K_{\partial \Omega_{s}}$ are supported in $X^{3}-\operatorname{Sing}\left(X^{3}\right)$ by our assumption. This completes the proof of Theorem 2.9.

In what follows we show that the total Gauss-Kronecker curvature is lower semi-continuous.

Theorem 2.10. Suppose that there is a sequence of convex domains $\{\Omega(i)\}_{i=1}^{+\infty}$ in $X^{3}$ satisfying

(1) $\lim _{i \rightarrow+\infty} \Omega(i)=\Omega_{0}$ in the Hausdorff metric, where $\Omega_{0}$ is a compact and convex domain in $X^{n}$;

(2) For each $i, \Omega(i) \supset \Omega_{0}$ or $\Omega(i) \subset \Omega_{0}$ holds;

$$
\lim _{i \rightarrow+\infty} \int_{\partial[\Omega(i)]} d\left(G K_{\partial[\Omega(i)]}\right) \geq c
$$

Then

$$
\int_{\partial \Omega_{0}} d\left(G K_{\partial \Omega_{0}}\right) \geq c
$$

Proof. By Lemma 2.7, except for countably many $\left\{s_{j}\right\}_{j=1}^{\infty}$, we have that $X^{(0)} \cap \partial[\Omega(i)]_{s}=\emptyset$ and $\partial[\Omega(i)]_{s}$ is transversal to $\operatorname{Sing}\left(X^{3}\right)$ for all $\Omega(i)$. For such $s$, the proof of Proposition 2.8 implies that the equation

$$
\int_{V \cap \partial[\Omega(i)]_{s}} d\left(G K_{\partial[\Omega(i)]_{s}}\right)=\int_{V \cap \partial[\Omega(i)]_{s}-\operatorname{Sing}\left(X^{3}\right)} \widetilde{G K}_{\partial[\Omega(i)]_{s}} d A
$$

holds for any Borel set of $V$ in $X^{3}$.

Let $\hat{s}$ be given in Proposition 2.8, then for $0<s<\hat{s}_{\Omega}$, we also have

$$
\int_{V \cap \partial \Omega_{s}} d\left(G K_{\partial \Omega_{s}}\right)=\int_{V \cap \partial \Omega_{s}-\operatorname{Sing}\left(X^{3}\right)} \widetilde{G K}_{\partial \Omega_{s}} d A
$$

By Lemma 2.5, the sequence $\left\{[\Omega(i)]_{s}\right\}$ converges to $\Omega_{s}$ in Hausdorff topology. Therefore, for $s \notin\left\{s_{j}\right\}_{j=1}^{\infty}$ and $0<s<\hat{s}$, Theorem 2.9 yields

$$
\int_{\partial \Omega_{s}} d\left(G K_{\partial \Omega_{s}}\right)=\lim _{i \rightarrow+\infty} \int_{\partial[\Omega(i)]_{s}} d\left(G K_{\partial[\Omega(i)]_{s}}\right) .
$$


By our assumption (3) and Proposition 2.3, for each $\epsilon>0$, we have

$$
\int_{\partial[\Omega(i)]_{s}} d\left(G K_{\partial[\Omega(i)]_{s}}\right) \geq \int_{\partial[\Omega(i)]} d\left(G K_{\partial[\Omega(i)]}\right) \geq c-\epsilon
$$

for sufficiently large $i$. Hence, we conclude that

$$
\int_{\partial \Omega_{s}} d\left(G K_{\partial \Omega_{s}}\right)=\lim _{i \rightarrow+\infty} \int_{\partial[\Omega(i)]_{s}} d\left(G K_{\partial[\Omega(i)]_{s}}\right) \geq c-\epsilon
$$

where $s \notin\left\{s_{j}\right\}_{j=1}^{\infty}$ and $0<s<\hat{s}$. We now choose a sequence $\left\{s_{\alpha}\right\}$ such that $s_{\alpha} \rightarrow 0^{+}$but $s_{\alpha} \notin\left\{s_{j}\right\}_{j=1}^{\infty}$. Because of Proposition 2.3, letting $s=s_{\alpha} \rightarrow 0^{+}$ in the inequality above, we derive

$$
\int_{\partial \Omega} d\left(G K_{\partial \Omega}\right) \geq c-\epsilon
$$

Letting $\epsilon \rightarrow 0^{+}$we complete the proof.

To state our next theorem we need the following definition.

Definition 2.11. A domain $\Omega \subset X^{n}$ is called piecewise linear or briefly $\mathrm{PL}$ if there is a triangulation $\tau$ of $X^{n}$ such that $\left.\tau\right|_{\Omega}$ becomes a simplicial sub-complex.

In the next theorem we show that for any convex piecewise linear domain in a piecewise Euclidean manifold, the outer Gauss-Kronecker curvature measure is supported in the set of vertices of the domain.

Theorem 2.12. Let $\Omega \subset X^{n}$ be a compact convex $P L$ domain. Then

$$
\int_{\partial \Omega} d\left(G K_{\partial \Omega}\right)=\sum_{p \in(\partial \Omega)^{(0)}} \operatorname{vol}_{n-1}\left\{[\operatorname{Link}(p, \Omega)]^{*}\right\},
$$

where $Y^{(0)}$ denotes the 0 -th skeleton of the simplicial domain $Y$ and $A^{*}$ is the dual cone of $A, A^{*}=\left\{v \in \operatorname{Link}\left(p, X^{n}\right) \mid d_{L}(v, A) \geq \frac{\pi}{2}\right\}$.

Proof. We first show that

$$
\int_{[\partial \Omega]-(\partial \Omega)^{(0)}} d\left(G K_{\partial \Omega}\right)=0 .
$$

For each $q \in[\partial \Omega]-(\partial \Omega)^{(0)}$, we may assume that there is a $k$-dimensional simplex $\sigma^{k}$ of dimension $k \geq 1$ such that $q \in \operatorname{Int}\left(\sigma^{k}\right) \subset[\partial \Omega]$. When $q \in \operatorname{Int}\left(\sigma^{k}\right)$, there is a neighborhood of $q$ in the form of $W_{q}=U^{k} \times \mathcal{C}_{\varepsilon}\left(\operatorname{Link}\left(\sigma^{k}, X^{n}\right)\right)$, 
where $q \in U^{k} \subset \sigma^{k}, \mathcal{C}_{\varepsilon}\left(\operatorname{Link}\left(\sigma^{k}, X^{n}\right)\right)$ is the set of points in the normal cone at $q \in \sigma^{k}$ having distance to the vertex $q$ less than $\varepsilon$ and $\varepsilon=\varepsilon_{q}>0$ is a sufficiently small number depending on $q$, (cf. [CMS]). Let $\pi: X^{n} \rightarrow \Omega$ be the nearest point projection. For each $s$ with $0<s<\varepsilon_{q}$, one can see that $\left[\partial \Omega_{s}\right] \cap \pi^{-1}\left(U^{k}\right)$ is isometric to the product space $U^{k} \times V^{n-1-k}$ for some $(n-1-k)$ dimensional space $V^{n-1-k}$. It follows that

$$
\int_{\left[\partial \Omega_{s}\right] \cap \pi^{-1}\left(U^{k}\right)} d\left(G K_{\partial \Omega_{s}}\right)=0
$$

which implies the equality (2.4). Using it, one can easily verify that

$$
\int_{\partial \Omega} d\left(G K_{\partial \Omega}\right)=\int_{(\partial \Omega)^{(0)}} d\left(G K_{\partial \Omega}\right)=\sum_{p \in(\partial \Omega)^{(0)}} \operatorname{vol}_{n-1}\left\{[\operatorname{Link}(p, \Omega)]^{*}\right\} .
$$

\section{The geometry of $\partial \Omega_{s}$ for convex PL-domains $\Omega$.}

In this section we study the equidistance hypersurfaces $\partial \Omega_{s}$ for a compact convex PL-domain $\Omega$ in $X^{3}$. For $s>0$ small, we show that $\left[\partial \Omega_{s}-\operatorname{Sing}\left(X^{3}\right)\right]$ is a surface of piecewise constant curvature. We further show that the surface $\partial \Omega_{s}$ can be decomposed into at most four parts: spherical, cylindrical, conical and planar. When $X^{3}=\mathbb{R}^{3}$ and $\Omega \subset \mathbb{R}^{3}$ is a convex PL-domain the conical part never occurs in the decomposition of $\partial \Omega_{s}$. The conical part of $\partial \Omega_{s}$ might occur in the decomposition of $\partial \Omega_{s}$, if $\partial \Omega_{s}$ intersects with $\operatorname{Sing}\left(X^{3}\right)$ with an angle $\theta$ and $0<\theta<\frac{\pi}{2}$. The geometry of the hypersurface $\partial \Omega_{s}$ is closely related to the nearest point projection map $\pi_{\Omega}: X^{n} \rightarrow \Omega$. For any $x \in \partial \Omega_{s}$, we let $\varphi_{\pi_{\Omega}(x), x}$ be the geodesic segment from $\pi_{\Omega}(x)$ to $x$. Clearly, the initial direction $\varphi_{\pi_{\Omega}(x), x}^{\prime}(0)$ makes an angle with $\Omega$ at least $\frac{\pi}{2}$.

In this section we assume that all geodesic have unit speed.

Definition 3.1. We say that a geodesic $\varphi:[0, \ell) \rightarrow X^{n}$ is at least normal to $\Omega$, if $x_{0}=\varphi(0) \in \Omega$ and $d_{L}\left(\varphi_{\text {out }}^{\prime}\left(x_{0}\right), \operatorname{Link}\left(x_{0}, \Omega\right)\right) \geq \frac{\pi}{2}$, where $d_{L}$ denotes the distance function of $L=\operatorname{Link}\left(x_{0}, X^{3}\right)$.

Since $X^{3}$ has non-positive curvature and $\Omega$ is convex any geodesic ray $\varphi$, which is at least normal to $\Omega$, must satisfy $d(\varphi(s), \Omega)=s$ for $s \geq 0$. Hence, $\varphi$ intersects with $\partial \Omega_{s}$ at $\varphi(s)$. Therefore, we have

$$
\partial \Omega_{s}=\{\varphi(s) \mid \varphi \text { is a ray at least normal to } \Omega\} .
$$

This observation leads us to consider the moduli space of geodesic rays that are at least normal to $\Omega$. If $X^{3}$ has non-empty singular set and if a 
geodesic ray $\varphi$ might pass through $\operatorname{Sing}\left(X^{3}\right)$ at $\varphi\left(s_{0}\right)$, then $\varphi$ might bifurcate at $\varphi\left(s_{0}\right)$ in the following sense.

Definition 3.2. A geodesic segment $\varphi:[a, b] \rightarrow X^{n}$ of unit speed is said to bifurcate at $x_{0}=\varphi\left(s_{0}\right)$ with $a<s_{0}<b$, if there exist an $\epsilon>0$ and two geodesic segments $\psi_{i}:\left[s_{0}-\epsilon, s_{0}+\epsilon\right]$ such that $\left.\psi_{i}\right|_{\left[s_{0}-\epsilon, s_{0}\right]}=\left.\varphi\right|_{\left[s_{0}-\epsilon, s_{0}\right]}$ for $i=1,2 ;$ but $\left(\varphi_{1}\right)_{\text {out }}^{\prime}\left(x_{0}\right) \neq\left(\varphi_{2}\right)_{\text {out }}^{\prime}\left(x_{0}\right)$.

We let $\mathcal{G} e o_{\Omega,[0, s]}^{c a n}$ be the set of geodesic segments $\varphi:[0, s] \rightarrow X^{n}$ such that $\varphi$ is at least normal to $\Omega$ and $\varphi$ does not bifurcate at any $\varphi(t)$ with $t \in(0, s)$. Similarly, we let $\mathcal{G} e o_{\Omega,[0, s]}^{b i f}$ be the set of geodesic segments $\varphi:[0, s] \rightarrow X^{n}$ such that $\varphi$ is at least normal to $\Omega$ and $\varphi$ bifurcates at $\varphi(t)$ for some $t \in(0, s)$.

We decompose the annular set $\left[\Omega_{s}-\Omega\right]=\left[\Omega_{s}-\Omega\right]^{\text {can }} \cup\left[\Omega_{s}-\Omega\right]^{b i f}$, where

$$
\left[\Omega_{s}-\Omega\right]^{c a n}=\left\{\varphi(t) \mid 0<t \leq s, \varphi \in \mathcal{G} e o_{\Omega,[0, s]}^{c a n}\right\}
$$

and

$$
\left[\Omega_{s}-\Omega\right]^{b i f}=\left\{\varphi(t) \mid 0<t \leq s, \varphi \in \mathcal{G} e o_{\Omega,[0, s]}^{b i f}\right\} .
$$

Similarly, $\partial \Omega_{s}$ has a natural decomposition $\partial \Omega_{s}=\left(\partial \Omega_{s}\right)^{c a n} \cup\left(\partial \Omega_{s}\right)^{b i f}$, where

$$
\left(\partial \Omega_{s}\right)^{c a n}=\left[\Omega_{s}-\Omega\right]^{c a n} \cap \partial \Omega_{s}=\left\{\varphi(s) \mid \varphi \in \mathcal{G} e o_{\Omega,[0, s]}^{c a n}\right\}
$$

and

$$
\left(\partial \Omega_{s}\right)^{b i f}=\left[\Omega_{s}-\Omega\right]^{b i f} \cap \partial \Omega_{s}=\left\{\varphi(s) \mid \varphi \in \mathcal{G} e o_{\Omega,[0, s]}^{b i f}\right\} .
$$

If $0<s<d(p, \partial[S t(p)])$, we let $s \operatorname{Link}\left(p, X^{3}\right)=\left\{x \in X^{3} \mid d(x, p)=s\right\}$. If $A \subset \operatorname{Link}\left(p, X^{3}\right)$, we let $s A=\left\{s \varphi^{\prime}(0) \in s \operatorname{Link}\left(p, X^{3}\right) \mid \varphi(0)=p, \varphi_{\text {out }}^{\prime}(p) \in\right.$ $A, \varphi:[0, \ell] \rightarrow X^{3}$ is a geodesic $\}$. Similarly, if $A \subset\left[\operatorname{Link}\left(\sigma^{1}, \Omega\right)\right]$ and $p_{0} \in$ $\sigma^{1}$, we let $s A=\left\{s \varphi_{\text {out }}^{\prime}\left(p_{0}\right) \mid \varphi_{\text {out }}^{\prime}\left(p_{0}\right) \in A, \varphi(0)=p_{0}\right.$, where $\varphi:[0, \ell] \rightarrow$ $X^{3}$ is a geodesic $\}$. Clearly, the isometry type of the set $s A$ is independent of the choice of $p_{0} \in \sigma^{1}$. Furthermore, $s A$ is isometric to $A$ up to a constant scaling factor $\frac{1}{s}$.

Let $\partial \Omega=\bar{\Omega} \cap \overline{\left[X^{n}-\Omega\right]}$ and $\tau$ be a triangulation of $X^{3}$. When $\Omega$ is a convex, simplicial domain in $X^{3}$, we have $\partial \Omega_{s}=\cup_{\sigma^{k} \subset \partial \Omega}\left[\pi_{\Omega}^{-1}\left(\sigma^{k}\right) \cap \partial \Omega_{s}\right]$. Therefore, for each simplex $\sigma^{k} \subset \partial \Omega$, we study the sets $\pi_{\Omega}^{-1}\left(\sigma^{k}\right) \cap \partial \Omega_{s}$ in the next Proposition.

Proposition 3.3. Let $\Omega$ be a simplicial domain with respect to a triangulation $\tau, \delta_{\Omega}^{*}=d(\Omega, \partial[\operatorname{St}(\Omega)]), \mathcal{P}_{s}=\operatorname{Sing}\left(X^{3}\right) \cap\left[\Omega_{s}-\Omega\right]$ and $\pi=\pi_{\Omega}$ be as above. Suppose that $\tau^{\prime}$ is a refinement of $\tau$ such that $\pi_{\Omega}\left(\mathcal{P}_{s}\right) \subset(\partial \Omega)^{(1)}$, where $(\partial \Omega)^{(1)}$ is the 1-skeleton of $\partial \Omega$ with respect to $\tau^{\prime}$. Then for any $0<s<\delta_{\Omega}^{*}$ and any $k$-simplex $\sigma^{k} \subset \partial \Omega$ (with respect to $\tau^{\prime}$ ), the following assertions are true. 
(1) If $k=0$ and $q=\sigma^{0}$ is a vertex, then $\partial \Omega_{s} \cap \pi^{-1}(q)$ is isometric to a set in $s[\operatorname{Link}(q, \Omega)]^{*}$ where $[\operatorname{Link}(q, \Omega)]^{*}$ is the dual of $\operatorname{Link}(q, \Omega)$ in $\operatorname{Link}\left(q, X^{3}\right)$.

(2) If $k=1$, then $\pi^{-1}\left(\sigma^{1}\right) \cap\left(\partial \Omega_{s}\right)^{\text {can }}$ is isometric to a set in $s\left[\operatorname{Link}\left(\sigma^{1}, \Omega\right)\right]^{*} \times[0, \ell]$ of the cylinder $s\left[\operatorname{Link}\left(\sigma^{1}, X^{3}\right)\right] \times[0, \ell]$, where $\ell$ is the length of $\sigma^{1}$.

(3) If $k=2$, then $\pi^{-1}\left(\sigma^{2}\right) \cap\left[\Omega_{s}-\Omega\right]$ is isometric to $\sigma^{2} \times(0, s]$. Therefore, $\pi^{-1}\left(\sigma^{2}\right) \cap \partial \Omega_{s}$ is isometric to $\sigma^{2}$, and hence it is planar.

Proof. To show assertion (1) observe that if $x \in \partial \Omega_{s} \cap \pi^{-1}(q)$ and $\varphi_{q, x}$ be a geodesic segment from $q$ to $x$, then $\varphi_{q, x}$ must be at least normal to $\Omega$. Thus, $\varphi^{\prime}(0) \in[\operatorname{Link}(q, \Omega)]^{*}$. Conversely, if $\varphi_{q, x}$ is least normal to $\Omega$ at $q$, then by $\mathrm{CAT}(0)$ condition we obtain $d\left(\varphi_{q, x}(s), \Omega\right)=d\left(\varphi_{q, x}(s), q\right)$. Therefore, $x=\varphi_{q, x}(s) \in \partial \Omega_{s} \cap \pi^{-1}(q)$. This shows that $\partial \Omega_{s} \cap \pi^{-1}(q)$ is isometric to the set $s[\operatorname{Link}(q, \Omega)]^{*}$. Therefore, Assertion (1) is true.

In order to prove statement $(2)$ observe that $\pi^{-1}\left(\sigma^{1}\right) \cap\left(\partial \Omega_{s}\right)^{\text {can }}=$ $\left\{\varphi(s) \mid \varphi(0) \in \sigma^{1}, \varphi \in \mathcal{G}_{\left.e o_{\Omega,[0, s]}^{c a n}\right\}}\right\}$. Because each $\varphi \in \mathcal{G}_{e o_{\Omega,[0, s]}^{c a n}}$ does not intercept the singular set $\mathcal{P}_{s}$, the set $\pi^{-1}\left(\sigma^{1}\right) \cap \partial \Omega_{s}$ can be identified with the set $\Sigma=\left\{s \varphi^{\prime}(0) \mid \varphi(0) \in \sigma^{1}, \varphi \in \mathcal{G} e o_{\Omega,[0, s]}^{c a n}\right\}$. Since $\operatorname{Sing}\left(X^{3}\right)$ is a closed subset in $X^{3}$, for each $\varphi(s) \notin \overline{\mathcal{P}_{s}}$, there exists $\epsilon>0$ such that $B_{\epsilon}(\varphi(s)) \cap \overline{\mathcal{P}_{s}}=\emptyset$, where $B_{\epsilon}(\varphi(s))=\left\{p \in X^{3} \mid d(p, \varphi(s))<\epsilon\right\}$. It follows that the subset $\Sigma$ is a relatively open subset in $s\left[\operatorname{Link}\left(\sigma^{1}, \Omega\right)\right]^{*} \times[0, \ell]$, where $\ell=\left|\sigma^{1}\right|$ is the length of $\sigma^{1}$. Thus, the hypersurface $\pi^{-1}\left(\sigma^{1}\right) \cap\left(\partial \Omega_{s}\right)^{\text {can }}$ is isometric to a subset $\Sigma$ of the cylinder $s\left[\operatorname{Link}\left(\sigma^{1}, \Omega\right)\right] \times[0, \ell]$. The second assertion is verified.

To verify assertion (3) note that since $\pi\left(\mathcal{P}_{s}\right)=\pi_{\Omega}\left\{\operatorname{Sing}\left(X^{3}\right) \cap\left[\Omega_{s}-\Omega\right]\right\} \subset$ $(\partial \Omega)^{(1)}$ we have that $\pi^{-1}\left(\sigma^{2}\right) \cap \mathcal{P}_{s}=\emptyset$. Consequently, any geodesic segment $\varphi:[0, s] \rightarrow X^{3}$ normal to $\Omega$ with $\varphi(0) \in \sigma^{2}$ does not pass through the singular set $\mathcal{P}_{s}$. It follows that $\pi^{-1}\left(\sigma^{2}\right) \cap\left[\Omega_{s}-\Omega\right]$ is isometric to $\sigma^{2} \times(0, s]$. Moreover, the projection map: $\left.\pi_{\Omega}\right|_{\pi^{-1}\left(\sigma^{2}\right) \cap \partial \Omega_{s}}: \pi^{-1}\left(\sigma^{2}\right) \cap \partial \Omega_{s} \rightarrow \sigma^{2}$ is an one-to-one and onto map and $\left.\pi_{\Omega}\right|_{\pi^{-1}\left(\sigma^{2}\right) \cap \partial \Omega_{s}}$ is an isometry from $\pi^{-1}\left(\sigma^{2}\right) \cap$ $\partial \Omega_{s}$ to $\sigma^{2}$.

Let us now restate Proposition 3.3 in the following way.

Corollary 3.4. Let $\Omega$ be a simplicial domain with respect to a triangulation $\tau$ and $\delta_{\Omega}^{*}=d(\Omega, \partial[S t(\Omega)])$. Then, for $0<s<\delta_{\Omega}^{*}$, the canonical portion $\left(\partial \Omega_{s}\right)^{\text {can }}$ of $\partial \Omega_{s}$ consists of at most three parts: spherical, cylindrical and planar.

Proof. Let $\tau^{\prime}$ be a refinement of $\tau$ as in Proposition 3.3. Then we have 
$\left[\Omega_{s}-\Omega\right]^{\text {can }}=\cup_{k=0}^{2} \cup_{\sigma^{k}} \subset \partial \Omega\left\{\pi_{\Omega}^{-1}\left(\sigma^{k}\right) \cap\left[\Omega_{s}-\Omega\right]^{\text {can }}\right\}$. Corollary 3.4 now follows from Proposition 3.3.

In order to study the set $\left(\partial \Omega_{s}\right)^{b i f}$ we need the following definition.

Definition 3.5. (1) Let $\Omega \subset X^{n}$ be a simplicial domain with respect to a triangulation $\tau$. We define

$$
\hat{\delta}_{\sigma^{k}}=d\left(\bar{\sigma}^{k}, \partial\left[S t\left(\bar{\sigma}^{k}\right)\right]\right)=\min \left\{d\left(\sigma^{m}, \sigma^{k}\right) \mid \bar{\sigma}^{k} \cap \bar{\sigma}^{m}=\emptyset\right\}
$$

and $\hat{\delta}_{\Omega}=\min \left\{\delta_{\sigma^{k}} \mid \sigma^{k} \subset \overline{[S t(\Omega)-\Omega]}, 0 \leq k \leq n\right\}$, where $\bar{A}$ denotes the closure of the subset $A$ in $X^{n}$.

(2) Let $\mathcal{S}(\Omega)=[\operatorname{St}(\Omega)-\Omega] \cap \operatorname{Sing}\left(X^{3}\right) \neq \emptyset$ and $\Theta_{\Omega}=\min \left\{\theta^{*}\left(\sigma^{1}, \Omega\right) \mid \sigma^{1} \subset\right.$ $\left.\mathcal{S}(\Omega), \bar{\sigma}^{1} \cap \Omega \neq \emptyset\right\}$ where $\theta^{*}\left(\sigma^{1}, \Omega\right)=\min \left\{\frac{\pi}{2}, \theta\left(\sigma^{1}, \Omega\right)\right\}$ and $\theta\left(\sigma^{1}, \Omega\right)$ is the angle between $\bar{\sigma}^{1}$ and $\Omega$. We define

$$
\delta_{\Omega}=\frac{1}{3} \min \left\{1, \tan \Theta_{\Omega}\right\} \hat{\delta}_{\Omega}
$$

(3) If $\sigma_{i}^{1}$ is a singular line in $\mathcal{S}(\Omega)$ such that $\bar{\sigma}_{i}^{1}$ has an endpoint in $\partial \Omega$, we define $\sigma_{i, s}^{1}=\sigma_{i}^{1} \cap\left[\Omega_{s}-\Omega\right]$.

The following proposition is a basic observation about a subset of $\mathcal{S}(\Omega)$.

Proposition 3.6. Let $\Omega$ be a convex simplicial domain with respect to a triangulation $\tau, 0<s<\delta_{\Omega}$ and $\mathcal{P}_{s}=\left[\Omega_{s}-\Omega\right] \cap \operatorname{Sing}\left(X^{3}\right)=\cup_{i=1}^{m} \sigma_{i, s}^{1}$ be as in Definition 3.5. Suppose that $\partial \Omega \cap \bar{\sigma}_{i, s}^{1} \neq \partial \Omega \cap \bar{\sigma}_{j, s}^{1}$. Then $\pi_{\Omega}\left(\sigma_{i, s}^{1}\right) \cap \pi_{\Omega}\left(\sigma_{j, s}^{1}\right)=$ $\emptyset$.

Proof. Let $q_{i}=\bar{\sigma}_{i, s}^{1} \cap \partial \Omega$ for $i=1, \ldots m$. By our assumption, $q_{i}$ and $q_{j}$ are vertices of $X^{3}$ with respect to the given triangulation $\tau$. It follows from Definition 3.5 that $d\left(q_{i}, q_{j}\right) \geq \hat{\delta}_{\Omega}$ and $\theta^{*}\left(\sigma_{i, s}^{1}, \Omega\right) \leq \Theta_{\Omega}$. The length of the projection $\pi_{\Omega}\left(\sigma_{i, s}^{1}\right)$ is bounded by $\ell_{i, s}=s \cot \left[\theta^{*}\left(\sigma_{i, s}^{1}, \Omega\right)\right] \leq s \cot \left[\Theta_{\Omega}\right] \leq \frac{1}{3} \hat{\delta}_{\Omega}$ for each $i$. If it were true that two projections overlap, then we would have $d\left(q_{i}, q_{j}\right) \leq \ell_{i, s}+\ell_{j, s} \leq 2 \frac{1}{3} \hat{\delta}_{\Omega}$, which contradicts to our assumption that $d\left(q_{i}, q_{j}\right) \geq \hat{\delta}_{\Omega}$. Proposition 3.6 has been verified.

To further decompose $\left[\Omega_{s}-\Omega\right]^{b i f}$, for $\varphi \in \mathcal{G} e o_{\Omega,[0, s]}^{b i f}$, we let $t_{\varphi}=$ $\max \left\{t \mid \varphi(t) \in \mathcal{P}_{s}\right\}$, and $\mathcal{G} e o_{\sigma_{i, s}^{1}}^{\text {bif }}=\left\{\varphi \in \mathcal{G} e o_{\Omega,[0, s]}^{b i f} \mid \varphi\left(t_{\varphi}\right) \in \sigma_{i, s}^{1}\right\}$. The subset $\mathcal{G} e o_{\sigma_{i, s}^{1}}^{b i f}$ is non-empty if and only if $0<\theta\left(\bar{\sigma}_{i}^{1}, \Omega\right)<\frac{\pi}{2}$. Clearly, $\mathcal{G} e o_{\Omega,[0, s]}^{b i f}=\cup_{i=1}^{m} \mathcal{G} e o_{\sigma_{i, s}^{1}}^{b i f}$. We consider the following subset of $\left[\Omega_{s}-\Omega\right]^{b i f}$ :

$$
\mathcal{D}_{i, s}^{3}=\left\{\varphi(u) \mid t_{\varphi} \leq u \leq s, \varphi \in \mathcal{G} e o_{\sigma_{i, s}^{1}}^{b i f}\right\}
$$


and the corresponding hypersurface

$$
\mathcal{C}_{\sigma_{i, s}^{1}}=\mathcal{D}_{i, s}^{3} \cap \partial \Omega_{s}=\left\{\varphi(s) \mid \varphi \in \mathcal{G} e o_{\sigma_{i, s}^{1}}^{b i f}\right\} .
$$

By definition, we have $\left[\partial \Omega_{s}\right]^{b i f}=\cup_{i=1}^{m} \mathcal{C}_{\sigma_{i, s}^{1}}$.

In order to study the sets $\mathcal{C}_{\sigma_{i, s}^{1}}$ and $\mathcal{D}_{i, s}^{3}$ we need the following definition.

Definition 3.7. (1) A subset $\Sigma \subset X^{3}$ is said to be totally geodesic if $\Sigma$ is a convex subset of $X^{3}$.

(2) If $\triangle$ is a 2-dimensional totally geodesic subset in $X^{3}$ and if there is an isometric embedding $\Psi: \triangle \rightarrow \mathbb{R}^{2}$, then we define the boundary of $\triangle$ to be $\partial_{2} \triangle=\Psi^{-1}[\partial(\Psi(\triangle))]$.

(3) If $\triangle$ is a 2-dimensional totally geodesic subset in $X^{3}$ and if $\partial_{2} \triangle$ is a geodesic triangle in $X^{3}$, then we call $\triangle$ a 2-dimensional triangular surface (or briefly a 2-dimensional triangle) in $X^{3}$.

To describe the 2-dimensional triangles in $\mathcal{D}_{i, s}^{3}$ for each $i=1,2, \ldots, m$ we let $q_{i}=\bar{\sigma}_{i, s}^{1} \cap \partial \Omega, O_{i, s}=\bar{\sigma}_{i, s}^{1} \cap \partial \Omega_{s}$, and $\hat{O}_{i, s}=\pi_{\Omega}\left(O_{i, s}\right)$. We assume that $\theta_{i}=\theta\left(\bar{\sigma}_{i}^{1}, \Omega\right)$ satisfies $0<\theta_{i}<\frac{\pi}{2}$ for all $i=1,2, \ldots, m$.

In what follows we first show that the three points $\left\{q_{i}, O_{i, s}, \hat{O}_{i, s}\right\}$ span a 2-dimensional totally geodesic triangle $\hat{\triangle}_{i, s}$ in $X^{3}$. Then we show that there exists an family of 2-dimensional triangles $\left\{\triangle_{q_{i}, O_{i, s}, p_{u}}\right\}_{u \in \Gamma_{i}}$ in $\mathcal{D}_{i, s}^{3}$ such that each $\left\{\triangle_{q_{i}, O_{i, s}, p_{u}}\right\}$ intercepts $\hat{\triangle}_{i, s}$ at a common edge $\sigma_{i, s}^{1}$ with an angle at least $\pi$.

For this purpose, we derive an elementary criterion to assert when two triangles intercepts in a common edge with an angle at least $\pi$.

Lemma 3.8. Let $\sigma_{1}^{3}$ and $\sigma_{2}^{3}$ be 3-simplexes in $X^{3}$ with respect to a triangulation of $\tau$, and let $\triangle_{i} \subset \sigma_{i}^{3}$ be a 2-dimensional triangles for $i=1,2$. Suppose that $\triangle_{1} \cap \triangle_{2}=\sigma^{1} \subset \operatorname{Sing}\left(X^{3}\right)$. Then the angle between $\triangle_{1}$ and $\triangle_{2}$ at $\sigma^{1}$ is greater than or equal to $\pi$ if and only if there exists a geodesic $\varphi:(0, \ell) \rightarrow X^{3}$ such that $\varphi((0, \ell)) \subset \triangle_{1} \cup \triangle_{2}$ and $\varphi$ is transversal to $\sigma^{1}$ at $\varphi\left(s_{0}\right), 0<s_{0}<l$.

Proof. The Lemma follows by a straightforward application of Lemma 1.4 and Lemma 1.1. items (2) and (3).

An immediately application of Lemma 3.8 is the following corollary.

Corollary 3.9. Let $\left\{\triangle_{1}, \ldots, \triangle_{m^{\prime}}\right\}$ be a set of 2-dimensional triangles with a common vertex $q, \sigma_{j}^{3}$ be a 3-dimensional simplex and $\triangle_{j} \subset \sigma_{j}^{3}$ for $j=$ $1, \ldots m^{\prime}$. Suppose that $\triangle_{j}$ and $\triangle_{j+1}$ have a common edge $\sigma_{j}^{1}$ for $j=1, \ldots, m^{\prime}$ 
with each $\sigma_{j}^{1}$ different and $\sum_{j=1}^{m^{\prime}}\left|\operatorname{Link}\left(q, \triangle_{j}\right)\right|<\pi$. Then the following statements are equivalent:

(1) $\Psi=\cup_{1 \leq j \leq m^{\prime}} \operatorname{Link}\left(q, \triangle_{j}\right)$ is a spherical geodesic in $\operatorname{Link}\left(q, X^{3}\right)$;

(2) There is a geodesic segment $\varphi:[0, \ell] \rightarrow X^{3}$ such that $\varphi$ passes through each $\sigma_{j}$ at $\varphi\left(s_{j}\right)$ transversally for $j=1, \ldots m^{\prime}$;

(3) $\triangle_{j}$ and $\triangle_{j+1}$ meet at $\sigma_{j}^{1}$ with an angle $\geq \pi$ for $j=1, \ldots m^{\prime}$.

In the next Proposition we show that each $\mathcal{D}_{i, s}^{3}$ is a 3 -dimensional conical domain isometric to

$\tilde{\mathcal{D}}_{s, \theta_{0}, \eta}^{3}=\left\{(r \cos u \sin \theta, r \sin u \sin \theta, r \cos \theta) \mid 0 \leq \theta \leq \theta_{0}, 0 \leq r \leq r_{\theta}, 0 \leq u \leq \eta\right\}$,

where $r_{\theta}=\frac{s\left(\cot \theta_{0}\right)}{\cos \left(\theta_{0}-\theta\right)}$ and each $\mathcal{C}_{\sigma_{i, s}^{1}}$ is a conical hypersurface isometric to

$$
\tilde{\mathcal{C}}_{s, \theta_{0}, \eta}=\left\{\left(r \cos u \sin \theta_{0}, r \sin u \sin \theta_{0}, r \cos \theta_{0}\right) \mid 0 \leq r \leq s \cot \theta_{0}, 0 \leq u \leq \eta\right\} .
$$

Proposition 3.10. Let $\Omega$ be a simplicial convex domain in $X^{3}$ with respect to a triangulation. Suppose that $0<s<\delta_{\Omega}$ and $0<\theta_{i}<\frac{\pi}{2}$. Then there is an isometric immersion $\mathcal{F}_{i}: \mathcal{D}_{i, s}^{3} \rightarrow \tilde{\mathcal{D}}_{s, \theta_{i}, \eta_{i}}^{3}$ such that $\mathcal{F}_{i}\left(\mathcal{C}_{\sigma_{i, s}^{1}}\right) \subset \tilde{\mathcal{C}}_{s, \theta_{i}, \eta_{i}}$ where $\eta_{i}=\left|\operatorname{Link}\left(\sigma_{i}^{1}, X^{3}\right)\right|-2 \pi$ and $\theta_{i}$ is the angle between $\sigma_{i}^{1}$ and $\partial \Omega$.

Moreover, $\varsigma_{i}=\tilde{\mathcal{C}}_{s, \theta_{i}, \eta_{i}}-\mathcal{F}_{i}\left(\mathcal{C}_{\sigma_{i, s}^{1}}\right)$ is either empty or a union of finitely many straight line segments.

Proof. The singular line $\bar{\sigma}_{i, s}^{1}$ has two endpoints $q_{i} \in \partial \Omega$ and $O_{i} \in \partial \Omega_{s}$. Let $\hat{O}_{i}=\pi_{\Omega}\left(O_{i}\right)$. By Corollary 3.9, the three points $\left\{q_{i}, O_{i}, \hat{O}_{i}\right\}$ span a totally geodesic, 2-dimensional, rectangular triangle $\hat{\triangle}_{\tilde{\tau}}$ Because $\angle_{q_{i}}\left(O_{i}, \hat{O}_{i}\right)=\theta_{i}$, it follows that the length of $\sigma_{i, s}^{1}$ is equal to $\tilde{\ell}_{i}=\frac{s}{\sin \theta_{i}}$. Let $\tilde{\sigma}_{i, s}^{1}$ be the geodesic segment from $O_{i}$ to $q_{i}$. Observe that the two sets $\tilde{\sigma}_{i, s}^{1}$ and $\bar{\sigma}_{i, s}^{1}$ are equal as subsets, but they are viewed to have opposite orientations. Define $v_{i}=\left(\tilde{\sigma}_{i, s}^{1}\right)_{\text {out }}^{\prime}\left(O_{i}\right) \in L=\operatorname{Link}\left(O_{i}, X^{3}\right)$. Clearly, Link $\left(v_{i}, L\right)$ is isometric to $\operatorname{Link}\left(\sigma_{i}^{1}, X^{3}\right)$. If $\varphi_{O_{i}, \hat{O}_{i}}:[0, s] \rightarrow X^{3}$ is a geodesic segment from $O_{i}$ to $\hat{O}_{i}$, then we let $w_{i}=\left(\varphi_{O_{i}, \hat{O}_{i}}\right)_{\text {out }}^{\prime}\left(O_{i}\right)$. Because $\hat{\triangle}$ is a totally geodesic rectangular triangle, using Lemma 1.1 we obtain that $d_{L}\left(v_{i}, w_{i}\right)=\frac{\pi}{2}-\theta_{i}$.

Consider all spherical geodesics $\psi_{w_{i}, h}^{v_{i}}:\left[0, \frac{\pi}{2}\right] \rightarrow L$ from $w_{i}$ to $h$ with unit speed such that $\psi_{w_{i}, h}^{v_{i}}(0)=w_{i}, \psi_{w_{i}, h}^{v_{i}}\left(\frac{\pi}{2}-\theta_{i}\right)=v_{i}$ and $\psi_{w_{i}, h}^{v_{i}}\left(\frac{\pi}{2}\right)=h$. Let $\zeta_{\text {in }}=\left(\psi_{w_{i}, h}^{v_{i}}\right)_{i n}^{\prime}\left(v_{i}\right)$ and $\zeta_{h}=\left(\psi_{w_{i}, h}^{v_{i}, h}\right)_{\text {out }}^{\prime}\left(v_{i}\right)$. It follows from Lemma 1.4 that $\angle\left(\zeta_{\text {in }}, \zeta_{h}\right) \geq \pi$. Let $\Gamma_{i}=\left\{\zeta \in \operatorname{Link}\left(v_{i}, L\right) \mid \angle\left(\zeta_{\text {in }}, \zeta\right) \geq \pi\right\}$ and 
$\Gamma_{i}^{\prime}=\left\{\psi_{w_{i}, h}^{v_{i}}\left(\frac{\pi}{2}\right)\right\}$. By definition, $\Gamma_{i}^{\prime}$ is an arc in $\partial B_{\theta_{i}}\left(v_{i}\right)=\left\{h \in L \mid d\left(h, v_{i}\right)=\right.$ $\left.\theta_{i}\right\}$. There is an one-to-one and onto map $g: \Gamma_{i}^{\prime} \rightarrow \Gamma_{i}$ given by $g(h)=$ $\left(\psi_{w_{i}, h}^{v_{i}}\right)_{\text {out }}^{\prime}\left(v_{i}\right)$. Observe that $\left|\Gamma_{i}\right|=\eta_{i}=\left|\operatorname{Link}\left(\sigma_{i}^{1}, X^{3}\right)\right|-2 \pi$ and $\left|\Gamma_{i}^{\prime}\right|=$ $\left(\sin \theta_{i}\right) \eta_{i}$. Let $\zeta:\left[0, \eta_{i}\right] \rightarrow \Gamma_{i}$ be an arc-length parameterization of $\Gamma_{i}$. Consequently, there is a parameterization $h:\left[0, \eta_{i}\right] \rightarrow \Gamma_{i}^{\prime}$ given by $h(u)=$ $g^{-1}(\zeta(u))$.

Let $\xi_{u}:[0, \infty] \rightarrow X^{3}$ be a geodesic ray with $\xi_{u}(0)=O_{i}$ and $\left(\xi_{u}\right)_{\text {out }}^{\prime}\left(O_{i}\right)=h(u) \in \Gamma_{i}^{\prime} \subset \operatorname{Link}\left(O_{i}, X^{3}\right)$ for $u \in\left[0, \eta_{i}\right]$ and set $\max _{u}=$ $\sup \left\{t\left|\xi_{u}\right|_{(0, t)}\right.$ is not bifurcating $\}, t_{u}=\min \left\{s \cot \theta_{i}, \max _{u}\right\}$ and $p_{u}=\xi_{u}\left(t_{u}\right)$.

We assert the following is true.

Claim A (1) There exists $\varepsilon^{*}>0$ such that, for all $h(u) \in \Gamma_{i}^{\prime}, t_{u} \geq \varepsilon^{*}$.

(2) The three points $\left\{O_{i}, q_{i}, p_{u}\right\}$ span a totally geodesic 2-simplex $\triangle_{O_{i}, q_{i}, p_{u}}$ in $X^{3}$ with respect to a refinement of $\tau$;

(3) $\mathcal{D}_{i, s}^{3}=\cup_{u \in \Gamma_{i}} \triangle_{O_{i}, q_{i}, p_{u}}$.

(4) For all (except for possible finitely many) $u \in \Gamma_{i}$, the triangle $\triangle_{O_{i}, q_{i}, p_{u}}$ is a rectangular triangle with edge lengths $\left\{s, s \cot \theta_{i}, \frac{s}{\sin \theta_{i}}\right\}$.

Assuming that Claim A holds for a moment, we construct the isometric immersion $\mathcal{F}_{i}: \mathcal{D}_{i, s}^{3} \rightarrow \mathbb{R}^{3}$ as follows: We first define $\mathcal{F}\left(O_{i}\right)=(0,0,0)$, $\mathcal{F}_{i}\left(q_{i}\right)=\left(0,0, \tilde{\ell}_{i}\right) \mathcal{F}_{i}\left(\tilde{\sigma}_{i, s}^{1}(r)\right)=(0,0, r)$ for $r \in\left[0, \tilde{\ell}_{i}\right]$ where $\tilde{\ell}_{i}=\frac{s}{\sin \theta_{i}}$. Our next step is to define $\mathcal{F}_{i}: \Gamma_{i}^{\prime} \rightarrow S^{2}$, where $S^{2}$ is the unit sphere in $\mathbb{R}^{3}$. Define

$$
\mathcal{F}_{i}(h(u))=\left(\sin \theta_{i} \cos u, \sin \theta_{i} \sin u, \cos \theta_{i}\right)
$$

for $u \in\left[0, \eta_{i}\right]$, where $h:\left[0, \eta_{i}\right] \rightarrow \Gamma_{i}^{\prime}$ is a parameterization of $\Gamma_{i}^{\prime}$ of constant speed $\sin \theta_{i}$ as above. Define $\mathcal{F}_{i}\left(p_{u}\right)=t_{u} \mathcal{F}_{i}(h(u))$ and $\mathcal{F}_{i}\left(\xi_{u}(t)\right)=t \mathcal{F}_{i}(h(u))$ for all $t \in\left[0, t_{u}\right]$ and $u \in\left[0, \eta_{i}\right]$.

By Claim $\mathrm{A}(2)$, we know that $\triangle_{O_{i}, q_{i}, p_{u}}$ is a 2-simplex in $X^{3}$ with respect to some triangulation $\tau_{u}$ of $X^{3}$. We already defined $\mathcal{F}_{i}$ on the three vertices of $\triangle_{O_{i}, q_{i}, p_{u}}$. Therefore, we can linearly extend the map $\mathcal{F}_{i}$ to the whole triangle $\triangle_{O_{i}, q_{i}, p_{u}}$. Furthermore, the map $\mathcal{F}_{i}$ isometrically takes the triangle $\triangle_{O_{i}, q_{i}, p_{u}}$ into

$$
\triangle_{u}=\left\{\left(r \sin \theta \cos u, r \sin \theta \sin u, r \cos \theta_{i}\right) \mid 0 \leq r \leq r_{\theta}, 0 \leq \theta \leq \theta_{i}\right\},
$$

where $r_{\theta}=\frac{s\left(\cot \theta_{i}\right)}{\cos \left(\theta_{i}-\theta\right)}$ for $\theta \in\left[0, \theta_{i}\right]$. Theorem 3.10 now follows from Claim A.

It remains to verify Claim $\mathrm{A}$. Claim $\mathrm{A}(1)$ is a direct consequence of the fact that the exponential map $\operatorname{Exp}_{O_{i}}: B_{\varepsilon^{*}}^{*} \rightarrow X^{n}$ where $B_{r}^{*}=\{w \in$ $\left.T_{O}\left(X^{n}\right)|| w \mid \leq r\right\}$ is an isometric embedding provided that $\varepsilon^{*}$ is small (see $[\mathrm{BH}])$. 
To prove Claim A(2) consider the map $G_{i}:\left[X^{3}-\left\{O_{i}\right\}\right] \rightarrow \operatorname{Link}\left(O_{i}, X^{3}\right)$ given by $G_{i}(p)=\left(\varphi_{O_{i}, p}\right)_{\text {out }}^{\prime}\left(O_{i}\right)$, where $\varphi_{O_{i}, p}$ is a geodesic segment in $X^{3}$ from $O_{i}$ and $p$. Consider a subset $A=\left\{\psi_{w_{i}, h(u)}^{v_{i}}(\theta) \mid u \in\left[0, \eta_{i}\right], \frac{\pi}{2}-\theta_{i} \leq \theta \leq \frac{\pi}{2}\right\}$. If $\theta=\theta_{v}=d\left(v, v_{i}\right)$, we let $t_{v}=\min \left\{\max _{v}, \frac{s\left(\cot \theta_{i}\right)}{\cos \left(\theta-\theta_{i}\right)}\right\}$. Observe that, for any $\theta \in\left(\frac{\pi}{2}-\theta_{i}, \frac{\pi}{2}\right]$ and $v=\psi_{w_{i}, h(u)}^{v_{i}}(\theta)$, we have $\xi_{v}\left(\left(0, t_{v}\right)\right) \cap \operatorname{Sing}\left(X^{3}\right)=\emptyset$. Define $p_{v}=\xi\left(t_{v}\right)$. There are two cases for any given $u \in\left[0, \eta_{i}\right]$.

Case $2_{a}$. For every $v=\psi_{w_{i}, h(u)}^{v_{i}}(\theta)$ with $\theta \in\left(\frac{\pi}{2}-\theta_{i}, \frac{\pi}{2}\right], \max _{v} \geq \frac{s\left(\cot \theta_{i}\right)}{\cos \left(\theta-\theta_{i}\right)}$.

In this case, we have $\triangle_{O_{i}, q_{i}, p_{u}}=\left\{\xi_{v}(t) \mid 0 \leq t \leq t_{v}, v=\psi_{w_{i}, h(u)}^{v_{i}}(\theta)\right\}$. Therefore, the three points $\left\{O_{i}, q_{i}, p_{u}\right\}$ span a totally geodesic 2 -simplex $\triangle_{O_{i}, q_{i}, p_{u}}$ in $X^{3}$ with respect to a refinement of $\tau$ and hence Claim $\mathrm{A}(2)$ follows.

Case $2_{b}$. There exists some $v=\psi_{w_{i}, h(u)}^{v_{i}}(\theta)$ with $\theta \in\left(\frac{\pi}{2}-\theta_{i}, \frac{\pi}{2}\right]$ such that $\max _{v}<\frac{s\left(\cot \theta_{i}\right)}{\cos \left(\theta-\theta_{i}\right)}$ holds.

Using the spherical geodesic $\psi_{w_{i}, h(u)}^{v_{i}}$ and Proposition 3.8, one can show that, for sufficiently small $t$, the four points $\left\{\xi_{v}(t), q_{i}, O_{i}, \hat{O}_{i}\right\}$ span a totally geodesic 2-dimensional subset $\Sigma_{\xi_{v}(t)}$ in $X^{3}$. Because $\theta=\angle_{O_{i}}\left(\xi_{v}(t), \hat{O}_{i}\right) \leq \frac{\pi}{2}$, one can also show that $d_{\Sigma_{\xi_{v}(t)}}\left(\xi_{v}(t), \pi_{\Omega}\left(\sigma_{i, s}^{1}\right)\right) \leq s$. Because $\Sigma_{\xi_{v}(t)}$ is totally geodesic, we have that $d_{X^{3}}\left(\xi_{v}(t), \Omega\right) \leq s$ and $\pi_{\Omega}\left(\xi_{v}(t)\right) \in \pi_{\Omega}\left(\sigma_{i, s}^{1}\right)$, for all $t \leq$ $\frac{s\left(\cot \theta_{i}\right)}{\cos \left(\theta-\theta_{i}\right)}$. If $\xi_{v}\left(t_{v}\right) \in \operatorname{Sing}\left(X^{3}\right)$, then there exists $\sigma_{j, s}^{1}$ such that $\xi_{v}\left(t_{v}\right) \in \sigma_{j, s}^{1}$. By Proposition 3.6, $\bar{\sigma}_{j, s}^{1}$ and $\bar{\sigma}_{i, s}^{1}$ must have a common endpoint point $q_{i}$. It follows that $G_{i}\left(\sigma_{j, s}^{1}\right) \subset \psi_{w_{i}, h(u)}^{v_{i}}$. Thus, $\bar{\sigma}_{j, s}^{1}$ becomes an edge of the triangle $\triangle_{O_{i}, q_{i}, p_{u}}$. Furthermore, the set $\left\{O_{i}, q_{i}, p_{u}\right\}$ span a totally geodesic 2-simplex $\triangle_{O_{i}, q_{i}, p_{u}}$ in $X^{3}$ with respect to a refinement of $\tau$. Claim $\mathrm{A}(2)$ follows for Case $2_{b}$ as well.

To show Claim $\mathrm{A}(3)$, we use the proof of Claim $\mathrm{A}(2)$. The argument above shows that $\cup_{u \in \Gamma_{i}} \triangle_{O_{i}, q_{i}, p_{u}} \subset \mathcal{D}_{i, s}^{3}$. Conversely, if $p \in \mathcal{D}_{i, s}^{3}$, then we let $\hat{p}=\pi_{\Omega}(p) \in \hat{\sigma}_{i, s}^{1}$. It follows from Corollary 3.9 that the set $\left\{q_{i}, p, \hat{p}\right\}$ span a totally geodesic 2-dimensional set $\triangle_{q_{i}, p, \hat{p}}$. By definition of $\mathcal{D}_{i, s}^{3}$, the three points $\left\{q_{i}, p, O_{i}\right\}$ span a a totally geodesic 2-simplex $\triangle_{O_{i}, q_{i}, p}$ in $X^{3}$ with respect to a refinement of $\tau$. The subset $\Sigma_{p}=\triangle_{O_{i}, \hat{O}_{i}, q_{i}} \cup \triangle_{O_{i}, q_{i}, p}$ form a totally geodesic 2-dimensional subset in $X^{3}$. Let $\xi_{O_{i}, p}$ be the geodesic segment from $O_{i}$ to $p$, and let $v=\xi_{O_{i}, p}^{\prime}(0)$. Because $d\left(O_{i}, \hat{O}_{i}\right)=s \geq$ $d_{X^{3}}(p, \Omega)=d_{\Sigma_{p}}(p, \hat{p})$ and $X^{3}$ satisfies the $C A T(0)$ inequality, we obtain $\theta=\angle_{O_{i}}\left(p, \hat{O}_{i}\right)=d_{L}\left(v, w_{i}\right) \leq \frac{\pi}{2}$, where $w_{i}=\varphi_{O_{i}, \hat{O}_{i}}^{\prime}(0)$. Clearly, by definition of $\mathcal{D}_{i, s}^{3}$, one has $\theta \geq \frac{\pi}{2}-\theta_{i}$ and $t=d\left(p, O_{i}\right) \leq t_{v}$. Hence, $p=\xi_{v}(t)$ for some $v \in \psi_{w_{i}, h(u)}^{v_{i}}, h(u) \in \Gamma_{i}^{\prime}$ and $t \leq t_{v}$. It follows that $\mathcal{D}_{i, s}^{3} \subset \cup_{u \in \Gamma_{i}} \triangle_{O_{i}, q_{i}, p_{u}}$ and 
hence $\mathcal{D}_{i, s}^{3}=\cup_{u \in \Gamma_{i}} \triangle_{O_{i}, q_{i}, p_{u}}$. This completes the proof of Claim A (3).

For the last assertion of Claim A, observe that there are at most finitely many singular lines $\sigma_{j, s}^{1}$ in $\mathcal{P}_{s}$ with $\theta_{j} \in\left(0, \frac{\pi}{2}\right)$. Therefore, there are only finitely many geodesics $\xi_{u}$ that intercepts the singularities $\left\{\sigma_{j, s}^{1}\right\}$. Thus, for all (except for possible finitely many) $u \in \Gamma_{i}$, we have that $t_{u}=s \cot \theta_{i}$ and the triangle $\triangle_{O_{i}, q_{i}, p_{u}}$ is a rectangular triangle with edge lengths $\left\{s, s \cot \theta_{i}, \frac{s}{\sin \theta_{i}}\right\}$. Claim $\mathrm{A}(4)$ follows and so does Proposition 3.10.

We conclude this section by summarizing our main results above.

Theorem 3.11. Let $\Omega$ be a simplicial convex domain in $X^{3}$ with respect to a triangulation $\tau, \delta_{\Omega}$ be as in Definition 3.5 and $0<s<\delta_{\Omega}$. Then the hypersurface $\partial \Omega_{s}$ can be decomposed into two portions $\partial \Omega_{s}=\left(\partial \Omega_{s}\right)^{\text {can }} \cup$ $\left(\partial \Omega_{s}\right)^{\text {bif }}$ such that

(1) The portion $\left(\partial \Omega_{s}\right)^{\text {can }}$ consists at most three parts: spherical, cylindrical and planar.

(2) If there exists a singular 1-simplex $\sigma_{i}^{1} \subset[S t(\Omega)-\Omega]$ which intercepts $\Omega$ with angle $\theta_{i} \in\left(0, \frac{\pi}{2}\right)$, then $\left(\partial \Omega_{s}\right)^{\text {bif }}$ is a non-empty subset;

(3) The portion $\left(\partial \Omega_{s}\right)^{b i f}$ is a union of conic surfaces;

(4) If $\sigma_{i}^{1} \subset[\operatorname{St}(\Omega)-\Omega] \cap \operatorname{Sing}\left(X^{3}\right)$ and $O_{i}=\sigma_{i}^{1} \cap \partial \Omega_{s}$, then $\left|\operatorname{Link}\left(O_{i}, \partial \Omega_{s}\right)\right|=2 \pi+\left(\sin \theta_{i}^{*}\right)\left[\left|\operatorname{Link}\left(\sigma_{i}^{1}, X^{3}\right)\right|-2 \pi\right]$, where $\theta_{i}^{*}=$ $\min \left\{\theta_{i}, \frac{\pi}{2}\right\}$.

(5) If $p \in\left[\left(\partial \Omega_{s}\right)-\operatorname{Sing}\left(X^{3}\right)\right]$, then $\left|\operatorname{Link}\left(p, \partial \Omega_{s}\right)\right|=2 \pi$.

Proof. Assertions (1)-(3) are direct consequence of Corollary 3.4 and Proposition 3.10. For the remaining two assertions (3) and (4), we proceed as follows. For any $p \in \partial \Omega_{s}$, we let $\hat{p}=\pi_{\Omega}(p), \varphi_{p, \hat{p}}:[0, s] \rightarrow X^{3}$ be the unique geodesic segment from $p$ to $\hat{p}$ and $w_{p}=\left(\varphi_{p, \hat{p}}\right)_{\text {out }}^{\prime}(0)$. Because $\Omega$ is convex and $X^{3}$ satisfies the CAT(0) inequality, Corollary 3.4 and Proposition 3.10 imply that $\operatorname{Link}\left(p, \partial \Omega_{s}\right)=\left\{u \in L \mid d_{L}\left(u, w_{p}\right)=\frac{\pi}{2}\right\}$, where $L=\operatorname{Link}\left(p, X^{3}\right)$. When $p \notin \operatorname{Sing}\left(X^{3}\right)$, one knows that $\operatorname{Link}\left(p, X^{3}\right)$ isometric to the unit sphere $S^{2}$ in $\mathbb{R}^{3}$. In this case, the subset $\left\{u \in \operatorname{Link}\left(p, X^{3}\right) \mid d_{L}\left(u, w_{p}\right)=\frac{\pi}{2}\right\}$ is isometric to a great circle of length $2 \pi$ in $S^{2}$. It follows that $\left|\operatorname{Link}\left(p, \partial \Omega_{s}\right)\right|=2 \pi$ for $p \in\left[\left(\partial \Omega_{s}\right)-\operatorname{Sing}\left(X^{3}\right)\right]$. When $p=O_{i}=\sigma_{i}^{1} \cap \partial \Omega_{s}$ for some $\sigma_{i}^{1} \subset \operatorname{Sing}\left(X^{3}\right)$, we let $w_{i}=w_{p}$ and we keep the same notation as in the proof of Proposition 3.10. Let $\tilde{\sigma}_{i, s}^{1}:\left[0, \tilde{\ell}_{i}\right] \rightarrow X^{3}$ be the geodesic segment from $O_{i}$ to $q_{i}$, and let $v_{i}=\left(\tilde{\sigma}_{i, s}^{1}\right)_{\text {out }}^{\prime}\left(O_{i}\right)$. We already showed in the proof of Proposition 3.10 that $d_{L}\left(v_{i}, w_{i}\right)=\frac{\pi}{2}-\theta_{i}^{*}$, where $\theta_{i}^{*}=\min \left\{\theta_{i}, \frac{\pi}{2}\right\}$. Recall 
that $L=S^{0} * \operatorname{Link}\left(\sigma_{i}^{1}, X^{3}\right)$, where $S^{0}=\left\{v_{i},-v_{i}\right\}$. If $\theta_{i} \geq \frac{\pi}{2}$, then the proof of Proposition 3.3 (1) shows that $v_{i}=w_{i}$. Therefore, we have $\left\{u \in L \mid d_{L}\left(u, w_{i}\right)=\frac{\pi}{2}\right\}=\left\{u \in \operatorname{Link}\left(O_{i}, X^{3}\right) \mid d_{L}\left(u, v_{i}\right)=\frac{\pi}{2}\right\}$. Such a subset is isometric to $\operatorname{Link}\left(\sigma_{i}^{1}, X^{3}\right)$. Hence, $\left|\operatorname{Link}\left(O_{i}, \partial \Omega_{s}\right)\right|=\left|\operatorname{Link}\left(\sigma_{i}^{1}, X^{3}\right)\right|$ for the case $\theta_{i} \geq \frac{\pi}{2}$. When $0<\theta_{i}<\frac{\pi}{2}$, we let $-v_{i} \in \operatorname{Link}\left(O_{i}, \sigma_{i}^{1}\right)$ such that $-v_{i}$ is the opposite direction of $v_{i}$. It follows that $d\left(v_{i},-v_{i}\right)=\pi$ and $d\left(w_{i},-v_{i}\right) \geq \pi-\left[\frac{\pi}{2}-\theta_{i}^{*}\right]>\frac{\pi}{2}$. Let consider all spherical geodesics $\varphi_{w_{i}, u}:\left[0, \frac{\pi}{2}\right] \rightarrow L$ of length $\frac{\pi}{2}$ with the same initial point $w_{i}$. If $\varphi_{w_{i}, u}$ does not pass through $v_{i}$ then $\varphi_{w_{i}, u}$ does not bifurcate. Clearly, the subset $\Lambda_{i}=\left\{u \in \operatorname{Link}\left(O_{i}, X^{3}\right) \mid d_{L}\left(u, v_{i}\right)=\frac{\pi}{2}, \varphi_{w_{i}, u}\right.$ does not bifurcate $\}$ has length $2 \pi$. Let $\Gamma_{i}^{\prime}=\left\{u \in \operatorname{Link}\left(O_{i}, X^{3}\right) \mid d_{L}\left(u, v_{i}\right)=\frac{\pi}{2}, \varphi_{w_{i}, u}\right.$ passes through $\left.v_{i}\right\}$. The proof of Proposition 3.10 shows that the length $\left|\Gamma_{i}^{\prime}\right|$ of $\Gamma_{i}^{\prime}$ is equal to $\left(\sin \theta_{i}\right)\left[\left|\operatorname{Link}\left(\sigma_{i}^{1}, X^{3}\right)\right|-2 \pi\right]$. Therefore, we conclude that

$$
\left|\operatorname{Link}\left(O_{i}, \partial \Omega_{s}\right)\right|=\left|\Lambda_{i}\right|+\left|\Gamma_{i}^{\prime}\right|=2 \pi+\left(\sin \theta_{i}\right)\left[\left|\operatorname{Link}\left(\sigma_{i}^{1}, X^{3}\right)\right|-2 \pi\right]
$$

for the case $0<\theta_{i}<\frac{\pi}{2}$. The proof of Theorem 3.11 has been completed.

\section{A formula for the outer Gauss-Kronecker curvature in dimension 3, Proof of Main Theorem.}

Let $X^{n}$ be an $n$-dimensional PL manifold satisfying the CAT(0) condition and $\Omega \subset X^{n}$ be a bounded convex domain. In this section we calculate the total outer Gauss-Kronecker curvature of $\partial \Omega$ when $n=3$. Observe that in the 2-dimensional case the Gauss-Kronecker curvature is the geodesic curvature.

Theorem 4.1. Suppose that $\Omega \subset X^{2}$ is a compact, convex piecewise linear domain with boundary $\partial \Omega$. Then the total outer geodesic curvature of $\partial \Omega$ is given by

$$
\int_{\partial \Omega} d\left(G K_{\partial \Omega}\right)=2 \pi+\sum_{q_{i} \in \Omega \cap \operatorname{Sing}\left(X^{2}\right)}\left[\left|\operatorname{Link}\left(q_{i}, X^{2}\right)\right|-2 \pi\right] .
$$

Proof. It is proved in Theorem 2.12 that if $0<s<s_{0}=d(\Omega, \partial[S t(\Omega)])$ then

$$
\int_{\partial \Omega} d\left(G K_{\partial \Omega}\right)=\int_{\partial \Omega_{s}} \widetilde{G K} \widetilde{\partial}_{s} d \ell
$$

for $0<s<s_{0}$. Let $D_{\varepsilon}\left(q_{i}\right)$ be the metric disk of radius $\varepsilon$ centered at $q_{i}$, where $\left\{q_{1}, q_{2}, \ldots, q_{m}\right\}=\operatorname{Sing}\left(X^{2}\right) \cap \Omega, 0<\varepsilon<\frac{\delta}{4}$, and $\delta=\min \left\{s_{0}, d\left(q_{i}, q_{j}\right) \mid\right.$ 
$\left.q_{i} \in \operatorname{Sing}\left(X^{2}\right) \cap \bar{\Omega}, q_{i} \neq q_{j}\right\}$. The domain $\widehat{\Omega}=\Omega_{s}-\coprod_{i=1}^{m} D_{\varepsilon}\left(q_{i}\right)$ has no singularities. Using the Gauss-Bonnet formula we obtain

$$
2 \pi(1-m)=\int_{\partial \widehat{\Omega}} \widetilde{G K}_{\partial \widehat{\Omega}} d \ell=\int_{\partial \Omega_{s}} \widetilde{G K}_{\partial \Omega_{s}} d \ell-\sum_{i=1}^{m}\left|\operatorname{Link}\left(q_{i}, X^{2}\right)\right| .
$$

It follows that

$$
\int_{\partial \Omega} d\left(G K_{\partial \Omega}\right)=\int_{\partial \Omega_{s}} \widetilde{G K} \partial \Omega_{s} d \ell=2 \pi+\sum_{i=1}^{m}\left[\left|\operatorname{Link}\left(q_{i}, X^{2}\right)\right|-2 \pi\right] .
$$

In the following proposition we compute the total Gauss-Kronecker curvature of $\partial B_{s}^{3}(p)$.

Proposition 4.2. Let $\tau$ be a triangulation of $X^{3}$, and $p \in X^{3}$. Suppose that $\varepsilon_{0}=d(p, \partial[S t(p)])$. Then

$$
\begin{aligned}
\int_{\partial B_{s}(p)} d\left(G K_{\partial B_{s}(p)}\right) & =\operatorname{Area}\left(\operatorname{Link}\left(p, X^{3}\right)\right) \\
& =4 \pi+\sum_{\sigma^{1} \in S t(p)}\left[\left|\operatorname{Link}\left(\sigma^{1}, X^{3}\right)\right|-2 \pi\right]
\end{aligned}
$$

where $B_{s}=B_{s}(p)=\left\{x \in X^{3} \mid d(x, p) \leq s\right\}$ and $0<s<\varepsilon_{0}$.

Proof. Let $L=\operatorname{Link}\left(p, X^{3}\right) . \quad L$ is a piecewise spherical two-dimensional manifold. Thus the singularities of $L$ are isolated, say $v_{1}, \ldots, v_{m} \in \operatorname{Sing}(L)$. We choose a sufficiently small $\delta>0, \delta<\frac{1}{4} \min \left\{d_{L}\left(v_{i}, v_{j}\right) \mid v_{i} \neq v_{j}, v_{i}, v_{j} \in\right.$ Sing $(L)\}$. Let $D_{\delta}\left(v_{i}\right)=\left\{w \in L \mid d_{L}\left(w, v_{i}\right)<\delta\right\}$ be a metric disk centered at $v_{i}$ of radius $\delta$ in $L$. Then the surface $\Sigma_{\delta}=L-\bigcup_{i=1}^{m} D_{\delta}\left(v_{i}\right)$ is a smooth Riemannian surface of constant curvature $K \equiv 1$ and $\Sigma_{\delta}$ has its boundary $\partial \Sigma_{\delta}=\bigcup_{i=1}^{m} \partial D_{\delta}\left(v_{i}\right)$. Applying the Gauss-Bonnet theorem to $\Sigma_{\delta}$, we have

$$
\operatorname{Area}\left(\Sigma_{\delta}\right)=\int_{\Sigma_{\delta}} K_{\Sigma_{\delta}} d A=2 \pi(2-m)+(\cos \delta) \sum_{i=1}^{m}\left|\operatorname{Link}\left(v_{i}, L\right)\right|
$$

We now denote the $1-\operatorname{simplex} \varphi_{v_{i}}(t)=\operatorname{Exp}_{p}\left(t v_{i}\right)$ by $\sigma_{i}^{1}$ for $t \in\left[0, \varepsilon_{i}\right]$. The last formula can be rewritten as

$$
\text { Area }\left(\Sigma_{\delta}\right)=2 \pi(2-m)+\cos \delta \sum_{i=1}^{m}\left|\operatorname{Link}\left(\sigma_{i}^{1}, X^{3}\right)\right| .
$$

Letting $\delta \rightarrow 0$, we conclude

$$
\operatorname{Area}(L)=\lim _{\delta \rightarrow 0} \operatorname{Area}\left(\Sigma_{\delta}\right)=4 \pi+\sum_{i=1}^{m}\left[\left|\operatorname{Link}\left(\sigma_{i}^{1}, X^{3}\right)\right|-2 \pi\right]
$$


and hence our Proposition.

In order to state the next theorem we need the following definition.

Definition 4.3. Suppose that $\Omega$ is a compact domain with boundary and positive reach. Let $p \in \partial \Omega$ and $v \in\left\{\operatorname{Link}\left(p, X^{n}\right)-\operatorname{Link}(p, \Omega)\right\}$, the angle $\theta_{p}(v, \Omega)$ between $v$ and $\Omega$ is given by

$$
\theta_{p}(v, \Omega) \underset{\text { def }}{=} \varangle_{p}(v, \Omega) \underset{\text { def }}{=} d_{L}(v, \operatorname{Link}(p, \Omega))
$$

where $d_{L}$ stands for the distance function on $\operatorname{Link}\left(p, X^{n}\right)$. When $\operatorname{dim} X^{3}=$ $3, p \in \partial \Omega$ and $v \in\left\{\operatorname{Link}\left(p, X^{3}\right)-\operatorname{Link}(p, \Omega)\right\}$, we define

$$
\theta_{p}^{*}(v, \Omega)=\min \left\{\frac{\pi}{2}, \theta_{p}(v, \Omega)\right\} .
$$

The following theorem is a special case of our Main Theorem stated in the introduction.

Theorem 4.4. Let $\Omega \subset X^{3}$ be a convex $P L$ domain. Then

$$
\begin{aligned}
& \int_{\partial \Omega} d\left(G K_{\partial \Omega}\right)=4 \pi \\
& +\sum_{p \in(\partial \Omega)^{(0)}} \sum_{\sigma^{1} \subset S t(p)} \sum_{v \in \operatorname{Link}\left(p, \sigma^{1}\right)}\left[\left|\operatorname{Link}\left(\sigma^{1}, X^{3}\right)\right|-2 \pi\right] \sin \left[\theta_{p}^{*}(v, \Omega)\right] .
\end{aligned}
$$

Proof. Assume that $\partial \Omega_{s}$ meets $\sigma_{j}^{1} \subset \operatorname{Sing}\left(X^{3}\right)$ at $p_{j}$ for $j=1, \ldots, N^{\prime}$ and $0<s<\delta_{\Omega}$. After re-indexing if needed, we may assume that

(1) For $1 \leq j \leq m_{1}$, the singular 1 -simplex $\sigma_{j}^{1}$ meets $\partial \Omega$ at a vertex $v_{j}$ with angle less than $\frac{\pi}{2}$.

(2) For $m_{1}<j \leq m_{2}$, the singular 1 -simplex $\sigma_{j}^{1}$ meets $\partial \Omega$ at a vertex $v_{j}$ with angle exactly $\frac{\pi}{2}$.

(3) For $m_{2}<j \leq N^{\prime}$, the singular 1 -simplex $\sigma_{j}^{1}$ meets $\partial \Omega$ at a vertex $v_{j}$ with angle greater than $\frac{\pi}{2}$.

If $\sigma_{j}^{1}$ meets $\partial \Omega_{s}$ at a vertex $v_{j}$ with angle $\frac{\pi}{2}$, then by Corollary $3.7, p_{j}$ is contained in the intersection of the spherical region and the planar region of $\partial \Omega_{s}$. This intersection is along two spherical geodesics of length $r_{j}$ in $\partial \Omega_{s}$ with the same starting point $p_{j}$. Moreover the surface is $C^{1,1}$ around an intersection point.

Let us consider the metric disk $D_{\epsilon}\left(p_{j}\right)=\left\{p \in \partial \Omega_{s} \mid d_{\partial \Omega_{s}}\left(p, p_{j}\right)<\epsilon\right\}$. We choose $\epsilon<\frac{\pi}{2} s$ sufficiently small so that the disks $\left\{D_{\epsilon}\left(p_{j}\right)\right\}_{1 \leq j \leq N^{\prime}}$ are disjoint and the following extra conditions hold: 
(1) For $1 \leq j \leq m_{1}$, the disk $D_{\epsilon}\left(p_{j}\right)$ is contained entirely in the flat part of $\partial \Omega_{s}$;

(2) For $m_{1}<j \leq m_{2}, 0<\epsilon<r_{j}$;

(3) For $m_{2} \leq j \leq N^{\prime}$, the disk $D_{\epsilon}\left(p_{j}\right)$ is contained entirely in the spherical part of $\partial \Omega_{s}$;

(4) Each closed disk $\bar{D}_{\epsilon}\left(p_{j}\right)$ is Lipschitz homeomorphic to $\hat{D}_{\epsilon}\left(p_{j}\right)=\{w \in$ $\left.T_{p_{j}}\left(\partial \Omega_{s}\right)|| w \mid \leq \epsilon\right\}$.

In order to apply the Gauss-Bonnet formula to the surface

$$
M_{\epsilon}^{2}=\left[\partial \Omega_{s}-\cup_{1 \leq j \leq N^{\prime}}\left\{D_{\epsilon}\left(p_{j}\right)\right],\right.
$$

we need to estimate the total geodesic curvature of each $\partial D_{\epsilon}\left(p_{j}\right)$ in $\partial \Omega_{s}$. Let $\kappa_{\partial D_{\epsilon}\left(p_{j}\right)}(u)$ denote the geodesic curvature of $\partial D_{\epsilon}\left(p_{j}\right)$ with respect to the unit normal vector field pointing into $M_{\epsilon}^{2}$ (i.e., with respect to the outward unit normal vector of $D_{\epsilon}\left(p_{j}\right)$ along $\left.\partial D_{\epsilon}\left(p_{j}\right)\right)$.

When $1 \leq j \leq m_{1}$, the disk $D_{\epsilon}\left(p_{j}\right)$ is contained in the flat part of $\partial \Omega_{s}$. Hence, the total geodesic curvature of $\partial D_{\epsilon}\left(p_{j}\right)$ is given by

$$
\int_{\partial D_{\epsilon}\left(p_{j}\right)} \kappa_{\partial D_{\epsilon}\left(p_{j}\right)}(u) d \ell=\int_{u \in \operatorname{Link}\left(p_{j}, \partial \Omega_{s}\right)} \frac{1}{\epsilon} \epsilon d u=\left|\operatorname{Link}\left(p_{j}, \partial \Omega_{s}\right)\right| .
$$

When $m_{2} \leq j \leq N$, the disk $D_{\epsilon}\left(p_{j}\right)$ is contained in the spherical part of $\partial \Omega_{s}$. Hence, the total geodesic curvature of $\partial D_{\epsilon}\left(p_{j}\right)$ is given by

$$
\begin{aligned}
& \int_{\partial D_{\epsilon}\left(p_{j}\right)} \kappa_{\partial D_{\epsilon}\left(p_{j}\right)} d \ell \\
= & \int_{u \in \operatorname{Link}\left(p_{j}, \partial \Omega_{s}\right)}\left(\cot \frac{\epsilon}{s}\right) \sin \frac{\epsilon}{s} d u \\
= & \left(\cos \frac{\epsilon}{s}\right)\left|\operatorname{Link}\left(p_{j}, \partial \Omega_{s}\right)\right| .
\end{aligned}
$$

When $m_{1} \leq j \leq m_{2}$, by Corollary 3.7 and the discussion above, the disk $D_{\epsilon}\left(p_{j}\right)$ is divided into two parts by the two spherical geodesics in $\partial \Omega_{s}$ starting at the same point $p_{j}$. Let $\Sigma_{j, 0, \epsilon}$ be the flat part of $D_{\epsilon}\left(p_{j}\right)$ and let $\Sigma_{j, 1, \epsilon}$ be the spherical part of $D_{\epsilon}\left(p_{j}\right)$. Suppose that $\alpha_{j, 0}=\mid \operatorname{Link}\left(p_{j}, \Sigma_{j, 0, \epsilon} \mid\right.$ and $\alpha_{j, 1}=\mid \operatorname{Link}\left(p_{j}, \Sigma_{j, 1, \epsilon)} \mid\right.$. Clearly, we have

$$
\alpha_{j, 0}+\alpha_{j, 1}=\left|\operatorname{Link}\left(p_{j}, \partial \Omega_{s}\right)\right| .
$$


A computation shows that

$$
\int_{\Sigma_{j, 0, \epsilon} \cap \partial D_{\epsilon}\left(p_{j}\right)} \kappa_{\partial D_{\epsilon}\left(p_{j}\right)}(u) d \ell=\alpha_{j, 0}
$$

and

$$
\int_{\Sigma_{j, 1, \epsilon} \cap \partial D_{\epsilon}\left(p_{j}\right)} \kappa_{\partial D_{\epsilon}\left(p_{j}\right)}(u) d \ell=\alpha_{j, 1} \cos \frac{\epsilon}{s} .
$$

It follows from (4.3)-(4.7) that

$$
\left|\operatorname{Link}\left(p_{j}, \partial \Omega_{s}\right)\right| \cos \frac{\epsilon}{s} \leq \int_{\partial D_{\epsilon}\left(p_{j}\right)} \kappa_{\partial D_{\epsilon}\left(p_{j}\right)}(u) d \ell \leq\left|\operatorname{Link}\left(p_{j}, \partial \Omega_{s}\right)\right|
$$

holds for all $1 \leq j \leq N^{\prime}$.

Corollary 3.7 implies that the surface $M_{\epsilon}^{2} \subset\left[\partial \Omega_{s}-\operatorname{Sing}\left(X^{3}\right)\right]$ is $C^{1,1}$. Thus we can apply the Gauss-Bonnet Theorem to $M_{\epsilon}^{2} \subset \partial \Omega_{s}$. The Euler number of $M_{\epsilon}^{2}$ is $2-N^{\prime}$. By Corollary 3.7, the intrinsic Gauss curvature of $M_{\epsilon}^{2}$ is either $\frac{1}{s^{2}}$ or 0 . Therefore, we conclude that

$$
\int_{M_{\epsilon}^{2}} \widetilde{G K}_{\partial \Omega_{s}}=2 \pi\left(2-N^{\prime}\right)+\sum_{1 \leq j \leq N^{\prime}} \int_{\partial D_{\epsilon}\left(p_{j}\right)} \kappa_{\partial D_{\epsilon}\left(p_{j}\right)}(u) d \ell .
$$

It follows from inequalities (4.8) and (4.9) that

$$
\begin{aligned}
& 2 \pi\left(2-N^{\prime}\right)+\sum_{1 \leq j \leq N^{\prime}}\left|\operatorname{Link}\left(p_{j}, \partial \Omega_{s}\right)\right| \cos \frac{\epsilon}{s} \\
\leq & \int_{M_{\epsilon}^{2}} \widetilde{G K} \partial \Omega_{s} d A \\
\leq & 2 \pi\left(2-N^{\prime}\right)+\sum_{1 \leq j \leq N^{\prime}}\left|\operatorname{Link}\left(p_{j}, \partial \Omega_{s}\right)\right| .
\end{aligned}
$$

Letting $\epsilon \rightarrow 0$ in (4.10) and using Corollary 3.7, we arrive at

$$
\begin{aligned}
& \int_{\partial \Omega_{s}-\operatorname{Sing}\left(X^{3}\right)} \widetilde{G K}_{\partial \Omega_{s}} d A \\
= & 2 \pi\left(2-N^{\prime}\right)+\sum_{1 \leq j \leq N^{\prime}}\left|\operatorname{Link}\left(p_{j}, \partial \Omega_{s}\right)\right| \\
= & 4 \pi+\sum_{1 \leq j \leq N^{\prime}} \eta_{j} \sin \theta_{p_{j}}^{*}\left(\sigma_{j}^{1}, \partial \Omega_{s}\right),
\end{aligned}
$$


where $\eta_{j}=\left|\operatorname{Link}\left(\sigma_{i}^{1}, X^{3}\right)\right|-2 \pi$. Letting $s \rightarrow 0$ in (4.11), one completes the proof of Theorem 4.4.

The proof of the Main Theorem can be reduced to our previous theorem. Proof of Main Theorem. For each $0<s<\hat{s}$ be as in Proposition 2.8. Assume that there exists a sequence of compact convex PL-domains $\{W(i, s)\}$ with non-empty interior such that $W(i, s) \subset \Omega_{s}$ and $\lim _{i \rightarrow \infty} W(i, s)=\Omega_{s}$ in the Hausdorff metric. The existence of these sets will be discuss later. Theorem 4.4 and its proof imply that

$$
\begin{aligned}
& \int_{\partial W(i, s)} d\left(G K_{\partial W(i, s)}\right)=4 \pi+ \\
& \sum_{p \in \partial W(i, s)} \sum_{\sigma^{1} \subset S t(p)} \sum_{v \in \operatorname{Link}\left(p, \sigma^{1}\right)}\left[\left|\operatorname{Link}\left(\sigma^{1}, X^{3}\right)\right|-2 \pi\right] \sin \left[\theta_{p}^{*}(v, W(i, s))\right] .
\end{aligned}
$$

For sufficiently large $i$, we may assume that $\Omega_{s / 2} \subset W(i, s) \subset \Omega_{s}$.

For each given $(i, s)$, by the proofs of Theorem 3.11 and Theorem 4.4, there exists a $\delta_{i, s}$ such that as long as $0<\epsilon_{i}<\delta_{i, s}$, we have

$$
\begin{aligned}
& \int_{\partial[W(i, s)]_{\epsilon_{i}}} d\left(G K_{\partial[W(i, s)]_{\epsilon_{i}}}\right)=4 \pi \\
& +\sum_{p \in \partial[W(i, s)]_{\epsilon_{i}}} \sum_{\sigma^{1} \subset S t(p)} \sum_{v \in \operatorname{Link}\left(p, \sigma^{1}\right)}\left[\left|\operatorname{Link}\left(\sigma^{1}, X^{3}\right)\right|-2 \pi\right] \sin \left[\theta_{p}^{*}\left(v,[W(i, s)]_{\epsilon_{i}}\right)\right] .
\end{aligned}
$$

Let us fix a sufficiently small $s$. By Lemma 2.7, except for countably many $\left\{\delta_{\alpha}\right\}$, we have $\partial[W(i, s)]_{\epsilon_{i}} \cap X^{(0)}=\emptyset$ and $\partial[W(i, s)]_{\epsilon_{i}}$ is transversal to $\operatorname{Sing}\left(X^{3}\right)$ for all for $i=1,2, \ldots \ldots$

Choose a sequence $\left\{\epsilon_{i}\right\}$ such that $\epsilon_{i} \rightarrow 0+$ and $\epsilon_{i} \notin\left\{\delta_{\alpha}\right\}$. By Lemma 2.5 , we have

$$
0 \leq \lim _{i \rightarrow \infty} d_{H}\left([W(i, s)]_{\epsilon_{i}}, \Omega_{s+\epsilon_{i}}\right) \leq \lim _{i \rightarrow \infty} d_{H}\left(W(i, s), \Omega_{s}\right)=0 .
$$

Thus, $\lim _{i \rightarrow \infty}[W(i, s)]_{\epsilon_{i}}=\Omega_{s}$. Using Theorem 2.9 and letting $i \rightarrow \infty$ in (4.13), we get

$$
\begin{aligned}
& \int_{\partial \Omega_{s}} d\left(G K_{\partial \Omega_{s}}\right)=4 \pi \\
& +\sum_{p \in\left(\partial \Omega_{s}\right)} \sum_{\sigma^{1} \subset S t(p)} \sum_{v \in \operatorname{Link}\left(p, \sigma^{1}\right)}\left[\left|\operatorname{Link}\left(\sigma^{1}, X^{3}\right)\right|-2 \pi\right] \sin \left[\theta_{p}^{*}\left(v, \Omega_{s}\right)\right]
\end{aligned}
$$


Letting $s \rightarrow 0$ in (4.14), we complete the proof of Main Theorem under the assumption that the desired family $\{W(i, s)\}$ exist.

It remains to construct the subsets $\{W(i, s)\}$. Let $\tau_{0}$ be a triangulation of $X^{3}$. Let $\hat{s}$ as in Proposition 2.8, so that $\partial \Omega_{s}$ is transversal to each $\sigma^{k}$ for $k=0,1,2$. The convexity of $\partial \Omega_{s}$ implies that $\sigma_{j}^{1} \cap \partial \Omega_{s}$ is a discrete subset of at most two points. If $\sigma_{j}^{1} \cap \partial \Omega_{s} \neq \emptyset$. we let $\sigma_{j}^{1} \cap \partial \Omega_{s}=\left\{p_{1, j, h}\right\}_{h=1}^{m_{1, j}}$, where $1 \leq m_{1, j} \leq 2$. Re-triangulate $\bar{\sigma}_{j}^{1}$ so that $\left\{p_{1, j, h}\right\}_{h=1}^{m_{1, j}}$ become vertices and the mesh size of this new division of $\bar{\sigma}_{j}^{1}$ is less than $\frac{1}{i}$. The convexity of $\partial \Omega_{s}$ implies that $\sigma_{j}^{2} \cap \partial \Omega_{s}$ is a union of at most three connected arcs. Thus, we let $\sigma_{j}^{2} \cap \partial \Omega_{s}=\left\{\gamma_{2, j, h}\right\}_{h=1}^{m_{2, j}}$, where $1 \leq m_{1, j} \leq 3$ when $\sigma_{j}^{2} \cap \partial \Omega_{s} \neq \emptyset$. Notice that the endpoints of each connected arc $\gamma_{2, j, h}$ are contained in $\cup_{j}\left\{p_{1, j, h}\right\}_{h=1}^{m_{1, j}}$. Divide each connected arc $\bar{\gamma}_{2, j, h}$ into finitely pieces by adding new points $\left\{p_{2, j, h}\right\}_{h=1}^{m_{2, j}}$ such that the distance between consecutive points is less than $\frac{1}{i}$. Re-triangulate $\bar{\sigma}_{j}^{2}$ so that $\left\{p_{2, j, h}\right\}_{h=1}^{m_{2, j}} \cup\left\{\left(\bar{\sigma}_{j}^{2}\right) \cap\left[\cup_{n}\left\{p_{1, n, h}\right\}_{h=1}^{m_{1, n}}\right]\right\}$ become vertices and the mesh size of this new triangulation of $\bar{\sigma}_{j}^{2}$ is less than $\frac{1}{i}$. The convexity of $\partial \Omega_{s}$ implies that $\sigma_{j}^{3} \cap \partial \Omega_{s}$ is a union of at most four connected topological disks. If $\sigma_{j}^{3} \cap \partial \Omega_{s} \neq \emptyset$ we let $\sigma_{j}^{3} \cap \partial \Omega_{s}=\left\{D_{3, j, h}\right\}_{h=1}^{m_{3, j}}$, where $1 \leq m_{1, j} \leq 4$. Notice that the boundary of each topological disk $D_{3, j, h}$ are contained in $\cup_{n}\left\{\gamma_{2, n, h}\right\}_{h=1}^{m_{2, n}}$, which were described above. For topological disk $D_{3, j, h}$, we add more points $\left\{p_{3, j, h}\right\}_{h=1}^{m_{3, j}}$ such that $\left\{p_{3, j, h}\right\}_{h=1}^{m_{3, j}} \cup\left\{\left(\bar{\sigma}_{j}^{3}\right) \cap\right.$ $\left.\left[\cup_{k=1}^{2} \cup_{n}\left\{p_{k, n, h}\right\}_{h=1}^{m_{k, n}}\right]\right\}$ become a maximum $\frac{1}{4 i}$-separated subset of $\bar{\sigma}_{j}^{3}$. Retriangulate $\bar{\sigma}_{j}^{3}$ with these new vertices such that the mesh size of this new triangulation of $\bar{\sigma}_{j}^{3}$ is less than $\frac{1}{i}$.

We now choose a refinement $\tau_{i}$ of the initial triangulation $\tau_{0}$ so that the discrete subset $\cup_{k=1}^{3} \cup_{j}\left\{p_{k, j, h}\right\}_{h=1}^{m_{k, j}}$ become vertices with respect to $\tau_{i}$. Let $W(i, s)$ be the convex hull of $\cup_{k=1}^{3} \cup_{j}\left\{p_{k, j, h}\right\}_{h=1}^{m_{k, j}}$. Then $\partial W(i, s)$ is simplicial with respect to $\tau_{i}$. Hence, $W(i, s)$ is a convex PL-domain with the property $d_{H}\left(W(i, s), \Omega_{s}\right) \leq \frac{1}{i}$ and this finishes our proof.

\section{Gauss-Kronecker curvature measures on the convex part of a compact domain.}

In order to prove the isoperimetric inequality stated in the introduction as our Main Corollary we need to consider the inner Gauss-Kronecker curvature, and we shall derive the inequalities (5.2) below and (6.7) of Section 6 . The inequality (6.7) of the next section and standard arguments then imply the isoperimetric inequality.

Definition 5.1. Let $\Omega \subset X^{3}$ be a compact domain with non-empty interior. 
The total inner Gauss-Kronecker curvature measure of $\partial \Omega$ is defined by

$$
\int_{\partial \Omega} G K_{\partial \Omega}^{I} d A=\limsup _{\varepsilon \rightarrow 0} \int_{\partial \Omega_{-\varepsilon} \cap \partial \Omega_{-\varepsilon}^{*}} d\left(G K_{\partial \Omega_{-\varepsilon}^{*}}\right),
$$

where $\Omega_{-\varepsilon}=\{x \in \Omega \mid d(x, \partial \Omega) \geq \varepsilon\}$ and $\Omega_{-\varepsilon}^{*}$ is the convex hull of $\Omega_{-\varepsilon}$.

Let us make two elementary observations about $\partial W \cap\left(\partial W^{*}\right)$ for any compact set $W \subset X^{n}$, where $\partial W=\bar{W} \cap \overline{\left[X^{n}-W\right]}$.

Firstly we note that $\partial W \cap\left(\partial W^{*}\right) \neq \emptyset$ provided that $W \neq \emptyset$. In fact if $x_{0} \in X^{n}$ and $R=\max \left\{d\left(x, x_{0}\right) \mid x \in W\right\}>0$, then there exists $p \in$ $(\partial W) \cap \partial B_{R}\left(x_{0}\right)$. Since $W \subset B_{R}\left(x_{0}\right)$ and $B_{R}\left(x_{0}\right)$ is a convex set of $X^{n}$ we conclude that $W^{*} \subset B_{R}\left(x_{0}\right)$. Thus $p \in \partial W^{*}$ and hence $p \in \partial W \cap\left(\partial W^{*}\right)$.

Secondly, we observe that if $\Omega$ compact set with non-empty interior then $\Omega_{-\varepsilon}^{*} \neq \Omega$. To see this apply the previous discussion to the set $\Omega_{-\varepsilon}$ to conclude that there exists $p \in \partial \Omega_{-\varepsilon} \cap \partial \Omega_{-\varepsilon}^{*}$. Since $d(p, \partial \Omega) \geq d\left(\partial \Omega_{-\varepsilon}, \partial \Omega\right) \geq \varepsilon>0$ we find that $\partial \Omega_{-\varepsilon}^{*} \neq \partial \Omega$ and hence $\Omega_{-\varepsilon}^{*} \neq \Omega$, for any small $\varepsilon>0$.

Therefore the sets $\Omega_{-\varepsilon}^{*}$ have the properties that are convex sets with $\Omega_{-\varepsilon}^{*} \subset \Omega$ and $\Omega_{-\varepsilon}^{*} \neq \Omega$ which are fundamental for the arguments in Section 6.

We remark here that the right hand side of (5.1) is a finite number for a given compact convex domain, since we have the following observation.

Proposition 5.2. Let $\tau$ be a triangulation of $X^{3}$. Suppose that $\Omega$ is a convex and compact domain in $X^{3}$ and $m_{0}$ is the number of $n$-simplexes in $\operatorname{St}(\Omega)$. Then

$$
\int_{\partial \Omega} d\left(G K_{\partial \Omega}\right) \leq 4 \pi m_{0}
$$

Proof. Let $N_{0}=d(\Omega, \partial[\operatorname{St}(\Omega)])$. By Lemma 2.7 we know that for $s$ small, $\partial \Omega_{s}$ is transversal to $X^{(2)}$. Moreover

$$
\int_{\partial \Omega_{s}} d\left(G K_{\partial \Omega_{s}}\right) \underset{\text { def }}{=} \int_{\partial \Omega_{s}-\operatorname{Sing}\left(X^{3}\right)} d\left(G K_{\partial \Omega_{s}}\right) \leq \sum_{\sigma^{3} \subset \operatorname{St}(\Omega)} \int_{\bar{\sigma}^{3} \cap \partial \Omega_{s}} d\left(G K_{\partial \Omega_{s}}\right) .
$$

We can isometrically embed $\Omega_{s} \cap \bar{\sigma}^{3} \subset \bar{\sigma}^{3}$ into the Euclidean space $\mathbb{R}^{3}$ in order to estimate each of the integrals on the right hand side of the last inequality. Note that if $\Omega_{s}$ is convex, then $\Omega_{s} \cap \bar{\sigma}^{3}$ is also convex. Since $\left(\partial \Omega_{s}\right) \cap \bar{\sigma}^{3} \subset \partial\left(\Omega_{s} \cap \bar{\sigma}^{3}\right)$ we get that

$$
\int_{\bar{\sigma}^{3} \cap \partial \Omega_{s}} d\left(G K_{\partial \Omega_{s}}\right) \leq \int_{\partial\left[\Omega_{s} \cap \bar{\sigma}^{3}\right]} d\left(G K_{\partial\left[\Omega_{s} \cap \bar{\sigma}^{3}\right]}\right) .
$$


Furthermore, we find that

$$
\int_{\partial\left[\Omega_{s} \cap \bar{\sigma}^{3}\right]} d\left(G K_{\partial\left[\Omega_{s} \cap \bar{\sigma}^{3}\right]}\right)=4 \pi
$$

Using the inequalities above, we conclude that

$$
\int_{\partial \Omega_{s}} d\left(G K_{\partial \Omega_{s}}\right) \leq 4 \pi m_{0}
$$

where $m_{0}$ is the number of $n$-simplexes in $\operatorname{St}(\Omega)$.

In the rest of this subsection we will establish the inequality

$$
\int_{\partial \Omega \cap \partial \Omega^{*}} d\left(G K_{\partial \Omega^{*}}\right) \geq 4 \pi
$$

where $\Omega^{*}$ is the convex hull of $\Omega$ and $\Omega$ is compact domain. Observe that we cannot directly derive (5.2) from the Main Theorem, because it might happen that

$$
\int_{\partial \Omega^{*}-\partial \Omega} d\left(G K_{\partial \Omega^{*}}\right)>0
$$

For example, let $X^{2}$ be a cone of angle $4 \pi$ which can be constructed by taking four copies of Euclidean upper half planes $\mathbb{R}_{+}^{2}$, say $\left(\mathbb{R}_{+}^{2}\right)_{i}=\sigma_{i}^{2}$ for $i=1,2,3,4$ and gluing the half lines $[0,+\infty)$ of $\partial\left(\mathbb{R}_{+}^{2}\right)_{1}$ to the half lines $(-\infty, 0]$ of $\partial\left(\mathbb{R}_{+}^{2}\right)_{2}$, and so on. The resulting PL-surface $X^{2}$ satisfies the CAT(0) inequality. Let $\{(r, \theta) \mid r \geq 0,0 \leq \theta \leq 4 \pi\}$ be the polar coordinate system of $X^{2}$. If $\Omega=\{(r, \theta) \mid 1 \leq r \leq 2,0 \leq \theta \leq \pi\}$, then the origin $O \in\left[\partial \Omega^{*}-\partial \Omega\right]$ and the inequality (5.3) is satisfied.

To overcome this difficulty, we decompose the total curvature on $\partial \Omega^{*}$ into two parts:

$$
\int_{\partial \Omega^{*}} d\left(G K_{\partial \Omega^{*}}\right)=\int_{\partial \Omega^{*} \cap \partial \Omega} d\left(G K_{\partial \Omega^{*}}\right)+\int_{\partial \Omega^{*}-\partial \Omega} d\left(G K_{\partial \Omega^{*}}\right)
$$

Similarly, we decompose the non-negative error term as well:

$$
e_{3}\left(\Omega^{*}\right)=e_{3}\left(\Omega^{*}\right)\left|\Omega^{*} \cap \Omega+e_{3}\left(\Omega^{*}\right)\right|_{\Omega^{*}-\Omega}
$$

where for any $p \in[\partial \Omega] \cap \operatorname{Sing}\left(X^{3}\right)$

$$
\left.e_{3}\left(\Omega^{*}\right)\right|_{p}=\sum_{\sigma^{1} \subset S t(p)} \sum_{v \in \operatorname{Link}\left(p, \sigma^{1}\right)}\left[\left|\operatorname{Link}\left(\sigma^{1}, X^{3}\right)\right|-2 \pi\right] \sin \left[\theta_{p}^{*}\left(v, \Omega^{*}\right)\right]
$$


and for any subset $Q \subset \partial \Omega^{*}$

$$
\left.e_{3}(\Omega)\right|_{Q}=\left.\sum_{p \in Q \cap \operatorname{Sing}\left(X^{3}\right)} e_{3}(\Omega)\right|_{p} .
$$

The Main Theorem tells us that

$$
\int_{\partial \Omega^{*}} d\left(G K_{\partial \Omega^{*}}\right)=4 \pi+e_{3}\left(\Omega^{*}\right)=4 \pi+e_{3}\left(\Omega^{*}\right)\left|\Omega^{*} \cap \Omega+e_{3}\left(\Omega^{*}\right)\right|_{\Omega^{*}-\Omega}
$$

and $\left.e_{3}\left(\Omega^{*}\right)\right|_{p}$ is a non-negative function of $p$. If we can show that

$$
\int_{\partial \Omega^{*}-\partial \Omega} d\left(G K_{\partial \Omega^{*}}\right) \leq\left. e_{3}\left(\Omega^{*}\right)\right|_{\Omega^{*}-\Omega}
$$

then

$$
\int_{\partial \Omega^{*} \cap \partial \Omega} d\left(G K_{\partial \Omega^{*}}\right) \geq 4 \pi+e_{3}\left(\Omega^{*}\right) \mid \Omega^{*} \cap \Omega \geq 4 \pi
$$

and (5.2) follows immediately.

We remark that for any compact set $\Omega \subset \mathbb{R}^{n}$, Almgren observed that any point $p \in\left[\partial \Omega_{s}^{*}-\partial \Omega_{s}\right]$, there exists a straight line segment $\sigma_{p}$ passing through $p$ such that $\sigma_{p} \subset \partial \Omega_{s}^{*}$. Thus, $G K_{\partial \Omega_{s}^{*}}(p)=0$. Thus $\int_{\partial \Omega^{*}-\partial \Omega} G K_{\partial \Omega^{*}} d A=0$, and the inequality (5.4) holds trivially (cf. [Al2], page 455, line 1-4). Therefore inequality (5.4) can be viewed as an extension of Almgren's observation to PL manifolds.

In order to prove inequality (5.4) we need the following observation.

Proposition 5.3. Let $\Omega \subset X^{n}$ be a compact domain, $X^{n}$ be a simplyconnected PL-manifold of non-positive curvature and $p \in\left[\partial \Omega^{*}-\partial \Omega\right]$. Then there exists a geodesic line segment $\sigma:(-\epsilon, \epsilon) \rightarrow X^{n}$ such that $\sigma(0)=p$ and $\sigma \subset \Omega^{*}$, where $\Omega^{*}$ is the convex hull of $\Omega$. Consequently, $\operatorname{Diam}\left[\operatorname{Link}\left(p, \Omega^{*}\right)\right] \geq \pi$.

Proof. The existence of $\sigma$ follows from the definition of $\left[\partial \Omega^{*}-\partial \Omega\right]$. If $\sigma$ is a geodesic segment, then $\angle\left(\sigma_{\text {in }}^{\prime}(p), \sigma_{\text {out }}^{\prime}(p)\right) \geq \pi$ by Lemma 1.4. Thus, $\operatorname{Diam}\left[\operatorname{Link}\left(p, \Omega^{*}\right)\right] \geq \pi$ holds.

To verify inequality (5.4), it is sufficient to prove that for every $p \in$ $\left[\partial \Omega^{*}-\partial \Omega\right]$

$$
\left.G K_{\partial \Omega^{*}}\right|_{p} \leq\left. e_{3}\left(\Omega^{*}\right)\right|_{p} .
$$

Clearly, if $p \in\left[\partial \Omega^{*}-\partial \Omega\right]$ and if $p \notin \operatorname{Sing}\left(X^{3}\right)$, by Almgren's observation we still have $G K_{\partial \Omega^{*}}(p)=0$. Hence, (5.5) holds trivially in this case.

If $p \in \operatorname{Sing}\left(X^{3}\right) \cap\left[\partial \Omega^{*}-\partial \Omega\right]$ then we estimate both sides of (5.5) as follows. 
Proposition 5.4. Let $\Omega \subset X^{3}$ be a compact domain, $X^{3}$ be a simplyconnected PL-manifold of non-positive curvature and $p \in\left[\partial \Omega^{*}-\partial \Omega\right]$. Suppose that $A=\operatorname{Link}\left(p, \Omega^{*}\right)$ with $\operatorname{Diam}(A) \geq \pi$ and $A^{*}=\{v \in$ $\left.\operatorname{Link}\left(p, X^{3}\right) \mid d(v, A) \geq \frac{\pi}{2}\right\}$. In addition, suppose that

$$
\operatorname{Area}\left(A^{*}\right) \leq \sum_{v \in \operatorname{Sing}(L)}[|\operatorname{Link}(v, L)|-2 \pi] \sin \left[\theta_{A}^{*}(v)\right] .
$$

where $\theta_{A}^{*}(v)=\min \left\{d_{L}(v, A), \frac{\pi}{2}\right\}$. Then the inequalities (5.4)-(5.5) hold.

Proof. Observe that $\left.G K_{\partial \Omega^{*}}\right|_{p}=\operatorname{Area}\left(A^{*}\right)$. By Main Theorem, we know that

$$
\left.e_{3}\left(\Omega^{*}\right)\right|_{p}=\sum_{v \in \operatorname{Sing}(L)}[|\operatorname{Link}(v, L)|-2 \pi] \sin \left[\theta_{A}^{*}(v)\right]
$$

Thus, inequality (5.6) implies inequality (5.5) and inequality (5.4) as well.

Definition 5.5. Let $L$ be a piecewise spherical manifold satisfying the CAT(1) inequality.

(1) A closed subset $\Omega^{\prime} \subset L$ is said to be convex if for any pair of $\{v, w\} \subset \Omega^{\prime}$ with $d_{L}(v, w)<\pi$, the length minimizing spherical geodesic $\psi_{v, w}$ is contained in $\Omega^{\prime}$.

(2) A closed curve $\gamma$ is said to be convex to a domain $V$ if there is an $\epsilon$ such that for all $x, y \in \gamma$, with $d(x, y)<\epsilon$, the minimizing geodesic $\sigma_{x, y}$ from $x$ to $y$ satisfies $\sigma_{x, y} \subset \bar{V}$.

A family of convex subsets in $\operatorname{Link}\left(p, X^{n}\right)$ is given in the following proposition.

Proposition 5.6. Suppose that $\Omega$ is a domain with piecewise linear boundary and positive reach in $X^{n}$. Then, for any $p \in \partial \Omega, A=\operatorname{Link}(p, \Omega)$ is a convex subset. Moreover, the convex subset $A$ has positive reach $\geq \frac{\pi}{2}$ in $\operatorname{Link}\left(p, X^{n}\right)$.

Proof. This is a direct consequence of Proposition 1.6 and Proposition 1.7.

The following is a direct consequence of Proposition 4.2 and Propositions 5.3-5.4.

Corollary 5.7. Let $\Omega \subset X^{3}$ be a compact domain, $X^{3}$ be a simply-connected $P L$-manifold of non-positive curvature and $A \subset \operatorname{Link}\left(p, X^{3}\right)$ be a convex subset with $\operatorname{Diam}(A) \geq \pi$. Suppose that $A_{\frac{\pi}{2}}=\left\{v \in \operatorname{Link}\left(p, X^{3}\right) \mid d(v, A)<\right.$ $\left.\frac{\pi}{2}\right\}$ has the area

$$
\operatorname{Area}\left(A_{\frac{\pi}{2}}\right) \geq 4 \pi+\sum_{v \in \operatorname{Sing}(L)}[|\operatorname{Link}(v, L)|-2 \pi]\left\{1-\sin \left[\theta_{A}^{*}(v)\right]\right\} .
$$


where $\theta_{A}^{*}(v)=\min \left\{d_{L}(v, A), \frac{\pi}{2}\right\}$. Then the inequalities (5.4)-(5.5) hold.

The rest of this section is devoted to establish inequality (5.7) under the assumption $\operatorname{Diam}(A) \geq \pi$. Our proof of inequality (5.7) use the GaussBonnet formula and a new isoperimetric inequality (cf. Theorem 5.9 and Theorem 5.11 below) to estimate $\operatorname{Area}\left(A_{\frac{\pi}{2}}\right)$, where $A \subset L=\operatorname{Link}\left(p, X^{3}\right)$ is a convex subset.

Definition 5.8. (1) A domain $A$ in $L=\operatorname{Link}\left(p, X^{3}\right)$ is said to be piecewise spherical if its boundary $\partial A$ is a union of broken spherical geodesics.

(2) If $A \subset L$ is a convex domain, we define the length of its boundary to be $\ell(\partial A)=\lim _{s \rightarrow 0}\left|\partial A_{s}\right|$, where $A_{s}=\left\{w \in L \mid d_{L}(w, A)<s\right\}$ and $\left|\partial A_{s}\right|$ is the length of $\partial A_{s}$.

Recall that if $A \subset L$ is convex, $\left[\partial A_{s}-\operatorname{Sing}(L)\right]$ is a $C^{1,1}$ curve, and hence its length $\left|\partial A_{s}\right|$ is well-defined.

Note that any spherical geodesic $\sigma$ in $L$ gives rise to a convex subset $(\sigma)$ of $L$. Since $(\sigma)_{s}=\{v \in L \mid d(v, \sigma)<s\}$ it is easy to check that $\ell(\partial(\sigma))=$ $\lim _{s \rightarrow 0}\left|\partial(\sigma)_{s}\right|$ is equal to twice the length of $\sigma$.

Throughout the rest of this section we use $\partial A=\bar{A} \cap \overline{[L-A]}$ as a definition of $\partial A$, even if $\operatorname{dim} A \leq 1$.

Theorem 5.9. Let $A \subset L=\operatorname{Link}\left(p, X^{3}\right)$ be a simply-connected, compact, convex piecewise spherical domain. Then

$$
\operatorname{Area}\left(A_{\frac{\pi}{2}}\right)=\ell(\partial A)+2 \pi+\sum_{v \in \operatorname{Sing}(L)}[|\operatorname{Link}(v, L)|-2 \pi]\left\{1-\sin \left[\theta_{A}^{*}(v)\right]\right\} .
$$

where $\theta_{A}^{*}(v)=\min \left\{d_{L}(v, A), \frac{\pi}{2}\right\}$.

Proof. Let us consider the set $A_{\frac{\pi}{2}-\epsilon}$. Clearly, $\lim _{\epsilon \rightarrow 0} \operatorname{Area}\left[A_{\frac{\pi}{2}-\epsilon}\right]=$ $\operatorname{Area}\left(A_{\frac{\pi}{2}}\right)$. We use the Gauss-Bonnet formula to compute Area $\left[A_{\frac{\pi}{2}-\epsilon}\right]$. In order to do that we let $S^{\prime}=\left[A_{\frac{\pi}{2}}-A\right] \cap \operatorname{Sing}(L)=\left\{v_{1}, \ldots, v_{m}\right\}$, $S "=A \cap \operatorname{Sing}(L)=\left\{v_{m+1}, \ldots, v_{N}\right\}$ and let $\rho_{A}=\max _{1 \leq j \leq m}\left\{d_{L}\left(v_{j}, A\right)\right\}$ and $\epsilon_{A}=\min _{1 \leq j \leq m}\left\{d_{L}\left(v_{j}, A\right)\right\}$. Let $\epsilon$ sufficiently small so that $0<$ $\epsilon<\frac{1}{3} \min \left\{\frac{\pi}{2}-\rho_{A}, \epsilon_{A}\right\}$ and the disks $\left\{D_{\epsilon}\left(v_{i}\right)\right\}_{1 \leq i \leq N}$ are disjoint. Let $M_{\epsilon}^{2}=A_{\frac{\pi}{2}-\epsilon}-\left[\cup_{1 \leq i \leq N} D_{\epsilon}\left(v_{i}\right)\right]$. Clearly, $M_{\epsilon}^{2}$ is a smooth surface with a $C^{1,1}$ boundary. As before, we would like to compute the total geodesic curvature of $\partial M_{\epsilon}^{2}$. Let $\kappa_{\partial M_{\epsilon}^{2}}$ and $\kappa_{\partial\left[A_{\frac{\pi}{2}-\epsilon}\right]}$ be the geodesic curvature with respect to the inward unit normal vector.

A simple computation shows that

$$
\int_{\cup_{1 \leq i \leq N} \partial D_{\epsilon}\left(v_{i}\right)} \kappa_{\partial M_{\epsilon}^{2}} d \ell=\sum_{1 \leq i \leq N}\left|\operatorname{Link}\left(v_{i}, L\right)\right| .
$$


To calculate $\int_{\partial A_{\frac{\pi}{2}-\epsilon}} \kappa_{\partial M_{\epsilon}^{2}} d \ell$. We consider two sets in $\partial A_{\frac{\pi}{2}-\epsilon}$. Fix $t=t_{\epsilon}=$ $\frac{\pi}{2}-\epsilon$, clearly we have $\rho_{A}<t<\frac{\pi}{2}$. Thus, the closed curve $\partial A_{t}$ is a piecewise smooth curve. Furthermore, it is a $C^{1,1}$ curve. Since $A$ is a convex, $A$ has positive reach $\geq \frac{\pi}{2}$ by Proposition 5.6. Thus, there exists a nearest point projection $\pi_{A}: A_{\frac{\pi}{2}} \rightarrow A$. For any $p \in \partial A_{t}$ there is a unique length-minizing geodesic $\sigma_{p, \pi_{A}(p)}$ in $L$ from $\pi_{A}(p)$ to $p$. We consider the following subsets of $\partial A_{t}$ :

$$
\Psi_{t}=\left\{p \in \partial A_{t} \mid \sigma_{p, \pi_{A}(p)} \cap\left[A_{t}-A\right] \cap \operatorname{Sing}(L)=\emptyset\right\}
$$

and $\Phi_{t}=\left[\partial A_{t}\right]-\Psi_{t}$.

By our assumption, $\partial A$ is a broken spherical geodesic. We assume that there is a spherical triangulation $\tau$ of $L$ such that $\partial A$ becomes a spherical simplicial 1-dimensional complex, where each 1-simplex of $\tau$ is a geodesic segment of length $<\pi$ in $L$.

If $v_{j} \in(\partial A)^{(0)}$ is a vertex of $\partial A$, we let $\alpha_{j}=\left|\left[\operatorname{Link}\left(v_{j}, A\right)\right]^{*}\right|$ be the length of $\left[\operatorname{Link}\left(v_{j}, A\right)\right]^{*}$, where $\left[\operatorname{Link}\left(v_{j}, A\right)\right]^{*}$ is the dual link of $\operatorname{Link}\left(v_{j}, A\right)$ in $\operatorname{Link}\left(v_{j}, L\right)$. Using the polar coordinate system around the center $v_{j}$, one can show that

$$
\int_{\pi^{-1}\left(v_{j}\right) \cap \Psi_{t_{\epsilon}}} \kappa_{\partial M_{\epsilon}^{2}} d \ell=-\alpha_{j} \cos t_{\epsilon}
$$

If $\sigma_{i}^{1} \subset \partial A$ is an open 1-simplex of length $\ell_{i}$, using the Fermi coordinate system along the geodesic segment $\sigma_{i}^{1}$ one can derive

$$
\int_{\pi^{-1}\left(\sigma_{i}^{1}\right) \cap \Psi_{t_{\epsilon}}} \kappa_{\partial M_{\epsilon}^{2}} d \ell=\left[\sin t_{\epsilon}\right] \lim _{s \rightarrow 0}\left|\pi^{-1}\left(\sigma_{i}^{1}\right) \cap \partial A_{s}\right|
$$

Finally we consider the remaining $\int_{\pi^{-1}\left(\Phi_{t_{\epsilon}}\right)} \kappa_{\partial M_{\epsilon}^{2}} d \ell$. For any $0<s<\frac{\pi}{2}$, one can show that $A_{s}$ has positive reach $\geq \frac{\pi}{2}-s$ for $0 \leq s<\frac{\pi}{2}$. We let $\pi_{A_{s}}: A_{\frac{\pi}{2}} \rightarrow A_{s}$ be the nearest point projection from $A_{\frac{\pi}{2}}$ to $A_{s}$. Using the polar coordinate system around each singularity $v_{j} \in\left[A_{\frac{\pi}{2}}-A\right]$ and using the same reason as in the proof of (5.10), one can show that

$$
\left.\int_{\pi^{-1}\left(\Phi_{t_{\epsilon}}\right)} \kappa_{\partial M_{\epsilon}^{2}} d \ell=-\sum_{1 \leq j \leq m}\left[\left|\operatorname{Link}\left(v_{j}, L\right)\right|-2 \pi\right]\right]\left[\cos \left(t_{\epsilon}-\theta_{A}\left(v_{j}\right)\right)\right] .
$$

It follows from inequalities (5.9) to (5.12) that

$$
\begin{aligned}
& \int_{\partial M_{\epsilon}^{2}} \kappa_{\partial M_{\epsilon}^{2}}=\sum_{1 \leq i \leq N}\left|\operatorname{Link}\left(v_{i}, L\right)\right|+\sum_{\sigma_{i}^{1} \subset \partial A}\left(\sin t_{\epsilon}\right) \lim _{s \rightarrow 0}\left|\pi^{-1}\left(\sigma_{i}^{1}\right) \cap \partial A_{s}\right| \\
& -\sum_{\left.v_{i} \in \partial A\right)^{(0)}} \alpha_{i} \cos t_{\epsilon}-\sum_{1 \leq j \leq m}\left[\cos \left(t_{\epsilon}-\theta_{A}\left(v_{j}\right)\right)\right]\left[\left|\operatorname{Link}\left(v_{j}, L\right)\right|-2 \pi\right],
\end{aligned}
$$


where $t_{\epsilon}=\frac{\pi}{2}-\epsilon$. By the Gauss-Bonnet Theorem and letting $\epsilon \rightarrow 0$ in (4.13), we have that $t_{\epsilon}=\left[\frac{\pi}{2}-\epsilon\right] \rightarrow \frac{\pi}{2}$ and that

$$
\begin{aligned}
& \operatorname{Area}\left(A_{\frac{\pi}{2}}\right)=\lim _{\epsilon \rightarrow 0} \operatorname{Area}\left[M_{\epsilon}^{2}\right]=\lim _{\epsilon \rightarrow 0} \int_{M_{\epsilon}^{2}} K_{L} d A \\
= & 2 \pi(1-N)+\sum_{1 \leq i \leq N}\left|\operatorname{Link}\left(v_{i}, L\right)\right| \\
& +\ell(\partial A)+\sum_{1 \leq j \leq m}\left[\cos \left(\frac{\pi}{2}-\theta_{A}\left(v_{j}\right)\right)\right]\left[\left|\operatorname{Link}\left(v_{j}, L\right)\right|-2 \pi\right] \\
= & \ell(\partial A)+2 \pi+\sum_{v \in \operatorname{Sing}(L)}[|\operatorname{Link}(v, L)|-2 \pi]\left\{1-\sin \left[\theta_{A}^{*}(v)\right]\right\},
\end{aligned}
$$

where $K_{L}=1$ is the intrinsic curvature of $[L-\operatorname{Sing}(L)]$.

Using Lemma 2.5, we can extend the result of Theorem 5.9 to any compact convex domain.

Corollary 5.10. Let $A \subset L=\operatorname{Link}\left(p, X^{3}\right)$ be a simply-connected, compact, convex domain. Then

$$
\operatorname{Area}\left(A_{\frac{\pi}{2}}\right)=\ell(\partial A)+2 \pi+\sum_{v \in \operatorname{Sing}(L)}[|\operatorname{Link}(v, L)|-2 \pi]\left\{1-\sin \left[\theta_{A}^{*}(v)\right]\right\} .
$$

where $\theta_{A}^{*}(v)=\min \left\{d_{L}(v, A), \frac{\pi}{2}\right\}$.

Proof. For any given compact convex domain $A$, by taking a collection of of $i$ points on the boundary of $A$ and joining then by the minimizing geodesic one can construct a sequence of piecewise spherical convex domains $\{A(i)\}$ such that $A(i) \subset A$ and $\lim _{i \rightarrow \infty} d_{H}(A(i), A)=0, \lim _{i \rightarrow \infty} \operatorname{Area}\left([A(i)]_{\frac{\pi}{2}}\right)=$ $\operatorname{Area}\left(A_{\frac{\pi}{2}}\right), \lim _{i \rightarrow \infty} \ell[\partial A(i)]=\ell(\partial A)$, and $\lim _{i \rightarrow \infty} \sin \left[\theta_{A(i)}^{*}(v)\right]=\sin \left[\theta_{A}^{*}(v)\right]$ for any $v \in L$. Because (5.8) holds for each $A(i)$, by taking the limit, we conclude that (5.8) holds for any compact convex domain $A \subset L$.

In the next theorem we derive a new isoperimetric inequality for compact convex domains $A$ in $L=\operatorname{Link}\left(p, X^{3}\right)$.

Theorem 5.11. Let $A \subset L=\operatorname{Link}\left(p, X^{3}\right)$ be a compact convex domain. Suppose that $L$ satisfies the $C A T(1)$ equality. Then

$$
\ell(\partial A) \geq 2 \min \{\pi, \operatorname{Diam}(A)\},
$$

where $\operatorname{Diam}(A)$ denotes the diameter of $A$.

Our proof of Theorem 5.11 also show that for any compact convex domain $A \subset M^{2}$, where $M^{2}$ is a smooth Riemannian surface of curvature $0 \leq K_{M^{2}} \leq$ 
1, the isoperimetric inequality (5.15) holds. However the inequality (5.15) fails to hold if we replace $2 \min \{\pi, \operatorname{Diam}(A)\}$ by $2 \operatorname{Diam}(A)$. For example, let $M^{2}=\left\{(x, y, z) \in \mathbb{R}^{3} \mid x^{2}+y^{2}+\frac{z^{2}}{100}=1\right\}$ and $A=\left\{(x, y, z) \in M^{2} \mid z \geq\right.$ $0\}$. Then $\ell(\partial A)<2 \operatorname{Diam}(A)$. Similarly, one can construct an example of $A \subset L=\operatorname{Link}\left(p, X^{3}\right)$ such that $L$ satisfies the $C A T(1)$ equality and $A$ is convex, but $\ell(\partial A)<2 \operatorname{Diam}(A)$. In order to do that let $\mathcal{Y}=\left\{\left(x_{1}, x_{2}, x_{3}\right) \in\right.$ $\left.\mathbb{R}^{3} \mid \max \left\{\left|x_{1}\right|,\left|x_{2}\right|,\left|x_{3}\right|\right\}=\frac{\pi}{4}\right\}$. Thus, $\mathcal{Y}$ has six faces $\left\{F_{j}\right\}_{1 \leq j \leq 6}$. Each face $F_{j}$ is a square. The length of each side is $\frac{\pi}{2}$ and $\left|\partial F_{j}\right|=2 \pi$. Replace each face $F_{j}$ by a unit upper hemi-sphere $\Sigma_{j}=\left\{(x, y, z) \in \mathbb{R}^{3} \mid x^{2}+y^{2}+z^{2}=1, z \geq 0\right\}$ to get a new surface $L$. Clearly, $\left|\partial \Sigma_{j}\right|=\left|\partial F_{j}\right|=2 \pi$. The new resulting surface $L=\cup_{1 \leq j \leq 6} \Sigma_{j}$ is a piecewise spherical surface satisfying the $C A T(1)$ equality. Let $A=L=\cup_{1 \leq j \leq 5} \Sigma_{j}$. Then $A$ is a convex subset of $L$ and $\operatorname{Diam}(A)>\pi$. The boundary $\partial A=\partial \Sigma_{6}$ is a closed geodesic of length $2 \pi$. In this example, we have $\ell(\partial A)<2 \operatorname{Diam}(A)$, however inequality $(5.15)$ holds for $A$.

In order to prove Theorem 5.11 we need to recall some results.

When $L$ satisfies the $C A T(1)$ equality, a result of $[\mathrm{ChD}]$ implies that, $\operatorname{Inj}(L)$, the injectivity of $L$ is greater than or equal to $\pi$. The proof of Theorem 5.11 uses the so-called Birkhoff curve shortening process, which we briefly call it B.C.S.P, see [CC, p533-534] and [Cr2, p4]. This process depends on an integer $N>2$, where $N$ is chosen so large that $\ell(\gamma) / N$ is small than $\pi$. For a Lipschitz closed curve $\gamma$, the B.C.S.P. associates a new curve $\beta^{N}(\gamma)$ as well as a homotopy $\left\{\gamma_{s}\right\}_{0 \leq s \leq 1}$ from $\gamma=\gamma_{0}$ to $\beta^{N}(\gamma)=\gamma_{1}$. The homotopy $\left\{\gamma_{s}\right\}_{0 \leq s \leq 1}$ will be defined in such a way that $\ell\left(\gamma_{s_{1}}\right) \geq \ell\left(\gamma_{s_{2}}\right)$ whenever $s_{2} \geq s_{1}$.

Assume that $\gamma:[0,1] \rightarrow L$ is a closed curve parameterized proportional to arc-length; if not, the first part of the homotopy reparametrizes $\gamma$ so that it is. We then define $\gamma_{\frac{1}{2}}$ to be the unique piecewise geodesic closed curve such that $\gamma_{\frac{1}{2}}=\gamma\left(\frac{i}{N}\right)$ for all integers $i=1,2, \ldots, N$. For $s \in\left[0, \frac{1}{2}\right], \gamma_{s}$ will be given by

$$
\gamma_{s}\left(\frac{i}{N}+t\right)= \begin{cases}\tau_{i}^{s}(t), & 0 \leq t \leq \frac{2 s}{N}, \\ \gamma\left(\frac{i}{N}+t\right), & \frac{2 s}{N} \leq t \leq \frac{1}{N},\end{cases}
$$

where $\tau_{i}^{s}$ is the minimizing geodesic from $\gamma\left(\frac{i}{N}\right)$ to $\gamma\left(\frac{i}{N}+\frac{2 s}{N}\right)$ parameterized on the interval $\left[0, \frac{2 s}{N}\right]$ proportional to arc-length. Finally, $\gamma_{1}$ is defined as the unique closed geodesic with the shifted vertices: $\left\{\gamma_{1}\left(\frac{i}{N}+\frac{1}{2 N}\right)=\gamma_{\frac{1}{2}}\left(\frac{i}{N}+\right.\right.$ $\left.\left.\frac{1}{2 N}\right)\right\}_{0 \leq i \leq N-1}$, which is parameterized proportional to arc-length on each interval $\left[\frac{i}{N}+\frac{1}{2 N}, \frac{i+1}{N}+\frac{1}{2 N}\right]$. We then define $\gamma_{s}$ for $s \in\left[\frac{1}{2}, 1\right]$ to be the homotopy between $\gamma_{\frac{1}{2}}$ and $\gamma_{1}$ in the same way that $\gamma_{s}, s \in\left[0, \frac{1}{2}\right]$, homotopies 
from $\gamma_{0}$ to $\gamma_{\frac{1}{2}}$.

Lemma 5.12. ([Cr2]) Let $A \subset L=\operatorname{Link}\left(p, X^{3}\right)$ be a compact convex domain and let $\gamma$ be a parametrization of $\partial A$. Suppose that $L$ satisfies the $C A T(1)$ equality and $\ell(\gamma)=\ell$. Then if we apply B.C.S.P. with $N$ breaks to $\gamma$ the resulting curve must satisfy the following:

(1) $\gamma_{t} \subset A$;

(2) $\gamma_{t}$ is convex to $W_{t}=\overline{\left[A-\left\{x \in \gamma_{s} \mid 0 \leq s \leq t\right\}\right]} \cup \gamma_{t}$.

Proof. The proof is the same as that of Lemma 2.2 of [Cr2, page 7] with minor modifications. In [Cr2], the starting convex set $W_{0}$ was assumed to be a 2-dimensional open set. In our case, we use the compact convex sets $W_{t}$ above instead. However, the assumption, that the set $W_{0}$ is open, was not used for the proof of Lemma 2.2 of [Cr2]. Thus, the argument of [Cr2] remains to be valid for the proof of our Lemma 5.12.

Lemma 5.13. ([CC, p534]) Let $A \subset L=\operatorname{Link}\left(p, X^{3}\right)$ be a compact convex domain and let $\gamma$ be a parametrization of $\partial A$. Suppose that $L$ satisfies the $C A T(1)$ equality and $\ell(\gamma)=\ell$. Then either $A$ contains a non-trivial closed geodesic $\sigma$ of length $\ell(\sigma) \leq \ell$, or there exists a new homotopy $\varphi_{s}, s \in[0,1]$, which satisfies the following conditions:

(1) $\varphi_{1}=\gamma, \varphi_{0}=v_{0}$ is a point curve, $\ell\left(\varphi_{s}\right) \leq \ell$ for all $s ; \ell\left(\varphi_{s_{1}}\right) \leq \ell\left(\varphi_{s_{2}}\right)$ whenever $s_{1} \leq s_{2}$;

(2) $\varphi_{s}$ is convex to the domain $V_{s}=\left\{x \in \varphi_{t} \mid 0 \leq t \leq s\right\}$;

(3) $\left\{\varphi_{s}\right\}_{0 \leq s \leq 1}$ gives rise in a natural way to a map $F_{\varphi}$ from the two-disk $D^{2}$ into $A$ such that $F\left(\partial D^{2}\right)=\gamma$ and $F(0)=v_{0}$.

Proof. To prove Lemma 5.13 follow the argument in the proof of Corollary 1.3 of Part II of [CC] and use Lemma 5.12 instead of Lemma 1.2 of [Cr2].

In view of Lemma 5.13, we now prove Theorem 5.11.

Proof of Theorem 5.11. Let $\gamma:[0,1] \rightarrow \partial A$ be a parameterization of $\partial A$ with $\gamma(0)=\gamma(1)$. Because $L$ satisfies the $C A T(1)$ equality, by Lemma 1.2 of [ChD, p933], one knows that any non-trivial closed geodesic $\sigma$ has length $\ell(\sigma) \geq 2 \pi$. By Lemma 5.13 above, either $A$ contains a non-trivial closed geodesic $\sigma$ and hence

$$
\ell(\partial A)=\ell(\gamma)=\ell \geq \ell(\sigma) \geq 2 \pi
$$


or there exists a length non-decreasing family of closed curves $\left\{\varphi_{s}\right\}_{0 \leq s \leq 1}$ described as in Lemma 5.13 (1)-(3) above. Clearly, by our construction of $\varphi_{s}$ and $V_{s}$, we know that the function $h(s)=\operatorname{Diam}\left(V_{s}\right)$ is a non-decreasing continuous function of $s$. Since $V_{0}=\left\{v_{0}\right\}$ is a point, $h(0)=0$. By the intermediate value theorem, for each $t_{\epsilon}=\min \{\operatorname{Diam}(A), \pi\}-\epsilon$, there exists a domain $s(\epsilon) \in[0,1]$ such that $\operatorname{Diam}\left(V_{s(\epsilon)}\right)=t_{\epsilon}$. Recall that, by $[\mathrm{ChD}]$, the injectivity radius of $L$ is at least $\pi$. Since $0<t_{\epsilon}<\pi \leq \operatorname{Inj}(L)$, any geodesic segment $\sigma$ of length $\ell(\sigma)<\pi$ can be extended to a longer length minimizing geodesic segment $\tilde{\sigma}$ of length $\ell(\tilde{\sigma})=\ell(\sigma)+\epsilon_{1}$, as long as $0<\epsilon_{1}<\pi-\ell(\sigma)$. Therefore, the $\operatorname{Diam}\left(V_{s(\epsilon)}\right)$ must be achieved by a pair of boundary points in $V_{s(\epsilon)}$. Thus, there exists a geodesic segment $\Psi_{\epsilon}:[0,1] \rightarrow V_{s(\epsilon)}$ of $L$ with endpoints in $\partial V_{s(\epsilon)}$ and the length $\ell\left(\Psi_{\epsilon}\right)=\operatorname{Diam}\left(V_{s(\epsilon)}\right)$. The endpoints of $\Psi_{\epsilon}$ intersects with the boundary curve $\varphi_{s(\epsilon)}$ at two points, say $\varphi_{s(\epsilon)}(0)=$ $\varphi_{s(\epsilon)}(1)$ and $\varphi_{s(\epsilon)}\left(a_{\epsilon}\right)$ after reparametrization. Clearly, both path $\left.\varphi_{s(\epsilon)}\right|_{\left[0, a_{\epsilon}\right]}$ and path $\left.\varphi_{s(\epsilon)}\right|_{\left[a_{\epsilon}, 1\right]}$ have the length $\geq t_{\epsilon}$. Hence, we have $\ell\left(\varphi_{s(\epsilon)}\right) \geq 2 \ell\left(\Psi_{\epsilon}\right)=$ $2 \operatorname{Diam}\left(V_{s(\epsilon)}\right)=2 t_{\epsilon}=2[\min \{\operatorname{Diam}(A), \pi\}-\epsilon]$. This together with Lemma 5.13 (1) implies that

$$
\ell(\partial A)=\ell\left(\varphi_{1}\right) \geq \ell\left(\varphi_{s(\epsilon)}\right) \geq 2[\min \{\operatorname{Diam}(A), \pi\}-\epsilon]
$$

Letting $\epsilon \rightarrow 0$, we get $\ell(\partial A) \geq 2 \min \{\operatorname{Diam}(A), \pi\}$ for this case. Thus, we showed either $\ell(\partial A) \geq 2 \pi$ or $\ell(\partial A) \geq 2 \min \{\operatorname{Diam}(A), \pi\}$ holds.

The inequalities (5.3)-(5.7) now follow from Theorem 5.9 and Theorem 5.11 .

\section{Applications to a sharp isoperimetric inequality.}

In this section, we will prove the Main Corollary stated in Section $\S 0$. Our proof is similar to that of Kleiner in $[\mathrm{K}]$ for smooth manifolds. Additional efforts are needed, because our ambient PL-manifolds have singularities.

We first introduce the isoperimetric profile function. This function has been studied by several authors $[\mathrm{BBG}],[\mathrm{GLP}]$ and $[\mathrm{K}]$.

Definition 6.1. The isoperimetric profile function of a manifold $M, I_{M}$, is defined by

$$
\begin{gathered}
I_{M}(v)=\inf \{\text { Area }(\partial \Omega) \mid \Omega \subset M \text { is a compact domain with } \\
\text { rectifiable boundary } \partial \Omega, \operatorname{vol}(\Omega)=v\} .
\end{gathered}
$$

for any $v \in[0, \operatorname{vol}(M))$. 
Observe that the isoperimetric inequality (0.5) is equivalent to the inequality

$$
I_{X^{3}}(v) \geq I_{\mathbb{R}^{3}}(v) .
$$

for all $v \in(0, \infty)$. One difficulty in proving the inequality $(6.1)$ is that the space $X^{3}$ is not compact and therefore there is no guarantee that $I_{X^{3}}(v)$ is achieved by some compact domain $\Omega_{(v)}$. For this reason we consider an alternative isoperimetric profile function. For $x_{0} \in X^{3}$, we let $B_{r}\left(x_{0}\right)$ denote the closed metric ball centered at $x_{0}$ of radius $r$. Because $X^{3}=\bigcup_{r>0}^{\infty} B_{r}\left(x_{0}\right)$; it is sufficient to show that

$$
I_{B_{r}\left(x_{0}\right)}(v) \geq I_{\mathbb{R}^{3}}(v)
$$

for every $v \in\left[0, \operatorname{vol}_{3}\left(B_{r}\left(x_{0}\right)\right)\right)$ and every $r>0$. Let $X_{1}^{3}=B_{r}\left(x_{0}\right)$ for some $r>0$.

\section{6.a. The existence of optimal domains.}

Let $X_{1}^{n} \subset X^{n}$ be a compact, convex and simplicial domain. In this subsection we show the existence of minimizing domain for the isoperimetric profile function of $X_{1}^{n}$. We are not aware of a proof of the existence of those domains in spaces with singularities. Since the space $X^{n}$ can have singularities, we clarify this using some results from Geometric Measure Theory and other related fields, which are applicable to our PL-manifolds considered in our paper.

There are two main ingredients in our argument presented below. First, we observe that for each compact simplicial complex $X_{1}^{n}$, there is a simplicial embedding $F: X_{1}^{n} \rightarrow \mathbb{R}^{m}$ for sufficiently large $m$. Secondly, because $F$ is simplicial, $F$ and its inverse $F^{-1}$ must be Lipschitz maps between $X_{1}^{n}$ and $F\left(X_{1}^{n}\right)$. If a sequence of domains $\left\{\Omega_{j}\right\}_{j=1}^{+\infty}$ in $X_{1}^{n}$ satisfies

(1) Area $\left(\partial \Omega_{j}\right) \rightarrow I_{X_{1}^{n}}(v)$ where $I_{X_{1}^{n}}(v)=\inf \left\{\right.$ Area $(\partial \Omega) \mid \Omega \subset X_{1}^{n}$, $\operatorname{vol}(\Omega)=v, \partial \Omega$ is rectifiable $\}$;

(2) $\operatorname{vol}\left(\Omega_{j}\right) \equiv v$;

(3) $\Omega_{j}$ has piecewise smooth and rectifiable boundary;

then by a theorem of Federer-Fleming that $\left\{F\left(\Omega_{j}\right)\right\}_{j=1}^{+\infty}$ has a convergent subsequence which converges to a subset $Y_{v} \subset F\left(X_{1}^{n}\right) \subset \mathbb{R}^{m}$. Therefore, $\left\{\Omega_{j}\right\}_{j=1}^{+\infty}$ has a convergent subsequence which converges to an optimal domain $F^{-1}\left(Y_{v}\right)$. We now give the details of proof of the existence theorem. 
Since $X^{n}$ has non-positive curvature, the distance function is convex. Thus, the space $X^{n}$ is combable in the sense of Epstein, Thurston, et al., cf. [ET].

Proposition 6.2. Let $X_{1}^{n} \subset X^{n}$ be as above. Then there exists a constant number $b_{n}>0$ such that for any cellular $n$-chain $c$ in $X_{1}^{n}$

$$
\operatorname{vol}_{n}(c) \leq b_{n} \operatorname{diam}(\partial c) \operatorname{Area}(\partial c) \text {. }
$$

Proof. This is a direct consequence of Theorem 10.2.1 of [ET].

It is known that every compact simplicial complex $X_{1}^{n}$ of dimension $n$ can be simplicially embedded into the Euclidean space $\mathbb{R}^{m}$ of sufficiently higher dimension $m \gg n$ (after subdivision of $X_{1}^{n}$ if needed), see [Mun] p.13.

Definition 6.3. Let $U$ be an open set of $\mathbb{R}^{m}$ and consider the set of $n$-forms supported in $U, D^{n}(U)=\left\{\sum_{i_{1}<\ldots<i_{n}} a_{i_{1}, \ldots, i_{n}} d x^{i_{1}} \wedge \cdots \wedge d x^{i_{n}} \mid a_{i_{1} \cdots i_{n}} \in\right.$ $\left.C_{0}^{\infty}(U)\right\}$. An $n$-dimensional current (briefly called an $n$-current) in $U$ is a continuous linear functional on $D^{n}(U)$. The set of such $n$-currents will be denoted $D_{n}(U)$, see [Fed 2], [Sim]. If $w \in D^{n}(U)$, we denote the support of $w$ by $\operatorname{Spt}(w)$.

If $T$ is a current and $W$ is an open set $W \subset U$, then we define

$$
\sup _{\operatorname{Mass}_{W}(T)=} \sup _{|w| \leq 1, w \in D^{n}(U), \operatorname{Spt}(w) \subset W} T(w),
$$

and the $\mathrm{n}$-dimensional mass of a current $T$ is defined as

$$
\operatorname{Mass}_{n}(T)=\sup _{W \subset \mathbb{R}^{m}} \operatorname{Mass}_{W}(T)
$$

In this section, we are interested in integer multiplicity currents, see Definition 6.5 below.

Theorem 6.4. Let $X_{1}^{n} \hookrightarrow \mathbb{R}^{m}$ be as above. Suppose $T$ is a Lipschitz $(n-1)$ cycle in $X_{1}^{n}$. Then $T=Q+\partial R$, where $R$ is a Lipschitz $n$-chain, $Q$ is a simplicial $(n-1)$-cycle in $X_{1}^{n}$. Moreover,

$$
\left\{\begin{array}{l}
\operatorname{Mass}_{n-1}(Q) \leq b^{*} \operatorname{mass}_{n-1}(T) \\
\operatorname{Mass}_{n}(R) \leq b^{*} \operatorname{mass}_{n-1}(T)
\end{array}\right.
$$

where $Q$ and $R$ are contained in the smallest subcomplex of $X_{1}^{n}$ containing $T$ and $b^{*}>0$, depends only on $X_{1}^{n}$ and its triangulation.

Proof. This is a variant of a theorem of D. B. Epstein (cf. ([ET], Theorem 10.3.3, p.223-229). His proof is applicable to the PL-manifold $X_{1}^{n}$. 
Recall that any $n$-current $T$ can be viewed as a functional on the space of $n$-forms. By $T_{j} \rightarrow T$ in $U$, we mean that $\left\{T_{j}\right\}$ converges weakly to $T$ in the usual sense of distributions:

$$
T_{j} \rightarrow \mathrm{T} \text { in } \mathrm{U} \Leftrightarrow \lim _{j \rightarrow+\infty} T_{j}(w)=T(w), \forall w \in D^{n}(U) .
$$

Let $\mathcal{H}^{n}$ be $n$-dimensional Hausdorff measure on $\mathbb{R}^{m}$. Federer and Fleming $[\mathrm{FF}]$ introduced rectifiable and integer multiplicity currents.

Definition 6.5. If $T \in D_{n}(U)$ we say that $T$ is an integer multiplicity rectifiable $n$-current (briefly an integer multiplicity current) if it can be expressed

$$
T(\omega)=\int_{S}\langle\omega(x), \xi(x)\rangle \theta(x) d \mathcal{H}^{n}(x), \omega \in D^{n}(U),
$$

where $S$ is an $\mathcal{H}^{n}$-measurable countably $n$-rectifiable subset of $U, \theta$ is a locally $\mathcal{H}^{n}$-measure function such that for $\mathcal{H}^{n}$ - a.e. point $x \in S, \xi(x)$ can be expressed in the form $\tau_{1} \wedge \cdots \wedge \tau_{n}$, where $\tau_{1}, \ldots, \tau_{n}$ form an orthonormal basis for the approximate tangent space $T_{x} S$ (see [Sim, p.146]). Thus, $\xi$ orients the approximate tangent spaces of $S$ in an $\mathcal{H}^{n}$-measurable way. The function $\theta$ is called multiplicity and $\xi$ is called the orientation for $T$. We write $T=\tau(S, \xi, \theta)$.

An important theorem of Federer and Fleming $[\mathrm{FF}]$ tells us the following.

Theorem 6.6. If $\left\{T_{j}\right\} \subset D_{n}(U)$ is a sequence of integer multiplicity currents with

$$
\sup _{j \geq 1}\left[\operatorname{Mass}_{W}\left(T_{j}\right)+\operatorname{Mass}_{W}\left(\partial T_{j}\right)\right]<\infty, \forall W \subset \subset U
$$

then there is an integer multiplicity rectifiable $n$-current $T \in D_{n}(U)$ and a subsequence $\left\{T_{j^{\prime}}\right\}$ such that

$$
T_{j^{\prime}} \rightarrow T \text { in } U \text {. }
$$

Moreover, $T$ is contained in the smallest subsimplex of $U$ containing $\left\{T_{j}\right\}$.

Using Theorem 6.4, one can show that the subsequence $\left\{T_{j^{\prime}}\right\}$ converges to $T$ with respect to the flat metric topology. To describe the flat metric topology we let $U$ be an arbitrary open subset of $\mathbb{R}^{n+k}=\mathbb{R}^{m}$. Let $I$ be the subset $D_{n}(U)$ such that $\operatorname{Mass}_{W}(\partial T)<+\infty$ for all $W \subset \subset U$. On $I$ we define a family of pseudo-metrics $\left\{d_{W}\right\}_{W \subset \subset U}$ by

$$
\begin{array}{r}
d_{W}\left(T_{1}, T_{2}\right)=\inf \left\{\operatorname{Mass}_{W}(S)+\operatorname{Mass}_{W}(R) \mid T_{1}-T_{2}=\partial R+S\right. \\
\text { where } \left.R \in D_{n+1}(U), S \in D_{n}(U) \text { have integer multiplicity }\right\} .
\end{array}
$$


The following compactness theorem is essential to prove existence of minimal submanifolds.

Theorem 6.7. Let $T,\left\{T_{j}\right\} \subset D_{n}(U)$ be integer multiplicity rectifiable currents with $\sup _{j \geq 1}\left\{\operatorname{Mass}_{W}\left(T_{j}\right)+\operatorname{Mass}_{W}\left(\partial T_{j}\right)\right\}<\infty, \forall W \subset \subset U$. Then $T_{j} \rightarrow T$ in $U$ (in the sense of Theorem 6.6) if and only if $d_{W}\left(T_{j}, T\right) \rightarrow 0$ for each $W \subset \subset U$.

Proof. This is an analog of Theorem 31.2 of [Sim], p.180. The proof uses the deformation theorem (Theorem 6.4) and its direct consequences. We omit the details here.

We also need the following statement about the rectifiability of the limit currents.

Theorem 6.8. Suppose $\left\{T_{j}\right\} \subset D_{n}(U)$, suppose $T_{j}, \partial T_{j}$ are integer multiplicity for each $j$,

$$
\sup _{j \geq 1}\left\{\operatorname{Mass}_{W}\left(T_{j}\right)+\operatorname{Mass}_{W}\left(\partial T_{j}\right)\right\}<\infty, \forall W \subset \subset U,
$$

and suppose $T_{j} \rightarrow T \in D_{n}(U)$. Then $T$ is an integer multiplicity current and $T$ is rectifiable.

Proof. See [Sim, §25-27, §29-32].

The existence theorem of optimal domains is the following:

Theorem 6.9. Let $X_{1}^{n}$ be a convex, simplicial and compact subdomain of $X^{n}$. Then for each $v \in\left(0, \operatorname{vol}\left(X_{1}^{n}\right)\right)$ there exists a domain $\Omega_{(v)} \subset X_{1}^{n}$ with rectifiable boundary and

$$
\text { Area }\left(\partial \Omega_{(v)}\right)=\inf \left\{\operatorname{Area}(\partial \Omega) \mid \Omega \subset X_{1}^{n}, \Omega \text { is rectifiable and } \operatorname{vol}(\Omega)=v\right\} .
$$

Proof. Let $F: X_{1}^{n} \rightarrow \mathbb{R}^{m}$ be a simplicial embedding from $X_{1}^{n}$ into a higher dimensional Euclidean space $\mathbb{R}^{m}$. Then $F$ is a bi-Lipschitz homeomorphism between $X_{1}^{n}$ and its image $F\left(X_{1}^{n}\right)$, since $X^{n}$ is compact.

Choose a sequence of domains $\left\{\Omega_{j}\right\}_{j=1}^{+\infty}$ in $X_{1}^{n}$ such that (i) Area $\left(\partial \Omega_{j}\right) \rightarrow$ $I_{X_{1}^{n}}(v)$ where $I_{X_{1}^{n}}(v)=\inf \left\{\right.$ Area $(\partial \Omega) \mid \Omega \subset X_{1}^{n}, \operatorname{vol}(\Omega)=v, \partial \Omega$ is rectifiable\}; (ii) $\operatorname{vol}\left(\Omega_{j}\right) \equiv v$; and (iii) $\Omega_{j}$ has piecewise smooth and rectifiable boundary.

Let $T_{j}=F\left(\Omega_{j}\right)$. Because $F$ is a bi-Lipschitz homeomorphism (simplicial map), both $T_{j}$ and $\partial T_{j}$ are integer currents in $\mathbb{R}^{m}$. Let $U$ be a large ball containing $F\left(X^{n}\right)$ in $\mathbb{R}^{m}$. Then by the properties of $\left\{\Omega_{j}\right\}$, we know

$$
\sup _{j}\left\{\operatorname{Mass}_{W}\left(\partial T_{j}\right)+\operatorname{Mass}_{W}\left(T_{j}\right)\right\}<c<+\infty
$$


for some constant $c>0$ which is independent of $j$ and $W \subset \subset U$. It follows from Theorems 5.9-5.11 that there exists an integer current $T$ with multiplicity 1 and subsequence $\left\{T_{j_{i}}\right\}_{i=1}^{+\infty}$ such that $T_{j_{i}} \rightarrow T$ with respect to the flat metric topology. By Theorem 6.6, the limiting current $T$ is contained in the subsimplex $F\left(X_{1}^{n}\right)$ of $U$. Because $F$ is a bi-Lipschitz map from $X_{1}^{n}$ to $F\left(X_{1}^{n}\right)$, we conclude that

$$
\Omega_{j_{i}} \rightarrow F^{-1}(T)=\Omega_{(v)}
$$

and Area $\left(\partial \Omega_{(v)}\right)=\lim _{i \rightarrow+\infty}$ Area $\left(\Omega_{j_{i}}\right)=I_{X_{1}^{n}}(v)$. Further, we have vol $\left(\Omega_{(v)}\right)=$ $v$ and $\operatorname{Area}(\partial \Omega)=I_{X^{n}}(v)$. The domain $\Omega_{(v)}$ is the desired optimal domain.

\section{$6 . b$.
domains.}

6.b. Mean curvature and regularity of boundary for optimal

In this section, we assume that $X_{1}^{3} \subset X^{3}$ is a compact, convex and piecewise linear domain with non-empty interior, $\operatorname{Int}\left(X_{1}^{3}\right) \neq \emptyset$, and $\Omega_{(v)}$ is an optimal domain of volume $\operatorname{vol}_{3}\left(\Omega_{(v)}\right)=v$ and Area $\left(\partial \Omega_{(v)}\right)=I_{X_{1}^{3}}(v)$. In the above sub-section, we already discussed the existence of minimal domains with least boundary area and a given volume in a compact subcomplex $X_{1}^{3} \subset X^{3}$.

The purpose of this sub-section is to study mean curvature and regularity of boundary for optimal domains in PL-manifolds. Among other things, we show that the boundary of an optimal domain does not have any corner points (see Definition 6.11 below). Moreover, we shall show that if $X_{1}^{3}$ is a large geodesic ball, then the boundary of the optimal domain meets $\partial X_{1}^{3}$ tangentially at their intersection points, (cf. Corollary 6.16).

Proposition 6.10. Let $\Omega_{(v)} \subset X_{1}^{3} \subset X^{3}$ be as above and $x, y$, and $z$ be points where $\partial \Omega_{(v)}$ is twice differentiable. Assume that $x, y \in\left[\operatorname{Int}\left(X_{1}^{3}\right)-\right.$ $\left.\operatorname{Sing}\left(X^{3}\right)\right]$ and $z \in\left[\partial X_{1}^{3}-\operatorname{Sing}\left(X^{3}\right)\right]$. Then

$$
H(z) \leq H(x)=H(y)
$$

where $H$ denotes the mean curvature of $\partial \Omega_{(v)}$ with respect to the outward normal vector.

Proof. The proof is similar to that of Lemma 2.1 of [O] p1186-1187. Hence, we omit it here.

To study the regularity properties of optimal domains we need the definition of a corner point.

Definition 6.11. Let $v_{0} \in \operatorname{Link}\left(p, X^{3}\right)$, and let $U_{+}^{3}\left(p, v_{0}\right)=\mathcal{C}\left[B\left(v_{0}, \frac{\pi}{2}\right)\right]$ be the cone over $B\left(v_{0}, \frac{\pi}{2}\right)=\left\{v \in \operatorname{Link}\left(p, X^{3}\right) \mid d_{L}\left(v, v_{0}\right) \leq \frac{\pi}{2}\right\}$. Then $U_{+}^{3}\left(p, v_{0}\right)$ 
is called a half space in $T_{p}\left(X^{3}\right)$ in the direction $v_{0}$. Suppose that $\Omega \subset X^{3}$ is a compact domain with piecewise smooth boundary $\partial \Omega$. A point $p \in \partial \Omega$ is said to be a corner point if $T_{p}(\Omega)$ is contained in the interior of some half space $U_{+}^{3}$.

We have the following observation.

Proposition 6.12. Let $\Omega \subset X^{n}$ be as above with $n=3$. If there exists a corner point $p \in \partial \Omega$ then $\Omega$ cannot be an optimal domain.

Proof. The proof of Proposition 6.12 for the smooth case is along the line of that of Proposition 6.10, which is well-known. Such a proof can apply to our case as well.

If $\Omega_{(v)}$ is an optimal domain for the isoperimetric profile function $I_{X_{1}^{3}}$ then Proposition 6.12 tells us that for $p \in \partial \Omega_{(v)}$ cannot be a corner point; actually the tangent cone $T_{p}\left(\Omega_{(v)}\right)$ is area-minimizing.

Definition 6.13. A tangent cone $T_{p}(\partial \Omega)$ is said to be area-minimizing in $T_{p}\left(X^{3}\right)$ if, for any compact (2)-domain $\Sigma \subset T_{p}(\partial \Omega), \Sigma$ has the least (2)-dimensional measure among hypersurfaces $\Sigma^{\prime}$ in $T_{p}\left(X^{3}\right)$ with the same boundary $\partial \Sigma^{\prime}=\partial \Sigma$, i.e.,

$$
\text { Area }\left(\Sigma^{\prime}\right) \geq \operatorname{Area}(\Sigma)
$$

for any $\Sigma^{\prime} \subset T_{p}\left(X^{3}\right)$ with $\partial \Sigma^{\prime}=\partial \Sigma$ and $\Sigma \subset T_{p}(\partial \Omega)$.

The following fact is well-known and can be proved in the same way as in that of Proposition 6.10.

Corollary 6.14. Let $\Omega_{(v)} \subset X_{1}^{3}$ be an optimal domain with $\operatorname{vol}_{3}\left(\Omega_{(v)}\right)=v$ and Area $\left(\partial \Omega_{(v)}\right)=I_{X_{1}^{3}}(v)$. Then the tangent cone $T_{p}\left(\partial \Omega_{(v)}\right)$ is an areaminimizing cone in $T_{p}\left(X^{3}\right)$ for any $p \in \partial \Omega_{(v)}$.

The following result is an improvement of Proposition 6.12.

Theorem 6.15. Let $\Omega \subset X^{3}$ be a convex domain containing a point $p$. Suppose that $T_{p}(\partial \Omega)$ is an area-minimizing hypersurface in $T_{p}\left(X^{3}\right)$ and there exists $v_{0} \in \operatorname{Link}(p, \Omega)$ such that $\varangle\left(v_{0}, w\right) \leq \frac{\pi}{2}$ for all $w \in \operatorname{Link}(p, \Omega)$. Then $\varangle\left(v_{0}, w\right) \equiv \frac{\pi}{2}$ for all $w \in \operatorname{Link}(p, \partial \Omega)$.

Proof. By Corollary 6.14, we know that $T_{p}(\partial \Omega)$ is an area-minimizing hypersurface in $T_{p}\left(X^{3}\right)$. Suppose contrary, Theorem 6.15 were not true, we would obtain a contradiction as follows. There would be a $w_{0} \in \operatorname{Link}(p, \partial \Omega)$ with $\varangle\left(v_{0}, w_{0}\right)<\frac{\pi}{2}$. Let us now consider the hypersurface $T_{p}(\partial \Omega)$ around the line in the direction $w_{0}$, say $\ell_{w_{0}}$. Because $\varangle\left(v_{0}, w_{0}\right)<\frac{\pi}{2}$, using the proofs of Theorems 5.4.8-5.4.9 of Federer [Fe2, p629-630], one can show that $T_{p}(\partial \Omega)$ 
can not be area minimizing along $\ell_{w_{0}}$ in $T_{p}\left(X^{3}\right)$. This completes the proof of Theorem 6.15.

The following is a direct consequence of Theorem 6.15

Corollary 6.16. Let $\Omega_{(v)}$ be an optimal domain for the isoperimetric profile $I_{X_{1}^{3}}$ where $X_{1}^{3}=B_{r}\left(x_{0}\right)=\left\{x \in X^{3} \mid d\left(x, x_{0}\right) \leq r\right\}$, The boundary of the optimal domain $\Omega_{(v)}$, $\partial \Omega_{(v)}$ meets $\partial X_{1}^{3}$ tangentially.

Proof. For each $p \in \partial B_{r}\left(x_{0}\right)$ we observe that there is a geodesic $\varphi_{p x_{0}}$ from $p$ to $x_{0}$. Since $X^{3}$ has non-positive curvature, the law of cosine holds. Thus, for any $w \in T_{p}\left(B_{r}\left(x_{0}\right)\right)$ we have

$$
\varangle\left(w,\left(\varphi_{p x_{0}}\right)_{\text {out }}^{\prime}(p)\right) \leq \frac{\pi}{2} .
$$

Using Theorem 6.15 we obtain that

$$
\varangle\left(w,\left(\varphi_{p x_{0}}\right)_{\text {out }}^{\prime}(p)\right) \equiv \frac{\pi}{2}
$$

for every $w \in T_{p}\left(\partial \Omega\left(v_{0}\right)\right)$. Hence, the boundary $\partial \Omega(v)$ meets $\partial X_{1}^{3}=\partial B_{r}\left(x_{0}\right)$ tangentially at points in $\left[\partial \Omega(v) \cap \partial M_{1}^{3}\right]$.

\section{6.c. The proof of sharp isoperimetric comparison inequality.}

The isoperimetric profile function $I_{X_{1}^{3}}(v)$ of $X_{1}^{3}$ is a $C^{0, \alpha}-$ Hölder continuous function with exponent $\alpha=\frac{2}{3}$. For that reason we consider the derivative of the function $I_{X_{1}^{3}}(v)$ in the weak sense. We say $\left.\frac{d^{-} I_{X_{1}^{3}}}{d t}\right|_{t=v} \geq c$ if there exists a $C^{1}$ function $g$ defined on $[v-\varepsilon, v]$ with $g^{\prime}(v)=c, g(v)=I_{X_{1}^{3}}(v)$ and $g(t) \geq I_{X_{1}^{3}}(t)$ for $t \in[v-\varepsilon, v]$.

The mean curvature of $\partial \Omega_{v}$, where $\Omega_{(v)}$ is an optimal domain, is related to the left derivative of $I_{X_{1}^{3}}$ at $v$ as follows.

Proposition 6.17. Let $X_{1}^{3} \subset X^{3}, I_{X_{1}^{3}}$ and $\Omega_{v}$ be as above. Suppose that there is an open set $U \subset X^{3}-\operatorname{Sing}\left(X^{3}\right)$ such that $U \cap \partial \Omega_{v} \neq \emptyset$ is a $C^{2}$ hypersurface and $U \cap \partial \Omega_{v}$ has the constant mean curvature $H$ with respect to the outward normal vector field. Then

$$
\left.\frac{D^{-} I_{X_{1}^{3}}}{d t}\right|_{t=v} \geq H
$$

We now prove the first part of our Main Corollary. 
Proof of Main Corollary for the inequality part. Let $d=\operatorname{diam}(\Omega), x \in \Omega$ and $X_{1}^{3}=B_{4 d}(x)$ be the closed metric ball centered at $x$ of radius $4 d$.

In order to prove inequality (0.5) is enough to show that

$$
I_{X_{1}^{3}}(v) \geq I_{\mathbb{R}^{3}}(v),
$$

because if $\operatorname{vol}_{3}(\Omega)=v$, then we have that

$$
\operatorname{Area}(\partial \Omega) \geq I_{X_{1}^{3}}(v) \geq I_{\mathbb{R}^{3}}(v)=c_{3} v^{\frac{2}{3}}=c_{3}\left[\operatorname{vol}_{3}(\Omega)\right]^{\frac{2}{3}}
$$

and hence our theorem.

To prove the inequality (6.1) is enough to show that $I_{X_{1}^{3}}^{\frac{3}{2}}(v) \geq I_{\mathbb{R}^{3}}^{\frac{3}{2}}(v)$. Since $I_{X_{1}^{3}}(0)=I_{\mathbb{R}^{3}}(0)$ and the function $I_{X_{1}^{3}}$ is Hölder continuous with exponent $\frac{3}{2}$, it is sufficient to verify that

$$
\left.\left[I_{X_{1}^{3}}(v)\right]^{\frac{1}{2}} \frac{d^{-} I_{X_{1}^{3}}}{d t}\right|_{t=v} \geq\left.\left[I_{\mathbb{R}^{3}}(v)\right]^{\frac{1}{2}} \frac{d I_{\mathbb{R}^{3}}}{d t}\right|_{t=v}
$$

or equivalently,

$$
\left(\left.\frac{1}{2} \frac{d^{-} I_{X_{1}^{3}}}{d t}\right|_{t=v}\right)^{2} I_{X_{1}^{3}}(v) \geq\left(\left.\frac{1}{2} \frac{d I_{\mathbb{R}^{3}}}{d t}\right|_{t=v}\right)^{2} I_{\mathbb{R}^{3}}(v)
$$

where $\frac{d^{-} f}{d t}$ denotes the left weak derivative of $f$.

It is a classical result that the balls are the optimal domains in $\mathbb{R}^{3}$, then $I_{\mathbb{R}^{3}}(v)=c_{3} v^{\frac{2}{3}}$. Thus it suffices to show that

$$
\left(\left.\frac{1}{2} \frac{d^{-} I_{X_{1}^{3}}}{d t}\right|_{t=v}\right)^{2} I_{X_{1}^{3}}(v) \geq 4 \pi
$$

Let $\Omega_{(v)} \subset X_{1}^{3}$ be such that $\operatorname{vol}\left(\Omega_{(v)}\right)=v$ and Area $\left(\partial \Omega_{(v)}\right)=I_{X_{1}^{3}}(v)$. The existence of $\Omega_{(v)}$ is discussed in $\S 6$.a. If $v \leq v_{0}<\operatorname{vol}\left(X_{1}^{3}\right)$, then $\left[\operatorname{Int}\left(X_{1}^{3}\right)-\Omega_{(v)}\right] \neq \emptyset$ and $\partial \Omega_{(v)} \cap \operatorname{Int}\left(X^{3}\right) \neq \emptyset$. Because $\left[X_{1}^{3}-\operatorname{Sing}\left(X^{3}\right)\right]$ is open and dense in $\operatorname{Int}\left(X_{1}^{3}\right)$, the set $\partial \Omega_{(v)} \cap\left[X_{1}^{3}-\operatorname{Sing}\left(X^{3}\right)\right]$ is non-empty. The set of regular points of $\partial \Omega_{(v)}$ in $\left[\operatorname{Int}\left(X_{1}^{3}\right)-\operatorname{Sing}\left(X^{3}\right)\right]$ has positive measure. Let

$$
\Sigma_{1}=\left\{x \in \partial \Omega_{(v)} \mid x \in\left[\operatorname{Int}\left(X_{1}^{3}\right)-\operatorname{Sing}\left(X_{1}^{3}\right)\right], \partial \Omega_{(v)} \text { is regular at } x\right\} .
$$

Proposition 6.10 implies that $\Sigma_{1}$ has constant mean curvature $H_{\Sigma_{1}}$. Proposition 6.17 says that

$$
\left.\frac{d^{-} I_{X_{1}^{3}}}{d t}\right|_{t=v} \geq H_{\Sigma_{1}}
$$


Thus in order to verify the inequality (6.2) it is enough to show that

$$
\left[\frac{1}{2} H_{\Sigma_{1}}\right]^{2} \text { Area }\left(\partial \Omega_{(v)}\right) \geq 4 \pi
$$

Proposition 6.10 says that for an optimal domain $\Omega_{(v)}$

$$
H_{\partial \Omega_{(v)} \cap \partial X_{1}^{3}}(q) \leq H_{\Sigma_{1}}
$$

where $q$ is a regular point of $\left[\partial \Omega_{(v)}-\operatorname{Sing}\left(X^{3}\right)\right] \cap \partial X_{1}^{3}$, that is, $\partial \Omega_{(v)}$ is $C^{1,1}$ at $q$. Let us consider

$$
\Sigma_{2}=\left\{x \in \partial \Omega_{(v)} \mid x \notin \operatorname{Sing}\left(X^{3}\right), \partial \Omega_{(v)} \text { is regular at } x\right\} .
$$

Using the last inequality, we get

$$
\int_{\Sigma_{2}}\left[\frac{1}{2} H_{\Sigma_{1}}\right]^{2} d A \geq \int_{\Sigma_{2}}\left[\frac{1}{2} H_{\partial \Omega_{(v)}}\right]^{2} d A
$$

Therefore, the inequality (6.3) holds provided that

$$
\int_{\Sigma_{2}}\left[\frac{1}{2} H_{\partial \Omega_{(v)}}\right]^{2} d A \geq 4 \pi
$$

The convexity of $\partial X_{1}^{3}$ and inequality (6.4) imply that $H_{\partial \Omega_{(v)}}>0$. If $p \in$ $\partial \Omega_{v}^{*} \cap \Sigma_{2}$ then the principal curvatures of $\partial \Omega_{(v)}$ at $p$ are non-negative, hence

$$
\left[\frac{1}{2} H_{\partial \Omega_{(v)}}(p)\right]^{2} \geq \widetilde{G K}_{\partial \Omega_{(v)}}(p) .
$$

Thus, it is sufficient to show

$$
\int_{\Sigma_{2} \cap \partial \Omega_{(v)}^{*}} \widetilde{G K} \partial \Omega_{(v)}(p) d A \geq 4 \pi .
$$

There are two major ingredients in the proof of (6.6). We first show

$$
\int_{\left[\partial \Omega_{(v)}^{*} \cap \partial \Omega_{(v)}\right]-\operatorname{Sing}\left(X^{3}\right)} d\left(G K_{\partial \Omega_{(v)}}\right) \geq 4 \pi .
$$

Secondly, we prove in Claim 1 below that $\partial \Omega_{(v)}^{*} \cap \Sigma_{2}=\left[\partial \Omega_{(v)}^{*} \cap \partial \Omega_{(v)}\right]-$ $\operatorname{Sing}\left(X^{3}\right)$. The inequality (6.6) is a direct consequence of (6.7) and Claim 1 below. 
To show (6.7), we keep the notation as in Section 5. Recall that if $p \in\left[\partial \Omega_{(v)}^{*}\right] \cap \operatorname{Sing}\left(X^{3}\right)$,

$$
\left.e_{3}\left(\Omega_{(v)}^{*}\right)\right|_{p}=\sum_{\sigma^{1} \subset S t(p)} \sum_{v \in \operatorname{Link}\left(p, \sigma^{1}\right)}\left[\left|\operatorname{Link}\left(\sigma^{1}, X^{3}\right)\right|-2 \pi\right] \sin \left[\theta_{p}^{*}\left(v, \Omega_{(v)}^{*}\right)\right]
$$

and for any subset $Q \subset \partial \Omega_{(v)}^{*}$

$$
\left.e_{3}\left(\Omega_{(v)}^{*}\right)\right|_{Q}=\left.\sum_{p \in Q \cap \operatorname{Sing}\left(X^{3}\right)} e_{3}(\Omega)\right|_{p}
$$

By the results of Section 4 , if $\partial \Omega_{(v)}^{*}=V \cup W$ and if $V \cap W=\emptyset$, then

$$
\begin{aligned}
& \int_{V} d\left(G K_{\partial \Omega_{(v)}^{*}}\right)+\int_{W} d\left(G K_{\partial \Omega_{(v)}^{*}}\right) \\
& =\int_{\left[\partial \Omega_{(v)}^{*}\right]} d\left(G K_{\partial \Omega_{(v)}^{*}}\right) \\
& =4 \pi+\left.e_{3}\left(\Omega_{(v)}^{*}\right)\right|_{\partial \Omega_{(v)}^{*}} \\
& =4 \pi+\left.e_{3}\left(\Omega_{(v)}^{*}\right)\right|_{V}+\left.e_{3}\left(\Omega_{(v)}^{*}\right)\right|_{W}
\end{aligned}
$$

It follows that

$$
\begin{aligned}
& \int_{V} d\left(G K_{\partial \Omega_{(v)}^{*}}\right) \\
& =4 \pi+\left.e_{3}\left(\Omega_{(v)}^{*}\right)\right|_{V}+\left[\left.e_{3}\left(\Omega_{(v)}^{*}\right)\right|_{W}-\int_{W} d\left(G K_{\partial \Omega_{(v)}}\right)\right]
\end{aligned}
$$

We now choose $W=\left[\partial \Omega_{(v)}^{*}-\partial \Omega_{(v)}-\operatorname{Sing}\left(X^{3}\right)\right] \cup\left[\operatorname{Sing}\left(X^{3}\right) \cap\right.$ $\left.\partial \Omega_{(v)}^{*} \cap \partial \Omega_{(v)}\right]$. Since $\Omega_{(v)}$ is optimal, we showed in previous sub-section that $\partial \Omega_{(v)}$ does not have corner points, nor does $\partial \Omega_{(v)}^{*}$. In particular, $\operatorname{Diam}\left[\operatorname{Link}\left(p, \Omega^{*}\right)\right] \geq \pi$ for all $p \in \partial \Omega^{*}$. For each $p \in\left[\partial \Omega_{(v)}^{*}-\partial \Omega_{(v)}-\right.$ $\operatorname{Sing}\left(X^{3}\right)$ ], by Almgren's observation, we have

$$
\int_{\partial \Omega_{(v)}^{*}-\partial \Omega_{(v)}-\operatorname{Sing}\left(X^{3}\right)} \widetilde{G K}_{\partial \Omega_{(v)}^{*}} d A=0=\left.e_{3}\left(\Omega_{(v)}^{*}\right)\right|_{\partial \Omega_{(v)}^{*}-\partial \Omega_{(v)}-\operatorname{Sing}\left(X^{3}\right)} .
$$

For $p \in\left[\operatorname{Sing}\left(X^{3}\right) \cap \partial \Omega_{(v)}^{*} \cap \partial \Omega_{(v)}\right]$, we let $A=\operatorname{Link}\left(p, \Omega_{(v)}^{*}\right)$. By Proposition 4.2, Theorem 5.9 and Theorem 5.11 , because of $\operatorname{diam}(A) \geq \pi$ mentioned 
above, we have

$$
\begin{aligned}
& \int_{p} d\left(G K_{\left.\partial \Omega_{(v)}^{*}\right)}\right) \\
& =\operatorname{Area}\left(\operatorname{Link}\left(p, X^{3}\right)-\operatorname{Area}\left(A_{\frac{\pi}{2}}\right)\right. \\
& \leq \sum_{\sigma^{1} \subset S t(p)} \sum_{v \in \operatorname{Link}\left(p, \sigma^{1}\right)}\left[\left|\operatorname{Link}\left(\sigma^{1}, X^{3}\right)\right|-2 \pi\right] \sin \left[\theta_{p}^{*}\left(v, \Omega_{(v)}^{*}\right)\right] \\
& =\left.e_{3}\left(\Omega_{(v)}^{*}\right)\right|_{p} .
\end{aligned}
$$

It follows from (6.7.b) and (6.7.c) that

$$
\int_{W} d\left(G K_{\partial \Omega_{(v)}}\right) \leq\left. e_{3}\left(\Omega_{(v)}^{*}\right)\right|_{W}
$$

For $V=\partial \Omega_{(v)}^{*}-W=\left[\partial \Omega_{(v)}^{*} \cap \partial \Omega_{(v)}\right]-\operatorname{Sing}\left(X^{3}\right)$, by (6.7.a) and (6.7.d), we finally conclude that

$$
\begin{aligned}
& \int_{\left[\partial \Omega_{(v)}^{*} \cap \partial \Omega_{(v)}\right]-\operatorname{Sing}\left(X^{3}\right)} d\left(G K_{\left.\partial \Omega_{(v)}^{*}\right)}\right)=\int_{V} d\left(G K_{\partial \Omega_{(v)}}\right) \\
& =4 \pi+\left.e_{3}\left(\Omega_{(v)}^{*}\right)\right|_{V}+\left[\left.e_{3}\left(\Omega_{(v)}^{*}\right)\right|_{W}-\int_{\left[\partial \Omega_{(v)}^{*}\right] \cap W} d\left(G K_{\partial \Omega_{(v)}}\right)\right] \\
& \geq 4 \pi+\left.e_{3}\left(\Omega_{(v)}^{*}\right)\right|_{V} \geq 4 \pi .
\end{aligned}
$$

This finishes the proof of (6.7).

It remains to show

Claim 1: $\partial \Omega_{(v)}^{*} \cap \Sigma_{2}=\left[\partial \Omega_{(v)}^{*} \cap \partial \Omega_{(v)}\right]-\operatorname{Sing}\left(X^{3}\right)$.

It is clear that $\partial \Omega_{v}^{*} \cap \Sigma_{2} \subset\left\{\left[\partial \Omega_{v}^{*} \cap \partial \Omega_{(v)}\right]-\operatorname{Sing}\left(X^{3}\right)\right\}$. In order to prove that $\partial \Omega_{v}^{*} \cap \Sigma_{2} \supset\left\{\left[\partial \Omega_{v}^{*} \cap \partial \Omega_{(v)}\right]-\operatorname{Sing}\left(X^{3}\right)\right\}$, we write $\left[\partial \Omega_{v}^{*} \cap \partial \Omega_{(v)}-\right.$ $\left.\operatorname{Sing}\left(X^{3}\right)\right]=I \cup B$ where the set $I=\left\{\left[\partial \Omega_{v}^{*} \cap \partial \Omega_{(v)}-\operatorname{Sing}\left(X^{3}\right)\right] \cap \operatorname{Int}\left(X_{1}^{3}\right)\right\}$ and the set $B$ is defined as $B=\left\{\left[\partial \Omega_{v}^{*} \cap \partial \Omega_{(v)}-\operatorname{Sing}\left(X^{3}\right)\right] \cap \partial X_{1}^{3}\right\}$.

On the one hand we assert that $I$ is regular. Let $p \in I$. It is clear that $T_{p}\left(X^{3}\right)=\mathbb{R}^{3}$ and $S=T_{p}\left(\Omega_{(v)}\right)$ is a convex domain in $\mathbb{R}^{3}$. By Corollary 6.14 we see that $\partial S=T_{p}\left(\partial \Omega_{(v)}\right)$ is an area-minimizing hypersurface in $\mathbb{R}^{3}$. A result of Bombieri and Giusti says that if $\partial S$ is an area-minimizing (2)-dimensional current contained in a half space of $\mathbb{R}^{3}$, then $\partial S$ must be a hyperplane $\mathbb{R}^{2}$ in $\mathbb{R}^{3}$, see $[\mathrm{BG}, \mathrm{p} 42]$. This shows that $\partial \Omega_{(v)}$ must be differentiable at $p$. The regularity theory of minimal surfaces implies that $\partial \Omega_{(v)}$ is smooth and real analytic around $p$; hence $I$ is regular.

Furthermore, we assert that $B$ is regular. Let $p \in B$. Recall that $X_{1}^{3}=$ $B_{4 d}(x)$ is convex because the space $X^{3}$ has non-positive curvature . For each 
$p \in \partial X_{1}^{3}$, we let $\varphi_{p, x}$ be the geodesic from $p$ to $x$ and $v_{q}=\left(\varphi_{p x}\right)_{\text {out }}^{\prime}(p)$. Then $\varangle\left(w, v_{q}\right) \leq \frac{\pi}{2}$ for all $w \in \operatorname{Link}\left(p, X_{1}^{3}\right)$. If $\partial \Omega_{(v)}$ makes an angle with $\partial X_{1}^{3}$ less than $\pi$ at $p$, then $p$ is a corner point of $\partial \Omega_{(v)}$ and Theorem 6.15 says that $\Omega$ is not an optimal domain. Therefore, $\partial \Omega_{(v)}$ touches $\partial X_{1}^{3}$ tangentially at points $p \in \partial \Omega_{(v)} \cap \partial X_{1}^{3}$. This shows that $\partial \Omega$ is $C^{1,1}$ at $p \in\left[\partial \Omega_{(v)}-\operatorname{Sing}\left(X^{3}\right)\right] \cap \partial X_{1}^{3}$. Thus $B$ is regular and this completes the proof of Claim 1 .

We now observe that, since $\Sigma_{2}$ is regular,

$$
\left.G K_{\partial \Omega_{(v)}^{*}}\right|_{p}=\left.\widetilde{G K}_{\partial \Omega_{(v)}}\right|_{p}
$$

for all $p \in \Sigma_{2}$. The inequality (6.6) now follows from (6.7)-(6.8) and Claim 1. This completes the proof of the inequality (0.5), i.e., the inequality part of Main Corollary.

It is natural to ask for which domains $\Omega$ the equality holds in the inequality (0.5). The answer to this question is given in our Main Corollary.

Proof of Main Corollary for the equality case. Suppose now there exists a compact domain $\Omega_{(v)}$ in $X^{3}$ such that

$$
\operatorname{Area}\left(\partial \Omega_{(v)}\right)=c_{3}\left[\operatorname{vol}_{3}\left(\Omega_{(v)}\right)\right]^{\frac{2}{3}} .
$$

Then the inequalities in (6.5) and (6.6) become equalities. In this case, $\Omega_{(v)}$ has the following extra properties:

(i) $\Omega_{(v)}$ is a convex domain and $\partial \Omega_{(v)}$ is regular at $p \in \partial \Omega_{(v)}-\operatorname{Sing}\left(X^{3}\right)$.

(ii) Almost all points of $\partial \Omega_{(v)}$ are umbilical points with respect to inner unit normal vector; (this is because $\frac{1}{2}\left[H_{\partial \Omega_{(v)}}(p)\right]^{2}=\widetilde{G K}_{\partial \Omega_{(v)}}(p)$ holds almost everywhere);

(iii) If $r_{0}=\left[\frac{\operatorname{Area}\left(\partial \Omega_{(v)}\right)}{4 \pi}\right]^{\frac{1}{2}}$, then the equalities

$$
\begin{aligned}
& \int_{\partial \Omega_{(v)}^{*}} d\left(G K_{\partial \Omega_{(v)}}\right)=\int_{\Sigma_{2} \cap \partial \Omega_{(v)}^{*}} \widetilde{G K_{\partial \Omega}}(p) d A \\
& =\int_{\Sigma_{2} \cap \partial \Omega_{(v)}^{*}}\left[\frac{1}{2} H_{\partial \Omega_{(v)}}(p)\right]^{2} d A=4 \pi
\end{aligned}
$$

imply that $\Sigma_{2}$ has full measure in $\partial \Omega_{(v)}^{*}$. Furthermore, almost all points in $\partial \Omega_{(v)}$ have the same inner principal curvature $-\frac{1}{r_{0}}$ with respect to the inward unit normal vector.

It is well-known that an umbilical hypersurface $\Sigma^{2}$ with principal curvatures equal to $\pm \frac{1}{r_{0}}$ in the Euclidean space $\mathbb{R}^{3}$ must be isometric to a piece of the Euclidean round sphere of radius $r_{0}$. For each Euclidean $n$-simplex $\sigma_{j}^{3}$ 
with $\operatorname{Int}\left(\sigma_{j}^{3}\right) \cap \partial \Omega_{(v)} \neq \emptyset$, we know that $\Sigma_{j}=\sigma_{j}^{3} \cap \partial \Omega_{(v)}$ is a hypersurface of almost all umbilical points. The regularity theory for elliptic equations imply that $\Sigma_{j}$ is a smooth hypersurface. Therefore, $\partial \Omega_{(v)}=\bigcup_{j} \Sigma_{j}$ is a piecewise spherical hypersurface of curvature $K=\frac{1}{r_{0}^{2}}$, with only possible singularities when it meets $\operatorname{Sing}\left(X^{3}\right)$. Furthermore, by the minimizing property of $\partial \Omega_{(v)}=\partial \Omega_{(v)}^{*}$, the discussion in $\S 6$.b implies that $\operatorname{Diam}\left[\operatorname{Link}\left(p, \Omega_{(v)}\right)\right] \geq \pi$ and the total length of $\operatorname{Link}\left(p, \partial \Omega_{(v)}\right)$ satisfies $\left|\operatorname{Link}\left(p, \partial \Omega_{(v)}\right)\right| \geq 2 \pi$. A theorem of Gromov says that if $\partial \Omega_{(v)}$ is a piecewise spherical space of constant curvature $K=\frac{1}{r_{0}^{2}}$ and if $\operatorname{Link}\left(p, \partial \Omega_{(v)}\right)$ satisfies the CAT(1) for each $p \in \partial \Omega_{(v)}$, then $\partial \Omega_{(v)}$ satisfies the $\operatorname{CAT}\left(\frac{1}{r_{0}^{2}}\right)$ inequality, see $[\mathrm{ChD}]$. Theorem 1.2 and its proof imply that

$$
\operatorname{Area}\left(\partial \Omega_{(v)}\right) \geq \operatorname{Area}\left(S^{2}\left(r_{0}\right)\right)
$$

where $S^{2}\left(r_{0}\right)$ denotes the round sphere of radius $r_{0}$ in $\mathbb{R}^{3}$ and equality holds in (6.9) if and only if $\partial \Omega_{(v)}$ is isometric to $S^{2}\left(r_{0}\right)$.

On the other hand, equalities in (0.5) and its proof tell us that

$$
\begin{aligned}
4 \pi & =\int_{\partial \Omega_{(v)}} G K^{I} d A=\int_{\partial \Omega_{(v)}-\operatorname{Sing}\left(X^{3}\right)} \frac{1}{r_{0}^{2}} d A \\
& =\operatorname{Area}\left(\partial \Omega_{(v)}\right) \frac{1}{r_{0}^{2}} .
\end{aligned}
$$

Thus Area $\left(\partial \Omega_{(v)}\right)=\operatorname{Area}\left(S^{2}\left(r_{0}\right)\right)$ and $\partial \Omega_{(v)}$ is isometric to $S^{2}\left(r_{0}\right)$.

In order to show that $\Omega_{(v)}$ is isometric to an Euclidean ball $B_{r_{0}}(0)$ of radius $r_{0}$, we glue $\Omega_{(v)}$ into $\left[\mathbb{R}^{3}-B_{r_{0}}(0)\right]$ along $\partial \Omega_{(v)} \cong S^{2}\left(r_{0}\right)=\partial B_{r_{0}}(0)$, getting a new $\operatorname{CAT}(0)$-space $\widehat{X}^{3}$.

We shall show that $\Omega_{(v)}$ has no interior singularities. We also observe that $\partial\left(\Omega_{(v)}\right)_{s} \subset \mathbb{R}^{3}-B_{r_{0}}(0) \subset \widehat{X}^{3}$ is isometric to $S^{2}\left(r_{0}+s\right)=\partial B_{r_{0}+s}(0)$ in $\mathbb{R}^{3}$. By Theorem 1.2, it is sufficient to show that Area $\left(\operatorname{Link}\left(x, \widehat{X}^{3}\right)\right) \leq 4 \pi$, for $x \in \bar{\Omega}_{(v)}$. Since $\partial\left(\Omega_{(v)}\right)_{s}$ is isometric to $S^{2}\left(r_{0}+s\right)$ in $\mathbb{R}^{3}-B_{r_{0}}(0)$, we have

$$
\int_{\partial\left(\Omega_{(v)}\right)_{s}} G K_{\partial\left(\Omega_{(v)}\right)_{s}} d A=4 \pi
$$

For any given $x_{0} \in \bar{\Omega}_{(v)}$, we define a map $F: \partial\left(\Omega_{(v)}\right)_{s} \rightarrow \operatorname{Link}\left(x_{0}, \widehat{X}^{3}\right)$ by $F(q)=\left(\varphi_{q}\right)_{\text {out }}^{\prime}\left(x_{0}\right)$ where $\varphi_{q}:[0,+\infty) \rightarrow \widehat{X}^{3}$ is a geodesic ray asymptotic to the geodesic ray $\psi_{q}:[0,+\infty) \rightarrow\left[\mathbb{R}^{3}-B_{r_{0}}(0)\right] \hookrightarrow \widehat{X}^{3}$ given by $\psi_{q}(t)=$ 
$q+t N(q)$, where $N(q)$ is the outward unit normal vector of $\partial\left(\Omega_{(v)}\right)_{s}=$ $\partial B_{r_{0}+s}(0)$.

If $p, q \in \partial\left(\Omega_{(v)}\right)_{s}=\partial B_{r_{0}+s}(0)$ with $d(p, q)<\frac{\varepsilon}{2}$, the geodesic segment $\eta_{p, q}$ from $p$ to $q$ lies entirely in $\widehat{X}^{3}-\Omega_{(v)}=\mathbb{R}^{3}-B_{r_{0}}(0)$. Let $\mathbb{P}_{p}^{q}$ be the parallel translation from $p$ to $q$ along $\eta_{p, q}$ in $\mathbb{R}^{3}-B_{r_{0}}(0)$ and define $\varangle(N(p), N(q))$ def $\varangle\left(N(q), \mathbb{P}_{p}^{q} N(p)\right)$. It is easy to check that $\varangle(F(p), F(q)) \leq \varangle(N(p), N(q))$, as long as $d(p, q)<\frac{s}{2}, p, q \in \partial B_{r_{0}+s}(0)=\partial\left(\Omega_{(v)}\right)_{s}$. It follows from the last inequality that

$$
\operatorname{Area}\left(\operatorname{Link}\left(x_{0}, \widehat{X}\right)\right) \leq \int_{\partial\left(\Omega_{(v)}\right)_{s}} G K_{\partial\left(\Omega_{(v)}\right)_{s}} d A=4 \pi .
$$

This together with Theorem 1.2 imply that Link $\left(x_{0}, \widehat{X}\right)$ is isometric to $S^{2}(1)$. Thus $\widehat{X}$ is smooth at all $x_{0} \in \bar{\Omega}_{(v)}$ and $\bar{\Omega}_{(v)}$ has no singularities. Finally, we apply Theorem 7 of $[\mathrm{SZ}]$ to $\widehat{X}^{3}$ and conclude that $\widehat{X}^{3}$ is isometric to $\mathbb{R}^{3}$ and $\Omega_{(v)}$ is isometric to the round Euclidean ball $B_{r_{0}}(0)$ of radius $r_{0}$.

\section{References.}

[ABN] Aleksandrov, A. D., Berestovskii, V. N. and Nikolaev, I. G., Generalized Riemannian spaces, Russian Math. Surveys vol 41:3 (1986), $3-44$.

[Al1] Almgren, F., Optimal Isoperimetric Inequalities, Bull. Amer. Math. Soc. vol 13, No.2 (1985), 123-127.

[Al2] Optimal Isoperimetric Inequalities, Indiana University Math. Jour. vol 35, No. 3 (1986), 451-547.

[BB] Ballmann, W. and Buyalo, S., Nonpositively curved metrics on 2polyhedra, Math. Z. vol 222, no.1 (1996), 97-134.

[BBG] Berard P., Besson G., and Gallot S., Sur une inégelité isopérimetrique qui généralize celle de Paul-Levy-Gromov, Invent. Math. vol $\mathbf{8 0}$ (1985), 295-308.

[BG] Bombieri, E. and Giusti, E., Harnack's inequality for elliptic differential equations on minimal surfaces, Inventiones Math. vol 15 (1972), 24-46. 
[Bri] Bridson, M., Geodesics and curvature in metric simplicial complexes, in book "Group Theory from a Geometrical Viewpoint" (1991), World Scientific, Singapore, New Jersey, 373-463.

[BH] Bridson, M. and Haefliger, A., Metric Spaces of Non-positive Curvature, Springer-Verlag Berlin Heidelberg 1999.

[CC] Calabi, E. and Cao, J., Simple closed geodesics on convex surfaces, J. Diff. Geom. vol 36 (1992), 517-549.

[ChD] Charney, R. and Davis, M., Singular metrics of non-positive curvature on branched covers of Riemannian manifolds, American Journal of Math. vol. 115 (1993), 929-1009.

[CMS] Cheeger, J. Muller, W. and Schrader, R., On the Curvature of Piecewise Flat Spaces, Commun. Math. Phys. vol 92 (1984), 405-454.

[CL] Chern, S. S. and Lashof, R., On the total curvature of immersed manifolds, Amer. J. Math. vol. 79 (1957), 306-318.

[Cr1] Croke C., A sharp four dimensional isoperimetric inequality, Comm. Math. Helv. vol 59 (1984), 419-435.

[Cr2] Area and the length of the shortest closed geodesic, J. Diff. Geom. vol 27 (1988), 1-21.

[ET] Epstein, D. B., Cannon, J., Holt, D., Levy, S., Paterson, M. and Thurston, W., Word Processing in Groups, Bartlett and Jones, Boston, 1992.

[Fe1] Federer, H., Curvature measure, Trans. Amer. Math. Soc. vol 93 (1959), 418-491.

[Fe2] Geometric Measure Theory, Springer-Verlag, BerlinNew York, 1969.

[FF] Federer, H. and Fleming, W., Normal and integral currents, Annals of Math. vol 72 (1960), 458-520.

[GLP] Gromov, M., Lafontaine, J., Pansu, P., Structures métriques pour les variétés Riemanniennes, Cedic/Fernand Nathan, Paris, 1981.

[K] Kleiner, B., An Isoperimetric Comparison Theorem, Inventiones Mathematicae vol 108 (1992), 37-47. 
[Mun] Munkres, J., Elements of Algebraic Topology, Addison-Wesley Publishing Company, 1984.

[O] Osserman, R., The Isoperimetric Inequality, Bulletin of the American Math. Soc. vol 84 (1978), 1182-1238.

[SY] Schoen, Y. and Yau, S. T., Lectures on Differential Geometry, International Press, Boston, 1994.

[SZ] Schroeder, V. and Ziller, W., Local rigidity of symmetric spaces, Trans. Amer. Math. Soc. vol 320 (1990), 145-160.

[Sim] Simon, L., Lectures on Geometric Measure Theory, Proceedings of the Center for Mathematical Analysis, Australian National University, Volume 3, 1983, first published in Australia, 1984.

[W] Weil, A., Sur les surfaces on curboure negative, C. R. Acad. Sci., Paris vol 182 (1926), 1069-1071.

JiANGUO CAO

Department of Mathematics

University of Notre Dame

Notre Dame, IN 46556, USA

cao.7@nd.edu

José F. EsCOBAR

Department of Mathematics

CORNELl UNIVERSITY

ITHACA, NY 14853, USA

escobar@math . cornell. edu

Received March 7, 2000. 